\title{
AFFINE GRASSMANNIANS AND THE GEOMETRIC SATAKE IN MIXED CHARACTERISTIC
}

\author{
XINWEN ZHU
}

\begin{abstract}
We endow the set of lattices in $\mathbb{Q}_{p}^{n}$ with a reasonable algebro-geometric structure. As a result, we prove the representability of affine Grassmannians and establish the geometric Satake correspondence in mixed characteristic. We also give an application of our theory to the study of Rapoport-Zink spaces.
\end{abstract}

\section{Contents}

Introduction

0.1. Mixed characteristic affine Grassmannian

0.2. The geometric Satake

0.3. Dimension of affine Deligne-Lusztig varieties

0.4. Organization

0.5. Notations

0.6. Acknowledgement

1. Affine Grassmannians

1.1. $p$-adic loop groups

1.2. The affine Grassmannian for $\mathrm{GL}_{n}$

1.3. Representability

1.4. "Demazure resolution"

1.5. The affine Grassmannian and affine flag variety

2. The geometric Satake

2.1. The category $\mathrm{P}_{L^{+} G}\left(\mathrm{Gr}_{G}\right)$

2.2. Semi-infinite orbits

2.3. Monoidal structure on $\mathrm{H}^{*}$

2.4. The commutativity constraints

2.5. Conclusion

3. Dimension of affine Deligne-Lusztig varieties

3.1. Dimension of affine Deligne-Lusztig varieties

3.2. Affine Deligne-Lusztig varieties and Rapoport-Zink spaces

Appendix A. Generalities on perfect schemes

A.1. Perfect schemes and perfect algebraic spaces

A.2. Perfect algebraic spaces perfectly of finite presentation

A.3. $\ell$-adic sheaves

Appendix B. More on mixed characteristic affine Grassmannians

B.1. The determinant line bundle

B.2. Deperfection

B.3. Example: $\widetilde{\mathrm{Gr}}_{2} \rightarrow \overline{\mathrm{Gr}}_{2}$.

References 


\section{INTRODUCTION}

\subsection{Mixed characteristic affine Grassmannian.}

0.1.1. Let $F$ be a non-archimedean local field, i.e., $F$ is either $\mathbb{F}_{q}((t))$ or a finite extension of $\mathbb{Q}_{p}$. Let $\mathcal{O} \subset F$ be its ring of integers, and $k$ be its residue field. Let $V=F^{n}$ be an $n$-dimensional $F$-vector space. A lattice of $V$ is a finitely generated $\mathcal{O}$-submodule $\Lambda$ of $V$ such that $\Lambda \otimes F=V$. For example, $\Lambda_{0}=\mathcal{O}^{n}$ is a lattice in $V$ (usually called the standard lattice). Note that every other lattice in $V$ can be obtained from $\Lambda_{0}$ by a linear transformation $g: V \rightarrow V$, and therefore, the set of lattices in $V$ is identified with the set $\operatorname{Aut}(V) / \operatorname{Aut}\left(\Lambda_{0}\right)$, or $\mathrm{GL}_{n}(F) / \mathrm{GL}_{n}(\mathcal{O})$ if we use the standard basis.

For various applications in number theory, representation theory and algebraic geometry, it is highly desirable to realize this set as the set of $(k$-)points of some (reasonable, infinite dimensional) algebraic variety, defined over $k$. If $F=k((t))$, such algebro-geometric object, called the affine Grassmannian, does exist, and plays a fundamental role in geometric representation theory and in the study of moduli spaces of vector bundles on algebraic curves. However, a reasonable algebro-geometric structure on the set $\mathrm{GL}_{n}\left(\mathbb{Q}_{p}\right) / \mathrm{GL}_{n}\left(\mathbb{Z}_{p}\right)$ is not available for many years, although some attempts have been made ([Ha, $\mathrm{Kr}$, $\mathrm{CKV}$ ]). The first goal of this paper is to give a solution of this problem to some extent, and we will call this algebro-geometric object the mixed characteristic affine Grassmannian, or just the affine Grassmannian, if it is clear we are working in mixed characteristic.

0.1.2. To explain the ideas, let us first recall the equal characteristic story (see, e.g. BL] for details). First one can make sense of the notion of a family of lattices in $k((t))^{n}$ : let $R$ be a $k$-algebra, a lattice in $R((t))^{n}$ is a finite generated projective $R[[t]]$-submodule $\Lambda$ of $R((t))^{n}$ such that $\Lambda \otimes_{R[[t]]} R((t))=R((t))^{n}$. Then the affine Grassmannian Gr 1 is the moduli space that assigns every $k$-algebra $R$, the set of lattices in $R((t))^{n}$. In particular, the set of $k$-points of the functor is just $\mathrm{GL}_{n}(k((t))) / \mathrm{GL}_{n}(k[[t]])$.

It is easy to see that given a lattice $\Lambda$, there always exists some $N$ large enough such that

$$
t^{N} R[[t]]^{n} \subset \Lambda \subset t^{-N} R[[t]]^{n} .
$$

So $\mathrm{Gr}^{b}$ is the union of subfunctors $\mathrm{Gr}^{b,(N)}$ consisting of those lattices satisfying (0.1.1). The key fact, that allows one to prove the representability of $\mathrm{Gr}^{\mathrm{b}}$, is that via the map

$$
\Lambda \mapsto t^{-N} R[[t]]^{n} / \Lambda
$$

$\mathrm{Gr}^{b,(N)}(R)$ is identified with the set of quotients $R[[t]]-$ modules of $t^{-N} R[[t]]^{n} / t^{N} R[[t]]^{n}$ that are projective as $R$-modules. Then it is clear that $\mathrm{Gr}^{\mathrm{b},(N)}$ is represented by a closed subscheme of the usual Grassmannian.

0.1.3. There is an obvious guess of the moduli problem that should be represented by the mixed characteristic affine Grassmannian: we associate to every $k$-algebra $R$ the set

$\operatorname{Gr}(R)=\left\{\Lambda\right.$ finite projective $W(R)$-submodules of $W(R)[1 / p]^{n}$ satisfying $\left.\Lambda[1 / p]=W(R)[1 / p]^{n}\right\}$,

where $W(R)$ is the ring of Witt vectors for $R$. Unfortunately, this definition is unreasonable, as the ring of the Witt vectors for a non-perfect $\operatorname{ring} R$ is not well-behaved. E.g. $p$ could be a zero divisor of $W(R)$ if $R$ is non-reduced so $\Lambda_{0}=W(R)^{n}$ may not be in the above set 2 . On the other hand, note that

(1) if $R$ is a perfect $k$-algebra, then $W(R)$ is well-behaved.

\footnotetext{
${ }^{1}$ In this paper, the objects defined in equal characteristic setting are usually written with $b$ as its supscript. But this notation does not suggest any deep relation with P. Scholze's theory of perfectoid spaces.

${ }^{2}$ Alternatively, one could try to define $\operatorname{Gr}(R)$ as the set of pairs $(\Lambda, \beta)$ where $\Lambda$ is a finite projective $W(R)$-module and $\beta: \Lambda \otimes W(R)[1 / p] \simeq W(R)[1 / p]^{n}$ is an isomorphism. But we still do not know whether this is reasonable.
} 
(2) The $R$-points of a scheme $X$ for perfect rings $R$ determine $X$ up to perfection 3 .

(3) The (étale) topology of a scheme (e.g. the $\ell$-adic cohomology) does not change passing to perfection.

Therefore, we restrict the naive moduli problem (0.1.2) to the category of perfect $k$ algebras. This defines a presheaf on this category, and the best question one can ask is: whether this functor is represented by a(n inductive limit of) perfect $k$-scheme(s). Our first main theorem gives a positive answer of the question.

Theorem 0.1. The above functor can be written as $\mathrm{Gr}=\lim _{i} X_{i}$, with each $X_{i}$ being the perfection of some proper algebraic space defined over 15 , and $X_{i} \rightarrow X_{i+1}$ being a closed embedding.

Perfect $k$-schemes are almost never of finite type over $k$. But as claimed in Theorem 0.1 in fact each $X_{i}$ appearing above is the perfection of some proper algebraic space over $k$. Note that we do not know how to canonically construct these algebraic spaces without passing to the perfection. But this does not bother us. We can still study their topological properties. In particular, we can define the $\ell$-adic derived category on $X_{i}$, the notion of perverse sheaves, and etc.

As soon as the representability of $\mathrm{Gr}$ is proved, the representabilities of mixed characteristic affine Grassmannians and affine flag varieties for general reductive groups follow by an argument same as equal characteristic situation. See $\S 1.5$

0.1.4. Now we explain some more details how to prove the representability of Gr. As in equal characteristic situation, it is enough to prove the subfunctor $\mathrm{Gr}^{(N)}$ of $\mathrm{Gr}$ defined by a condition similar to (0.1.1) is representable. A little further work shows that it is enough to prove the representability of

$$
\overline{\operatorname{Gr}}_{N}=\left\{(\mathcal{E}, \beta) \in \operatorname{Gr} \mid \beta: \mathcal{E} \rightarrow \mathcal{E}_{0} \text { induces } \wedge^{n} \beta: \wedge^{n} \mathcal{E} \simeq p^{N} \wedge^{n} \mathcal{E}_{0}\right\},
$$

where $\mathcal{E}_{0}=W(R)^{n}$. Now, the essential difficulty is that the quotient $\mathcal{E}_{0} / \mathcal{E}$ cannot be regarded as an $R$-module and it is not clear how to embed this functor into the Grassmannian (or projective spaces). An even more basic (and very interesting) question is, whether there exists a non-trivial line bundle on $\overline{\mathrm{Gr}}_{N}$. Note that in equal characteristic, such a line bundle exists and is ample, known as the determinant line bundle $\mathcal{L}_{\text {det }}^{b}$ on $\overline{\mathrm{Gr}}_{N}^{b}$. Its fiber over $(\mathcal{E}, \beta)$ is the top exterior wedge of $\mathcal{E}_{0} / \mathcal{E}$ as an $R$-module. This construction certainly fails in mixed characteristic. (See Appendix B.1 for some discussions.)

Therefore, we proceed in another way. Our observation is that by adding certain level structure, $\overline{\mathrm{Gr}}_{N}$ is indeed representable by some affine scheme defined by matrix equations. More precisely, for each $h$, let $W_{h}(R)$ denote the ring of truncated Witt vectors of length $h$, and let

$$
\overline{\operatorname{Gr}}_{N, h}=\left\{\left.(\mathcal{E}, \beta, \bar{\epsilon})\left|(\mathcal{E}, \beta) \in \overline{\mathrm{Gr}}_{N}, \bar{\epsilon}: \mathcal{E}_{0}\right|_{W_{h}(R)} \simeq \mathcal{E}\right|_{W_{h}(R)}\right\} .
$$

This is an $L^{h} \mathrm{GL}_{n}$-torsor over $\overline{\mathrm{Gr}}_{N}$. Here $L^{h} \mathrm{GL}_{n}$ is the perfection of the Greenberg realization of $\mathrm{GL}_{n}$ over $\mathcal{O} / p^{h}$ (which is an affine group scheme over $k$ ), and it acts on $\overline{\mathrm{Gr}}_{N, h}$ by changing the trivialization $\bar{\epsilon}$. We will show that when $h>N, \overline{\mathrm{Gr}}_{N, h}$ can be (non-canonically) identified with the following scheme of pairs of matrices

$$
\left\{(A, \gamma) \mid \gamma \in L^{h} \mathrm{GL}_{n}, A \in L^{h} M_{n}, \operatorname{det} A \in p^{N}\left(\mathcal{O} / p^{h} \mathcal{O}\right)^{\times}, A \gamma=A\right\},
$$

where $M_{n}$ is the scheme of all $n \times n$ matrices. In fact, $A=\left(\left.\beta\right|_{W_{h}(R)}\right) \bar{\epsilon}$.

\footnotetext{
${ }^{3}$ The category of perfect $k$-schemes is a full subcategory of the category of presheaves on the category of perfect $k$-algebras, see Lemma A.10

${ }^{4} \mathrm{In}$ fact, in $[\mathrm{Kr}]$ it is shown that this is an fpqc sheaf.

${ }^{5}$ One can expect that these $X_{i}$ s are the perfections of projective varieties over $k$, see $\mathrm{B}$ for some discussions. But knowing $X_{i}$ s are algebraic spaces is sufficient for all the applications we have in mind.
} 
Therefore, $\overline{\mathrm{Gr}}_{N}$ can be expressed as a quotient of an affine scheme by a free action of an affine group scheme. In general, one can expect such a quotient should exist at least as an algebraic space over $k$. This is indeed the case here, but cannot follow directly from the general theory, because neither $\overline{\mathrm{Gr}}_{N, h}$ nor $L^{h} \mathrm{GL}_{n}$ are of finite type (in fact the usual construction of the étale slices, which uses the sheaf of differentials, does not work for perfect schemes). However, we are managing to prove the following result, which is enough to deduce the representability of $\overline{\mathrm{Gr}}_{N}$ as an algebraic space. Interestingly, the proof uses the construction of coarse space for certain algebraic stacks.

Proposition 0.2. (See Proposition A.28) Let $G$ be the perfection of an affine group scheme of finite type over $k$ and $X$ be the perfection of a scheme of finite type over $k$. Let $\sigma$ : $G \times X \rightarrow X$ be an action of $G$ on $X$ and assume that the action map $a=\left(\sigma, \mathrm{pr}_{2}\right): G \times X \rightarrow$ $X \times X,(g, x) \mapsto(g x, x)$ is a closed embedding. Then the quotient $[X / G]$ (as a groupoid) is represented by the perfection of an algebraic space separated and of finite type over $k$.

0.2. The geometric Satake. The representability of mixed characteristic affine Grassmannians has a lot of applications. The most fundamental application is to establish the geometric Satake correspondence in mixed characterisitc. Its equal characteristic counterpart is the result of works of Lusztig, Ginzburg, Beilinson-Drinfeld, Mirković-Vilonen ([Lu1, Gi, BD, MV] ). In a forthcoming joint work with L. Xiao [XZ], we will see applications of the mixed characteristic geometric Satake to the arithmetic geometry of Shimura varieties 6 .

Let $G$ be a reductive group over $\mathcal{O}$, the ring of integers of a $p$-adic field $F$ and let $\operatorname{Gr}_{G}$ denote its affine Grassmannian. As explain above, it makes sense to talk about the category of $L^{+} G$-equivariant perverse sheaves (with coefficients in $\overline{\mathbb{Q}}_{\ell}$ ) on $\mathrm{Gr}_{G}$, denoted by $\mathrm{P}_{L+G}\left(\mathrm{Gr}_{G}\right)$. As in the equal characteristic situation, this is a semisimple category and Lusztig's convolution of sheaves give a natural monoidal structure on $\mathrm{P}_{L^{+} G}\left(\mathrm{Gr}_{G}\right)$. In addition, one can still endow the hypercohomology functor $\mathrm{H}^{*}\left(\mathrm{Gr}_{G},-\right): \mathrm{P}_{L^{+} G}\left(\mathrm{Gr}_{G}\right) \rightarrow$ Vect $_{\overline{\mathbb{Q}}_{\ell}}$ with a canonical monoidal structure (although the methods used in [Gi, BD, MV] do not work directly in our setting). Our second main theorem, the geometric Satake correspondence, claims that

Theorem 0.3. The monoidal functor $\mathrm{H}^{*}$ factors as the composition of an equivalence of monoidal categories from $\mathrm{P}_{L^{+} G}\left(\mathrm{Gr}_{G}\right)$ to the category $\operatorname{Rep}_{\overline{\mathbb{Q}}_{\ell}}(\hat{G})$ of finite dimensional representations of the Langlands dual group $\hat{G}$ over $\overline{\mathbb{Q}}_{\ell}$ and the forgetful functor $\operatorname{Rep}_{\overline{\mathbb{Q}}_{\ell}}(\hat{G}) \rightarrow$ $\operatorname{Vect}_{\overline{\mathbb{Q}}_{\ell}}$.

The theorem in particular implies that there exist the commutativity constraints of $\mathrm{P}_{L^{+} G}\left(\mathrm{Gr}_{G}\right)$ such that $\mathrm{H}^{*}$ is a tensor functor. In the equal characteristic situation, such constraints come from the interpretation of convolution products as fusion products (cf. [MV, §5] or [BD, §5.3.17]). It is unlikely that the fusion product could exist in mixed characteristic so it is probably surprising that we can still establish these constraints in the current setting.

In fact, a construction of the commutativity constraints using a categorical version of the classical Gelfand's trick already appeared in [Gi]. It was then claimed in [BD, $\S 5.3 .8$, §5.3.9] (but without proof) that (a modification of) Ginzburg's constraints coincide with the commutativity constraints from fusion products. Therefore, we do have candidates of the commutativity constraints even in mixed characteristic. The problem is that it is not clear how to verify the properties they suppose to satisfy, without using the fusion interpretation.

Our new observation is that the validity of these properties is equivalent to a combinatoric formula in the theory of affine Hecke algebras. Namely, in [LV, Lu2, Lusztig and Vogan introduced, for a Coxeter system $(W, S)$ with an involution, certain polynomials $P_{y, w}^{\sigma}(q)$,

\footnotetext{
${ }^{6}$ We refer to Laf for some amazing applications of equal characteristic geometric Satake to Langlands correspondence over function fields.
} 
similar to the usual Kazhdan-Lusztig polynomials $P_{y, w}(q)$ ([KL1]). Then it was conjectured in Lu2 that if $(W, S)$ is an affine Weyl group and $y, w$ are certain elements in $W$,

$$
P_{y, w}^{\sigma}(q)=P_{y, w}(-q) \text {. }
$$

See loc. cit. or $\$ 2.4 .5$ for more details. This conjecture is purely combinatoric, but its proof by Lusztig and Yun [LY] is geometric, and in fact uses the equal characteristic geometric Satake! We then go the opposite direction by showing that this formula implies the above mentioned commutativity constraints are the correct ones.

So our proof uses the geometric Satake in equal charateristi. It is an interesting question to see whether it is possible to prove this combinatoric formula directly 7 , which will yield a pure local proof of the geometric Satake, in both equal and mixed characteristic.

In the course of the proof, we will also establish the Mirković-Vilonen theory in mixed characteristic. This theory is very useful to study affine Deligne-Lusztig varieties (cf. [XZ]).

0.3. Dimension of affine Deligne-Lusztig varieties. One motivation to construct mixed characteristic affine Grassmannians is their relation to Rapoport-Zink spaces. Let $G$ be a connected reductive group over $\mathbb{Q}_{p}$, and assume (for simplicity) that there is an extension of $G$ to a reductive group scheme over $\mathbb{Z}_{p}$, still denoted by $G$. Let $k=\overline{\mathbb{F}}_{p}$ and let $L=$ $W(k)[1 / p]$ denote the completion of the maximal unramified extension of $\mathbb{Q}_{p}$. Let $\sigma$ denote the Frobenius automorphism of $L$. Let $b$ be a $\sigma$-conjugacy class of $G(L)$ and $\mu$ be a geometric conjugacy class of one parameter subgroups of $G$ (a.k.a a dominant coweight of $G$ w.r.t. some chosen Borel). When the triple $(G, b, \mu)$ (which we call a Rapoport-Zink datum) comes from a PEL-datum, Rapoport-Zink (cf. [RZ]) defined a formal scheme $\breve{\mathcal{M}}(G, b, \mu)$, locally of finite type over $W=W(k)$, as certain moduli problem of $p$-divisible groups. Recently, Kim (cf. Kim) generalized the definition of $\breve{\mathcal{M}}(G, b, \mu)$ for those Rapoport-Zink data of Hodge type and proved the representability of $\breve{\mathcal{M}}(G, b, \mu)$ in the case $p>2$. In any case, a serious restriction of $\mu$ is that it must be minuscule. Then the Dieudonné theory identifies the set of $k$-points of $\breve{\mathcal{M}}(G, b, \mu)$ with

$$
X_{\mu}(b)=\left\{g \in G(L) / G(W) \mid g^{-1} b \sigma(g) \in G(W) p^{\mu} G(W)\right\} .
$$

This identification endows $X_{\mu}(b)$ with some algebro-geometric structure, sometimes also called the (mixed characteristic) affine Deligne-Lusztig variety. Note that it is possible to define $X_{\mu}(b)$ as a set for any triple $(G, b, \mu)$ but only for minuscule $\mu, X_{\mu}(b)$ may relate to the moduli of $p$-divisible groups and therefore may acquire a scheme structure.

It is hoped to endow $X_{\mu}(b)$ with an algebro-geometric structure without using the $p$ divisible groups and the Dieudonné theory. Now, the existence of the mixed characteristic affine Grassmannian $\operatorname{Gr}_{G}$ (for $G$ over a $p$-adic field $F$ ) allows us to realize $X_{\mu}(b)$ as a (locally) closed subset of $\mathrm{Gr}_{G}$ and therefore to give $X_{\mu}(b)$ a structure as a(n ind) perfect algebraic space. Note that in this new definition, there is no restriction on the cocharacter $\mu$. But when $(G, b, \mu)$ arise as a(n unramified) Rapoport-Zink datum of Hodge type as above, we have the following proposition, as a simple application of the equivalence of categories between $p$-divisible groups and the $F$-crystals over a perfect ring in characteristic $p>0$ (a theorem of Gabber, see also [La, §6]).

Proposition 0.4. Let $\overline{\mathcal{M}}_{\mu}^{p^{-\infty}}(b)$ denote the perfection of the special fiber of $\breve{\mathcal{M}}(G, b, \mu)$. Then there is a canonical isomorphism of spaces $\overline{\mathcal{M}}_{\mu}^{p^{-\infty}}(b) \simeq X_{\mu}(b)$.

Even if the primary interests are the study of the Rapoport-Zink spaces, having another definition of $X_{\mu}(b)$ gives us extra flexibility. For example, the new definition is group theoretically, so allows us to study $\breve{\mathcal{M}}(G, b, \mu)$ by using root subgroups or Levi sbugroups $G$, or passing to central isogenies of $G$.

\footnotetext{
${ }^{7}$ This seems possible if the affine Weyl group is of type $A$.
} 
In the forthcoming work XZ, these extra flexibilities allow us to understand the irreducible components of certain RZ spaces. Here, we illustrate the usefulness of them by one simple example8: We prove the dimension formula of $X_{\mu}(b)$ as conjectured by Rapoport.

Theorem 0.5. The ind perfect algebraic space $X_{\mu}(b)$ is finite dimensional, and

$$
\operatorname{dim} X_{\mu}(b)=\left\langle\rho, \mu-\nu_{b}\right\rangle-\frac{1}{2} \operatorname{def}_{G}(b) .
$$

We refer to $\S 3.1$ for unexplained notations. Thanks to Proposition 0.4 , when $(G, b, \mu)$ is of Hodge type, we get the dimension formula of the corresponding Rapoport-Zink space 9 .

Not surprisingly, after the machinery is set up, we can imitate the methods used in the equal characteristic situation (with one innovation): the arguments of GHKR apply here and reduce to prove Theorem 0.5 for those $b$ that are the so-called superbasic $\sigma$-conjugacy classes. It was shown in GHKR, CKV that if $G$ is of adjoint type, superbasic $\sigma$-conjucay classes exist only when $G=\mathrm{PGL}_{n}$ or $G=\operatorname{Res}_{E / F} \mathrm{PGL}_{n}$, where $E / F$ is an unramified extension. The case when $G=\mathrm{PGL}_{n}$ was treated in V1 (in the equal characteristic situation but the same arguments apply here). The case $G=\operatorname{Res}_{E / F} \mathrm{PGL}_{n}$ was treated in Ham2 in the equal characteristic situation and then was adapted in [Ham1 to deal with the corresponding Rapoport-Zink spaces. Our innovation here is a reduction of the case $G=\operatorname{Res}_{E / F} \mathrm{PGL}_{n}$ to the case $G=\mathrm{PGL}_{n}$ case so one can apply [V1] directly (see Proposition 3.4). This in particular gives a much shorter proof of the main result of [Ham2] (assuming [V1]). We mention that this reduction step uses the representability of $X_{\mu}(b)$ for non-minuscule $\mu$ (even we just interested in proving Theorem 0.5 for Rapoport-Zink spaces), and also the semismallness of the convolution products for affine Grassmannians.

0.4. Organization. We quickly discuss the organization of this paper. In \$1 we prove the representability of affine Grassmannians and affine flag varieties and discuss their first properties. We establish the geometric Satake correspondence in \$2. In \$3, we prove the dimension formula for Rapoport-Zink spaces, conjectured by Rapoport, as an application of our theory. The paper contains two appendices. Appendix A discusses perfect schemes and perfect algebraic spaces in some generality, which is the setting we will work with in the paper. Appendix B] discusses some further questions on affine Grassmannians, including conjectures related to the representability of affine Grassmannians as schemes and deperfections of the "Schubert variety" inside them. We also give an example of the construction in 1 .

0.5. Notations. We fix $k$ a perfect field of characteristic $p>0$. For a $k$-algebra $R$, let

$$
W(R)=\left\{\left(a_{0}, a_{1}, \ldots\right) \mid a_{i} \in R\right\}
$$

denote its ring of Witt vectors, and let $R \rightarrow W(R), x \mapsto[x]=(x, 0,0, \ldots)$ be the Teichmüller lifting. We denote by $W_{h}(R)$ the ring of truncated Witt vectors of length $h$. If $R$ is perfect, $W_{h}(R)=W(R) / p^{h} W(R)$.

Let us write $\mathcal{O}_{0}=W(k), F_{0}=W(k)[1 / p]$. Except $3.2, F$ denotes a finite totally ramified extension of $F_{0}$ and $\mathcal{O}$ its ring of integers. Let $\varpi$ denote a uniformizer of $\mathcal{O}$. For a $k$-algebra $R$, let $W_{\mathcal{O}}(R)=W(R) \otimes_{W(k)} \mathcal{O}$ and $W_{\mathcal{O}, n}(R)=W_{n}(R) \otimes_{W(k)} \mathcal{O}$. In particular, we write $W_{\mathcal{O}}(\bar{k})=\mathcal{O}_{L}$ and $L=\mathcal{O}_{L}[1 / p]$ the completion of the maximal unramified extension of $F$.

Unless specified, $G$ will denote a reductive group scheme over $\mathcal{O}$. If $G$ is split, we will fix a Borel subgroup $B$, its unipotent radical $U$, and a maximal torus $T \subset B$. Sometimes, we denote by $\bar{G}, \bar{B}, \bar{U}, \bar{T}$ their reduction modulo $\varpi$. Let $\mathbb{X} \bullet$ denote the coweight lattice

\footnotetext{
${ }^{8}$ An earlier example is given by [CKV] to study the connected components of $X_{\mu}(b)$, although they must define the notion of connected components of $X_{\mu}(b)$ in an ad hoc way, due to lacking of the representability of $X_{\mu}(b)$ at that time.

${ }^{9}$ When the first draft version of the paper was completed, Hamacher released his preprint Ham1] where Rapoport's conjecture for PEL type Rapoport-Zink spaces was solved.
} 
of $T$, and $\mathbb{X}_{\bullet}^{+}$denote the semi-group of dominant coweights. For $\lambda \in \mathbb{X}_{\bullet}$, the image of $\varpi \in F^{\times}=\mathbb{G}_{m}(F)$ under $\lambda: \mathbb{G}_{m} \rightarrow T \rightarrow G$ is denoted by $\varpi^{\lambda}$.

Let " $\preceq$ " be the partial order on $\mathbb{X}_{\bullet}: \lambda \preceq \mu$ if $\mu-\lambda$ is a linear combination of positive roots (w.r.t. $B$ ) with coefficients $\in \mathbb{Z}_{\geq 0}$. The restriction of this partial order to $\mathbb{X}_{\bullet}^{+}$is sometimes also denoted by " $\leq$ ". The dual group of $G$ (over a field of characteristic zero) is denoted by $\hat{G}$. For $\mu \in \mathbb{X}_{\bullet}^{+}$, let $V_{\mu}$ denote the irreducible representation of $\hat{G}$ of highest weight $\mu$. For $\lambda \in \mathbb{X}_{\bullet}$, let $V_{\mu}(\lambda)$ denote the $\lambda$-weight subspace of $V_{\mu}$. We denote by $2 \rho$ the sum of positive roots.

0.6. Acknowledgement. An ongoing project with L. Xiao is the main motivation to develop the theories in the current paper. The author thanks him cordially for the collaboration. The author also thanks B. Conrad, V. Drinfeld, B. Elias, A. de Jong, X. He, J. Kamnitzer, G. Pappas and Z. Yun for useful discusssions and comments. He in particular would like to thank J. Kamnitzer for pointing out a serious mistake in an early draft of the paper. The author is partially supported by NSF grant DMS-1001280/1313894 and DMS-1303296 and the AMS Centennial Fellowship.

\section{Affine Grassmannians}

In this section, we construct affine Grassmannians and affine flag varieties in mixed characteristic. We refer to Appendix $\AA$ for the necessary background of perfect algebraic spaces, which is the setting we will work with in the sequel.

1.1. $p$-adic loop groups. We refer 80.5 for the notations. Let $\mathcal{X}$ be an $\mathcal{O}$-scheme, of finite type. We define

$$
L_{p}^{+} \mathcal{X}(R)=\mathcal{X}\left(W_{\mathcal{O}}(R)\right), \quad L_{p}^{h} \mathcal{X}(R)=\mathcal{X}\left(W_{\mathcal{O}, h}(R)\right)
$$

It is well-known from Greenberg (Grb) that they are represented by schemes over $k$. In addition, each $L_{p}^{h} \mathcal{X}$ is of finite type, and $L_{p}^{+} \mathcal{X}=\lim _{\longleftarrow} L_{p}^{h} \mathcal{X}$. If $\mathcal{X} \subset \mathcal{Y}$ is open, then $L_{p}^{+} \mathcal{X} \subset$ $L_{p}^{+} \mathcal{Y}$ is open. We denote their perfections by

$$
L^{+} \mathcal{X}=\left(L_{p}^{+} \mathcal{X}\right)^{p^{-\infty}}, \quad L^{h} \mathcal{X}=\left(L_{p}^{h} \mathcal{X}\right)^{p^{-\infty}}
$$

The justification of the choice of the notations is that perfect objects behave better and are more similar to their equal characteristic analogues. If $f: \mathcal{X} \rightarrow \mathcal{Y}$ is an $\mathcal{O}$-map, we denote by $L_{p}^{+} f: L_{p}^{+} \mathcal{X} \rightarrow L_{p}^{+} \mathcal{Y}$ and $L^{+} f: L^{+} \mathcal{X} \rightarrow L^{+} \mathcal{Y}$ the induced maps.

Let $X$ be an affine scheme over $F$. We define a perfect space by assigning a perfect $k$-algebra $R$,

$$
L X(R)=X\left(W_{\mathcal{O}}(R)[1 / p]\right) .
$$

If $f: X \rightarrow Y$ is an $F$-map, we have $L f: L X \rightarrow L Y$. Unlike [ $\mathrm{Kr}$, we do not define the object $L_{p} X$. According to Lemma A.10, the following statement makes sense.

Proposition 1.1. Assume that $X$ is affine of finite type, then $L X$ is represented by an ind perfect schemes.

Proof. As soon as we go to perfection, the proof is similar to the representability of the usual loop groups in equal characteristic setting.

First, it is enough to consider $F=F_{0}=W(k)[1 / p]$. If $X=\mathbb{A}^{1}$, then $L X=\lim _{\longrightarrow}\left(\mathbb{A}^{\infty}\right)^{p^{-\infty}}$. This follows from the fact that every element in $W(R)[1 / p]$ can be uniquely written as

$$
x=\sum_{i \geq-N} p^{i}\left[x_{i}\right] .
$$

Second, $X=X_{1} \times X_{2}$, then $L\left(X_{1} \times X_{2}\right)=L X_{1} \times L X_{2}$ so $L \mathbb{A}^{n}$ is representable. Finally, if $Z \subset \mathbb{A}^{n}$ is a closed embedding, then $L Z \rightarrow L \mathbb{A}^{n}$ is a closed embedding. Indeed we can write

$$
[x]+[y]=\sum p^{j}\left[\Sigma_{j}(x, y)^{1 / p^{j}}\right]
$$


where $\Sigma_{j}(X, Y) \mathrm{s}$ are certain polynomials with two indeterminants $X, Y$, of homogeneous degree $p^{j}$. Now assume that

$$
\mathcal{O}_{Z}=F\left[t_{1}, \ldots, t_{n}\right] /\left(f_{1}, \ldots, f_{m}\right) .
$$

It is easy to see that if $f\left(t_{1}, \ldots, t_{n}\right)$ is a polynomial with coefficients in $F$, then

$$
f\left(\sum p^{i}\left[x_{1 i}\right], \ldots, \sum p^{i}\left[x_{n i}\right]\right)=\sum p^{j}\left[f^{(j)}\left(x_{m l}\right)^{1 / p^{j}}\right] .
$$

where $f^{(j)}$ s are some polynomials in terms of the variables $X_{m l}, m=1, \ldots, n, l \in \mathbb{Z}$. Then $L_{p} Z$ is defined in $L_{p} \mathbb{A}^{n}$ by the equations $f_{r}^{(j)},\left(f_{r}^{(j)}\right)^{1 / p},\left(f_{r}^{(j)}\right)^{1 / p^{2}}, \ldots$

It is also clear from the above argument that

Lemma 1.2. (i) Assume that $\mathcal{X}$ is affine of finite type, then $L^{+} \mathcal{X} \rightarrow L X$ is a closed embedding.

(ii) If $X \rightarrow Y$ is a closed embedding, then $L X \rightarrow L Y$ is a closed embedding.

Now, let $\mathcal{X}=G$ be a smooth affine group scheme over $\mathcal{O}$. We write $G^{(0)}=G$ and define the $h$ th congruence group scheme of $G$ over $\mathcal{O}$, denoted by $G^{(h)}$, as the dilatation of $G^{(h-1)}$ along the unit. There is a natural map $G^{(h)} \rightarrow G$ and note that

$$
L^{+} G^{(h)}=\operatorname{ker}\left(L^{+} G \rightarrow L^{h} G\right) .
$$

However, $L_{p}^{+} G^{(h)} \neq \operatorname{ker}\left(L_{p}^{+} G \rightarrow L_{p}^{h} G\right)$.

\subsection{The affine Grassmannian for $\mathrm{GL}_{n}$.}

1.2.1. Let $G$ be a reductive group over $\mathcal{O}$. We define the affine Grassannian of $G$ as the perfect space

$$
\operatorname{Gr}_{G}:=\left[L G / L^{+} G\right] .
$$

See $\S$ A.1.4 for the notation. Explicitly, for a perfect $k$-algebra $R, \operatorname{Gr}_{G}(R)$ is the set of pairs $(P, \phi)$, where $P$ is an $L^{+} G$-torsor on $\operatorname{Spec} R$ and $\phi: P \rightarrow L G$ is an $L^{+} G$-equivariant morphism. As in equal characteristic situation, there is another interpretation of $\operatorname{Gr}_{G}$ (e.g. see $[\overline{\mathrm{Kr}}]$ ). Denote by $\mathcal{E}_{0}$ the trivial $G$-torsor on $\mathcal{O}$.

Lemma 1.3. We have

$$
\operatorname{Gr}_{G}=\left\{\begin{array}{l|l}
(\mathcal{E}, \beta) & \begin{array}{l}
\mathcal{E} \text { is a } G \text {-torsor on } W_{\mathcal{O}}(R), \text { and } \\
\beta: \mathcal{E}[1 / p] \simeq \mathcal{E}_{0}[1 / p] \text { is a trivialization }
\end{array}
\end{array}\right\} .
$$

Remark 1.4. Recall that for a perfect ring $R$, a finite projective $W(R)$-module is the same as a locally free crystal on $R$. Such $\beta$ sometimes is called a quasi-isogeny. Then the above lemma can also be interpreted by saying that if $F=F_{0}, \mathrm{Gr}_{G}$ classifies a crystal with $G$-structure on $R$ together with a quasi-isogeny to the trivial $G$-crystal.

According to Lemma A.10, one can ask whether $\mathrm{Gr}_{G}$ is representable by perfect (ind)schemes. Our main theorem of this section is

Theorem 1.5. The affine Grassmannian $\mathrm{Gr}_{G}$ is represented by an ind perfectly proper perfect algebraic space.

Again, as in the equal characteristic situation, we can reduce the proof of this theorem to the case $G=\mathrm{GL}_{n}$ and $F=F_{0}$ (see Proposition $\left[1.20\right.$ ). So we will concentrate on $\mathrm{GL}_{n}$ case first. We will denote $\mathrm{Gr}_{\mathrm{GL}_{n}}$ by $\mathrm{Gr}$ in this subsection for simplicity. 
1.2.2. Let us write the moduli problem Gr more explicitly: Let $\mathcal{E}_{0}=W(R)^{n}$ denote the free $W(R)$-module of rank $n$. For a perfect $k$-algebra $R$,

$$
\operatorname{Gr}(R)=\left\{\begin{array}{l|l}
(\mathcal{E}, \beta) & \begin{array}{l}
\mathcal{E} \text { is a rank } n \text { projective } W(R) \text {-module, } \\
\beta: \mathcal{E}[1 / p] \rightarrow \mathcal{E}_{0}[1 / p] \text { is a trivialization }
\end{array}
\end{array}\right\} .
$$

Via $\beta$, we can regard $\mathcal{E}$ as a finitely generated projective $W(R)$-submodule $\Lambda$ of $W(R)[1 / p]^{n}=$ $\mathcal{E}_{0}[1 / p]$ such that $\Lambda \otimes_{W(R)} W(R)[1 / p]=W(R)[1 / p]^{n}$. Such $\Lambda$ sometimes is called a lattice, whose basic properties now we recall.

Let $k$ be a perfect field, and let $E_{1}$ and $E_{2}$ be two finite free $W(k)$-modules of rank $n$. Let $\beta: E_{1}[1 / p] \simeq E_{2}[1 / p]$ be an isomorphism, usually called a quasi-isogeny between $E_{1}$ and $E_{2}$. It then makes sense to talk about the relative position of $\beta$, $\operatorname{denoted} \operatorname{Inv}(\beta)$, which is an element in

$$
\mathbb{X}_{\bullet}\left(D_{n}\right)^{+}=\left\{\lambda=\left(m_{1}, \ldots, m_{n}\right) \in \mathbb{Z}^{n} \mid m_{1} \geq m_{2} \geq \cdots \geq m_{n}\right\},
$$

the semigroup of dominant coweights of $\mathrm{GL}_{n} 10$. Namely, there always exists basis $\left(e_{1}, \ldots, e_{n}\right)$ of $E_{1}$ and $\left(f_{1}, \ldots, f_{n}\right)$ of $E_{2}$ such that $\beta$ is given by $\beta\left(e_{i}\right)=p^{m_{i}} f_{i}$ and $m_{1} \geq m_{2} \geq \cdots \geq m_{n}$. In addition, this sequence $\left(m_{1}, \ldots, m_{n}\right)$ is independent of the choice of the basis.

We denote by $\omega_{i}=(1, \ldots, 1,0, \ldots, 0)$ with the first $i$ entries 1 and the last $n-i$ entries 0 . Let $\omega_{i}^{*}=\omega_{n}-\omega_{n-i}$. Note that $\operatorname{inv}(\beta)=\omega_{i}$ if and only if $\beta$ extends to $E_{1} \rightarrow E_{2}$ is a map such that $p E_{2} \subset E_{1}$ and $E_{2} / E_{1}$ is a $k$-vector space of dimension $i$. $\operatorname{Similarly,~} \operatorname{inv}(\beta)=\omega_{i}^{*}$ if and only if $\beta$ extends to $p E_{1} \subset E_{2} \subset E_{1}$ such that $E_{1} / E_{2}$ is of dimension $i$.

Recall that $\mathbb{X}_{\bullet}\left(D_{n}\right)^{+}$has a partial order $\leq$: Let

$$
\alpha_{1}=(1,-1,0, \ldots, 0), \alpha_{2}=(0,1,-1,0, \ldots, 0), \ldots, \alpha_{n-1}=(0, \ldots, 0,1,-1) .
$$

Then $\lambda_{1} \leq \lambda_{2}$ if $\lambda_{2}-\lambda_{1}$ is non-negative a linear combination of $\alpha_{i}$ s. Note that minimal elements under this partial order are of the form $\omega_{i}+m \omega_{n}$. In particular, $\underline{0}:=0 \omega_{n}$ is a minimal element.

Let $R$ be a perfect $k$-algebra. Let $\mathcal{E}_{1}$ and $\mathcal{E}_{2}$ be two finite projective $W(R)$-modules, and $\beta: \mathcal{E}_{1}[1 / p] \rightarrow \mathcal{E}_{2}[1 / p]$ be an isomorphism. If $x \in \operatorname{Spec} R$, we denote by $\beta_{x}: \mathcal{E}_{1} \otimes_{W(R)}$ $W(k(x))[1 / p] \rightarrow \mathcal{E}_{2} \otimes_{W(R)} W(k(x))[1 / p]$. We denote by

$$
(\operatorname{Spec} R)_{\lambda}=\left\{x \in \operatorname{Spec} R \mid \operatorname{Inv}\left(\beta_{x}\right)=\lambda\right\} \subset(\operatorname{Spec} R)_{\leq \lambda}=\left\{x \in \operatorname{Spec} R \mid \operatorname{Inv}\left(\beta_{x}\right) \leq \lambda\right\} .
$$

Lemma 1.6. Let $\beta: \mathcal{E}_{1}[1 / p] \rightarrow \mathcal{E}_{2}[1 / p]$ be a quasi-isogeny over $R$. Assume that $\operatorname{Spec} R=$ $(\operatorname{Spec} R)_{\omega_{i}}$. Then we have $p \mathcal{E}_{2} \subset \mathcal{E}_{1} \subset \mathcal{E}_{2}$ and $\mathcal{E}_{2} / \mathcal{E}_{1}$ is a finte projective $W(R) / p=R$ module of rank $i$. A similar statement holds for $\omega_{i}^{*}$.

Proof. Let $\beta: \mathcal{E}_{1}[1 / p] \rightarrow \mathcal{E}_{2} 1[/ p]$ be a quasi-isogeny of finite projective $W(R)$-modules. We claim that if at every $x \in R, \beta_{x}$ is a genuine map, then $\beta$ is a genuine map. Indeed, there is an open cover $\operatorname{Spec} W(R)=\cup \operatorname{Spec} W(R)_{f_{i}}$ such that both $\mathcal{E}_{1}$ and $\mathcal{E}_{2}$ are free so that we can represent $\beta$ by an element in $A_{i} \in M_{n \times n}\left(W(R)_{f_{i}}[1 / p]\right)$. We need to show that $A_{i} \in M_{n \times n}\left(W(R)_{f_{i}}\right)$. Let $\bar{f}_{i}=f_{i} \bmod p$. Then we have $j: W(R)_{f_{i}} \rightarrow W\left(R_{\bar{f}_{i}}\right)$ and it is enough to show that $j\left(A_{i}\right) \in M_{n \times n}\left(W\left(R_{\bar{f}_{i}}\right)\right)$. By this can be checked at every point of $R_{\bar{f}_{i}}$. This proves the first statement.

To show that $\mathcal{E}_{2} / \mathcal{E}_{1}$ is locally free, first note that for any ring homomorphism $R \rightarrow R^{\prime}$,

$$
\left(\mathcal{E}_{2} / \mathcal{E}_{1}\right) \otimes_{R} R^{\prime} \simeq\left(\mathcal{E}_{2} \otimes_{W(R)} W\left(R^{\prime}\right)\right) /\left(\mathcal{E}_{1} \otimes_{W(R)} W\left(R^{\prime}\right)\right) .
$$

So we can assume that both $E_{1}, E_{2}$ are free, and the dimension of the fibers of $E_{2} / E_{1}$ is constant $i$. Note that $E_{1} / E_{2}=\operatorname{coker}\left(E_{1} / p \rightarrow E_{2} / p\right)$. So we reduce to show that on a reduced affine scheme $\operatorname{Spec} R$, if $N$ is finitely presented $R$-module, and the fiber dimension of $N$ is constant, then $N$ is locally free. But as $N$ is finitely presented, it is the pull back

\footnotetext{
${ }^{10}$ Usually, if $\beta$ extends to a map $E_{1} \rightarrow E_{2}$, which is equivalent to requiring $m_{n} \geq 0, \beta$ then is called an isogeny. The relative position of $\beta$ is also called the Hodge polygon, and sometimes is also denoted by $H P(\beta)$. We will stick to our terminology since we do not require $E_{1} \rightarrow E_{2}$ to be regular.
} 
from some $\operatorname{coker}\left(A^{m} \rightarrow A^{n}\right)$, where $A \subset R$ is a subring, and is of finite type over $\mathbb{Z}$. Then we reduce to the noetherian situation, in which case the statement is well-known.

Recall the following fact ([Ka, $\S 2.3])$.

Lemma 1.7. $(\operatorname{Spec} R)_{\leq \lambda}$ is closed in $\operatorname{Spec} R$, and $(\operatorname{Spec} R)_{\lambda}$ is open in $(\operatorname{Spec} R)_{\leq \lambda}$. In particular, $(\operatorname{Spec} R)_{\underline{0}}$ is closed.

Here is a direct corollary of this lemma. See $\S$ A.1.4 for the definition of closed embedding between two perfect spaces.

Corollary 1.8. The diagonal map $\mathrm{Gr} \rightarrow \mathrm{Gr} \times \mathrm{Gr}$ is a closed embedding.

Proof. Let $\operatorname{Spec} R \rightarrow \operatorname{Gr} \times \operatorname{Gr}$ be a map, which are given by $(\mathcal{E}, \beta)$ and $\left(\mathcal{E}^{\prime}, \beta^{\prime}\right)$. Then we have $\left(\beta^{\prime}\right)^{-1} \beta: \mathcal{E} \rightarrow \mathcal{E}^{\prime}$. And the pullback of $R$ along the diagonal is represented by $(\operatorname{Spec} R)_{\underline{0}}$.

For every $\mu$, we define

$$
\operatorname{Gr}_{\leq \mu}(R)=\left\{(\mathcal{E}, \beta) \in \operatorname{Gr}(R) \mid(\operatorname{Spec} R)_{\leq \mu}=\operatorname{Spec} R\right\} .
$$

If $\lambda \leq \mu$, we have a closed embedding $\mathrm{Gr}_{\leq \lambda} \subset \mathrm{Gr}_{\leq \mu}$, by Lemma 1.7 We write

$$
\mathrm{Gr}_{\mu}=\mathrm{Gr}_{\leq \mu}-\cup_{\lambda<\mu} \mathrm{Gr}_{\leq \lambda} .
$$

This is an open subset of $\mathrm{Gr}_{\leq \mu}$.

For $\mu=\left(m_{1}, \ldots, m_{n}\right) \in \mathbb{X}_{\bullet}\left(D_{n}\right)^{+}$, we denote by $p^{\mu} \in \operatorname{Gr}(k)$ the lattice $\Lambda_{\mu} \subset W(k)[1 / p]^{n}$ generated by $\left\{p^{m_{1}} e_{1}, \ldots, p^{m_{n}} e_{n}\right\}$. The following lemma is the reformulation of the Cartan decomposition.

Lemma 1.9. (1) The group $\mathrm{GL}_{n}(\mathcal{O})$ acts transitively on $\mathrm{Gr}_{\mu}(k)$ and $\mathrm{Gr}_{\mu}(k)=\mathrm{GL}_{n}(\mathcal{O}) p^{\mu}$. (2) $\operatorname{Gr}(k)=\sqcup_{\lambda \in \mathbb{X} \bullet\left(D_{n}\right)^{+}} \operatorname{Gr}_{\lambda}(k)$.

We write $\overline{\mathrm{Gr}}_{N}$ instead of $\mathrm{Gr}_{\leq N \omega_{1}}$, and $\mathrm{Gr}_{N}$ instead of $\mathrm{Gr}_{N \omega_{1}}$. Note that it is in fact enough to prove the representability of $\overline{\mathrm{Gr}}_{N}$. Indeed, the group $L \mathrm{GL}_{n}$ acts on $\mathrm{Gr}$, and every $\mathrm{Gr}_{\leq \lambda}$ is contained in $g \overline{\mathrm{Gr}}_{N}$ for some $g \in \mathrm{GL}_{n}(F)$ and some $N$ and $\mathrm{Gr}_{\leq \lambda} \subset g \overline{\mathrm{Gr}}_{N}$ is a closed embedding.

1.3. Representability. In this subsection, we prove that $\overline{\mathrm{Gr}}_{N}$ is representable, and therefore $\mathrm{Gr}$ is representable. Recall the definition

$$
\overline{\operatorname{Gr}}_{N}(R)=\left\{\begin{array}{l|l}
(\mathcal{E}, \beta) & \begin{array}{l}
\mathcal{E} \text { is projective } W(R) \text {-module of rank } n, \beta: \mathcal{E} \rightarrow \mathcal{E}_{0}, \\
\text { such that } \wedge^{n} \beta: \wedge^{n} \mathcal{E} \simeq p^{N} W(R) \subset \wedge^{n} \mathcal{E}_{0}
\end{array}
\end{array}\right\} .
$$

Let $M_{n}$ denote the scheme of $n \times n$ matrices. We consider the map

$$
\pi: M:=M_{n} \times \mathbb{G}_{m} \rightarrow \mathbb{A}^{1}, \quad \pi(A, t)=t \operatorname{det} A,
$$

defined over $\mathbb{Z}_{p}$. We consider $i_{N}: \operatorname{Spec} \mathbb{Z}_{p} \rightarrow \mathbb{A}_{\mathbb{Z}_{p}}^{1}=\operatorname{Spec} \mathbb{Z}_{p}[u], u \mapsto p^{N}$, and let

$$
V_{N}=i_{N}^{*} M
$$

which is a scheme of finite type over $\mathbb{Z}_{p}$. By definition, $L_{p}^{+} V_{N}(R)$ is the set of $n \times n$-matrices $A$ with entries in $W(R)$ and $t \in W(R)^{\times}$such that $t \operatorname{det} A=p^{N}$. Note that $L_{p}^{+} \mathrm{GL}_{n}$ acts on $L_{p}^{+} V_{N}$ by left and right multiplications. Passing to perfection, both actions are free.

Lemma 1.10. There is a canonical isomorphism

$$
L^{+} V_{N} / L^{+} \mathrm{GL}_{n}=\overline{\mathrm{Gr}}_{N} .
$$


Proof. The claim is clear if we interpret $L^{+} V_{N}$ as the following moduli functor: for a perfect $k$-algebra $R$,

$$
L^{+} V_{N}(R) \simeq\left\{\begin{array}{l|l}
(\mathcal{E}, \beta, \epsilon) & \begin{array}{l}
(\mathcal{E}, \beta) \in \overline{\mathrm{Gr}}_{N}(R) \\
\epsilon: \mathcal{E}_{0} \simeq \mathcal{E} \text { is a trivialization }
\end{array}
\end{array}\right\}
$$

In fact, the above isomorphism given by $A \mapsto\left(\mathcal{E}_{0}, A\right.$, id $)$ with the inverse map given by $(\mathcal{E}, \beta, \epsilon) \mapsto A:=\beta \epsilon$.

The above lemma expresses $\overline{\mathrm{Gr}}_{N}$ as a quotient of an affine scheme by an affine group scheme. But this is not very useful as both $L^{+} V_{N}$ and $L^{+} \mathrm{GL}_{n}$ are infinite dimensional. We need to work at the finite level.

Recall that we have the affine group scheme $L^{+} \mathrm{GL}_{n}^{(h)}=\operatorname{ker}\left(L^{+} \mathrm{GL}_{n} \rightarrow L^{h} \mathrm{GL}_{n}\right)$. Let

$$
\overline{\mathrm{Gr}}_{N, h}=L^{+} V_{N} / L^{+} \mathrm{GL}_{n}^{(h)} \text {. }
$$

In terms of moduli interpretation,

$$
\overline{\operatorname{Gr}}_{N, h}(R)=\left\{\begin{array}{l|l}
(\mathcal{E}, \beta, \bar{\epsilon}) & \begin{array}{l}
(\mathcal{E}, \beta) \in \overline{\operatorname{Gr}}_{N}(R) \\
\bar{\epsilon}:\left.\left.\mathcal{E}_{0}\right|_{W_{h}(R)} \simeq \mathcal{E}\right|_{W_{h}(R)}
\end{array}
\end{array}\right\} .
$$

This is an $L^{h} \mathrm{GL}_{n}$-torsor over $\overline{\mathrm{Gr}}_{N}$ on which $L^{h} \mathrm{GL}_{n}$ acts by changing the trivialization $\bar{\epsilon}$. Our main observation is that $\overline{\mathrm{Gr}}_{N, h}$ is already represented by an affine scheme when $h$ is large. To prove this, we need to introduce certain affine schemes defined by matrix equations.

We assume that $h>N$. Via the Greenberg realization, the determinant map det: $M_{n} \rightarrow$ $\mathbb{A}^{1}$ gives rise to

$$
\left(\operatorname{det}_{0}, \ldots, \operatorname{det}_{h-1}\right): L_{p}^{h} M_{n}=\mathbb{A}^{n^{2} h} \rightarrow L_{p}^{h} \mathbb{A}^{1}=\mathbb{A}^{h}
$$

Let

$$
V_{N, h}^{\prime}:=\left\{A \in L_{p}^{h} M_{n} \mid \operatorname{det}_{0} A=\cdots=\operatorname{det}_{N-1} A=0, \operatorname{det}_{N} A \in \mathbb{G}_{m}\right\}, \quad V_{N, h}:=\left(V_{N, h}^{\prime}\right)^{p^{-\infty}} .
$$

Note that there is an $L_{p}^{h} \mathrm{GL}_{n} \times L_{p}^{h} \mathrm{GL}_{n}$-action on $V_{N, h}^{\prime}$ by left and right multiplications. Passing to the perfection, we obtain the action of $L^{h} \mathrm{GL}_{n} \times L^{h} \mathrm{GL}_{n}$ on $V_{N, h}$. Let $J$ be the stabilizer group scheme over $V_{N, h}$ with respect to the right multiplication by $L^{h} \mathrm{GL}_{n}$, i.e. $J$ is defined by the Cartesian production

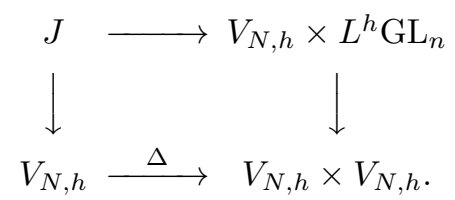

Or explicitly

$$
J=\left\{(A, \gamma) \in V_{N, h} \times L^{h} \mathrm{GL}_{n} \mid A \gamma=\gamma\right\} .
$$

Likewise, let $J^{\prime}$ denote the stabilizer group scheme over $V_{N, h}^{\prime}$ with respect to the right multiplication by $L_{p}^{h} \mathrm{GL}_{n}$. Then $J^{\prime}$ is an affine scheme of finite type over $k$, which is a deperfection of $J$.

There is a natural map

$$
\overline{\mathrm{Gr}}_{N, h} \rightarrow V_{N, h},
$$

given by $(\mathcal{E}, \beta, \bar{\epsilon}) \mapsto\left(\left.\beta\right|_{W_{h}(R)}\right) \bar{\epsilon}$.

The key lemma is the following. Recall the assumption that $h>N$.

Lemma 1.11. There is an isomorphism

$$
J \simeq \overline{\mathrm{Gr}}_{N, h} .
$$

In particular, $\overline{\mathrm{Gr}}_{N, h}$ is represented by a perfect affine scheme, perfectly of finite type. 
Proof. Recall that $J=\left(J^{\prime}\right)^{p^{-\infty}}$. Therefore the second statement follows from the first, which we now prove.

Let $R$ be a perfect $k$-algebra, and $A$ is an $R$-point of $V_{N, h}$. Then $J_{R}$ classifies those $\gamma$ making the following diagram commute



On the other hand, $\left(\overline{\mathrm{Gr}}_{N, h}\right)_{R}$ classifies the following diagram

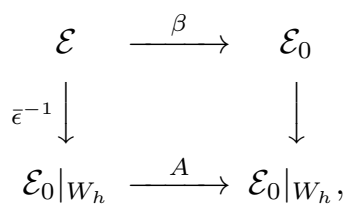

where the notation $\bar{\epsilon}^{-1}$ is understood as $\left.\left.\mathcal{E} \rightarrow \mathcal{E}\right|_{W_{h}(R)} \stackrel{\bar{\epsilon}^{-1}}{\rightarrow} \mathcal{E}_{0}\right|_{W_{h}(R)}$. Therefore, there is a natural action

$$
\overline{\mathrm{Gr}}_{N, h} \times_{V_{N, h}} J \rightarrow \overline{\mathrm{Gr}}_{N, h}, \quad((\mathcal{E}, \beta, \epsilon), \gamma) \mapsto(\mathcal{E}, \beta, \epsilon \gamma) .
$$

Note that the natural map $L^{+} V_{N} \rightarrow V_{N, h}$ is surjective on $R$-points if $h>N$. Indeed, if $A \in V_{N, h}(R)$, then $\operatorname{det} A \in p^{N} W_{h}(R)^{\times}$. Regard $A$ as a matrix in $M_{n}\left(W_{h}(R)\right)$, and let $\tilde{A} \in M_{n}(W(R))$ be any of its lift. Then $\operatorname{det} \tilde{A} \in p^{N} W(R)^{\times}$, and there is a unique $t \in W(R)^{\times}$ such that $t \operatorname{det} \tilde{A}=p^{N}$. Then $(\tilde{A}, t) \in L^{+} V_{N}(R)$ that lifts $A$.

As a consequence, the map $\overline{\mathrm{Gr}}_{N, h} \rightarrow V_{N, h}$ admits a section. Indeed, if $(\tilde{A}, t) \in L^{+} V_{N}$ is a lift of $A$, then $\left(\mathcal{E}_{0}, \tilde{A}\right.$, id $) \in \overline{\mathrm{Gr}}_{N, h}$.

Let us fix such a section $s: V_{N, h} \rightarrow \overline{\mathrm{Gr}}_{N, h}, A \mapsto\left(\mathcal{E}_{A}, \beta_{A}, \bar{\epsilon}_{A}\right)$ and therefore, we have a map $s: J \rightarrow \overline{\mathrm{Gr}}_{N, h}$ for every $A \in V_{N, h}$. It is clear that this map is injective since the action of $L^{h} \mathrm{GL}_{n}$ on $\overline{\mathrm{Gr}}_{N, h}$ is free. To see it is surjective, let $(\mathcal{E}, \beta, \bar{\epsilon})$ be a point of $\overline{\mathrm{Gr}}_{N, h}$ so that $\left(\left.\beta\right|_{W_{h}(R)}\right) \epsilon=A$. Then there exists a unique $\alpha: \mathcal{E}_{A} \simeq \mathcal{E}$ such that the following diagram commutes

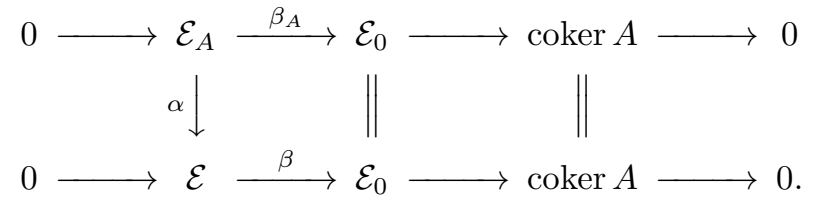

Let $\gamma=\bar{\epsilon}_{A}\left(\left.\alpha\right|_{W_{h}(R)}\right)^{-1} \bar{\epsilon}^{-1}$. Then $(A, \gamma) \in J$ is the preimage of $(\mathcal{E}, \beta, \bar{\epsilon})$ under the above map $s: J \rightarrow \overline{\mathrm{Gr}}_{N, h}$. Therefore the first claim of the lemma follows.

Remark 1.12. The above isomorphism depends on a choice of lifting of the projection $L^{+} V_{N} \rightarrow V_{N, h}$. To fix the idea, we will use the obvious lifting given by

$$
W_{h}(R) \rightarrow W(R), \quad\left(\sum_{0 \leq i<h} p^{i}\left[r_{i}\right] \quad \bmod p^{h}\right) \mapsto \sum_{0 \leq i<h} p^{i}\left[r_{i}\right] .
$$

As a corollary of the above lemma and Proposition A.28, we have

Proposition 1.13. $\overline{\mathrm{Gr}}_{N}$ is represented by a separated perfect algebraic space, perfectly of finite type over $k$.

Proof. Let $G=L^{h} \mathrm{GL}_{n}$, which is the perfection of the smooth algebraic group $G_{0}=L_{p}^{h} \mathrm{GL}_{n}$. To apply Proposition A.28, it remains to check that $G \times \overline{\mathrm{Gr}}_{N, h} \rightarrow \overline{\mathrm{Gr}}_{N, h} \times \overline{\mathrm{Gr}}_{N, h}$ is a closed embedding. But this follows from Corollary 1.8 . 
1.4. "Demazure resolution". The perfect algebraic space $\overline{\mathrm{Gr}}_{N}$ is in general "singular". We construction $\widetilde{\mathrm{Gr}}_{N} \rightarrow \overline{\mathrm{Gr}}_{N}$, which is an analogue of the Demazure resolution in the current setting. Using this map, we show that $\overline{\mathrm{Gr}}_{N}$ is irreducible and is perfectly proper.

1.4.1. Let us fix a sequence of dominant coweights of $\mu_{\bullet}=\left(\mu_{1}, \ldots, \mu_{N}\right)$ of $\mathrm{GL}_{n}$, where each $\mu_{i} \in\left\{\omega_{1}, \ldots, \omega_{n}, \omega_{1}^{*}, \ldots, \omega_{n}^{*}\right\}$.

Let us fix the free $W(k)$-module $E_{0}$ of rank $n$. We consider the following space $\widetilde{\mathrm{Gr}}_{\mu}$ on $\operatorname{Aff}_{k}^{\text {pf }}: \operatorname{Gr}_{\mu \bullet}(R)$ is the groupoid of $\left(\mathcal{E}_{N} \stackrel{\beta_{N}}{\rightarrow} \mathcal{E}_{N-1} \rightarrow \cdots \rightarrow \mathcal{E}_{1} \stackrel{\beta_{1}}{\rightarrow} \mathcal{E}_{0}=p^{*} E_{0}\right)$, where $\mathcal{E}_{i}$ is locally free of rank $n$ on $W(R), \mathcal{E}_{i} \rightarrow \mathcal{E}_{i-1}$ is a quasi-isogeny with relative position $\mu_{i}$. In particular, if $\mu_{i}=\omega_{1}$ for all $i$, we denote $\operatorname{Gr}_{\mu}$. by $\widetilde{\mathrm{Gr}}_{N}$, which classifies $\left(\mathcal{E}_{N} \rightarrow \mathcal{E}_{N-1} \rightarrow\right.$ $\cdots \rightarrow \mathcal{E}_{1} \rightarrow \mathcal{E}_{0}=p^{*} E_{0}$ ), where $\mathcal{E}_{i} \rightarrow \mathcal{E}_{i-1}$ is injective an $\mathcal{E}_{i-1} / \mathcal{E}_{i}$ is invertible on $W(R) / p$. The natural map $\widetilde{\mathrm{Gr}}_{N} \rightarrow \overline{\mathrm{Gr}}_{N}$ is given by forgetting $\left(\mathcal{E}_{N-1}, \cdots, \mathcal{E}_{1}\right)$.

Proposition 1.14. $\mathrm{Gr}_{\mu}$ is represented by a perfect $k$-scheme, perfectly proper over $k$.

Proof. We will prove this by induction on $N$. First, we show $\operatorname{Gr}_{\omega_{i}} \simeq \operatorname{Gr}^{p^{-\infty}}(i, n)$. We do it slightly more general.

Lemma 1.15. Let $X$ be a perfect $k$-scheme and $\mathcal{E}_{0}$ is a locally free crystal of rank $n$ on $X$. Let $Y$ be the perfect space over $X$ that assigns to every $\operatorname{Spec} R \rightarrow X$ the set of isogenies $\mathcal{E}_{1} \rightarrow \mathcal{E}_{0}$ of finite projective $W(R)$-modules of rank n such that $\mathcal{E}_{0} / \mathcal{E}_{1}$ are locally free $W(R) / p$ modules of rank $i$. Then $Y$ is represented by the perfect scheme $\mathrm{Gr}^{p^{-\infty}}\left(i, \mathcal{E}_{0} / p\right)$ introduced in Corollary A.22.

The map $Y \rightarrow \operatorname{Gr}^{p^{-\infty}}\left(i, \mathcal{E}_{0} / p\right)$ sends $\mathcal{E}_{1} \rightarrow \mathcal{E}_{0}$ to $\mathcal{E}_{0} / p \mathcal{E}_{0} \rightarrow \mathcal{E}_{0} / \mathcal{E}_{1} \rightarrow 0$. Conversely, given $\mathcal{E}_{0} / p \mathcal{E}_{0} \rightarrow \mathcal{Q} \rightarrow 0$, we define $\mathcal{E}_{1}=\operatorname{ker}\left(\mathcal{E}_{0} \rightarrow \mathcal{E}_{0} / p \mathcal{E}_{0} \rightarrow \mathcal{Q}\right)$. We need to show this is a finite projective $W(R)$-modules of rank $n$. It is enough to show that $\mathcal{E}_{1} / p^{h} \mathcal{E}_{1}$ is finite projective $W(R) / p^{h}$-module of rank $n$ for every $h$.

We have

$$
p \mathcal{E}_{0} \subset \mathcal{E}_{1} \subset \mathcal{E}_{0} .
$$

and $\mathcal{E}_{1} / p \mathcal{E}_{0}$ is locally direct summand of $\mathcal{E}_{0} / p \mathcal{E}_{0}$, and therefore is locally free. Now, from

$$
0 \rightarrow \operatorname{Tor}^{W(R)}(\mathcal{Q}, W(R) / p) \rightarrow \mathcal{E}_{1} / p \mathcal{E}_{1} \rightarrow \mathcal{E}_{0} / p \mathcal{E}_{0} \rightarrow \mathcal{Q} \rightarrow 0,
$$

and the canonically isomorphism

$$
\mathcal{Q}=\operatorname{Tor}^{W(R)}(\mathcal{Q}, W(R) / p),
$$

we see that $\mathcal{E}_{1} / p \mathcal{E}_{1}$, which is an extension of $\mathcal{E}_{1} / p \mathcal{E}_{0}$ by $\mathcal{Q}$, is a locally free $R$-module of rank $n$. By

$$
0 \rightarrow p \mathcal{E}_{0} / p \mathcal{E}_{1} \rightarrow \mathcal{E}_{1} / p \mathcal{E}_{1} \rightarrow \mathcal{E}_{1} / p \mathcal{E}_{0} \rightarrow 0
$$

$p \mathcal{E}_{0} / p \mathcal{E}_{1}$ is locally a direct summand of $\mathcal{E}_{1} / p \mathcal{E}_{1}$, and therefore is locally free as $R$-modules. By inductions, we see each $p^{i} \mathcal{E}_{1} / p^{i+1} \mathcal{E}_{1}$ is a locally free $R$-module of rank $n$, and $p^{i+1} \mathcal{E}_{0} / p^{i+1} \mathcal{E}_{1}$ is a locally direct summand of $p^{i} \mathcal{E}_{1} / p^{i+1} \mathcal{E}_{1}$.

Using $0 \rightarrow p^{i} \mathcal{E}_{1} / p^{i+1} \mathcal{E}_{1} \rightarrow \mathcal{E}_{1} / p^{i+1} \mathcal{E}_{1} \rightarrow \mathcal{E}_{1} / p^{i} \mathcal{E}_{1} \rightarrow 0$, by induction again we see that each $\mathcal{E}_{1} / p^{i} \mathcal{E}_{1}$ is locally free.

This finishes the proof of the lemma and therefore the representability of $\mathrm{Gr}_{\omega_{i}}$. Now assume that $\mathrm{Gr}_{\mu}$ is representable by an essentially projective perfect $k$-scheme. Let $\mu_{N+1}$ be the additional coweight. Let $U=\operatorname{Spec} R$ be an affine open of $\operatorname{Gr}_{\mu_{\bullet}}$. Then by definition, over $W(R)$, there is the chain of isogenies $\mathcal{E}_{N} \rightarrow \mathcal{E}_{N-1} \rightarrow \cdots \rightarrow \mathcal{E}_{0}$, and $\mathcal{E}_{N} / p$ is a locally free sheaf of rank $n$ on $U$. Clearly, varying $U$, we get a vector bundle $\mathcal{E}_{N} / p$ on $\mathrm{Gr}_{\mu_{\bullet}}$. Using Lemma 1.15 again, $\operatorname{Gr}_{\mu_{\bullet}, \mu_{N+1}} \simeq \operatorname{Gr}^{p^{-\infty}}\left(i, \mathcal{E}_{N} / p\right)$ if $\mu_{N+1}=\omega_{i}$ or $\operatorname{Gr}_{\mu_{\bullet}, \mu_{N+1}} \simeq \operatorname{Gr}^{p^{-\infty}}\left(i,\left(\mathcal{E}_{N} / p\right)^{*}\right)$ if $\mu_{N+1}=\omega_{i}^{*}$.

By Corollary A.22, $\mathrm{Gr}_{\mu}$ is perfectly proper. 
Remark 1.16. One can show that

$$
\widetilde{\mathrm{Gr}}_{1}=\mathbb{P}^{n-1, p^{-\infty}}, \quad{\widetilde{\mathrm{Gr}_{2}}}=\mathbb{P}^{p^{-\infty}}\left(\Omega_{\mathbb{P}^{n-1}} \oplus \mathcal{O}_{\mathbb{P}^{n-1}}\right) .
$$

See $₫$ B.3 for a sample calculation. On the other hand one can define the equal characteristic Demazure variety $\widetilde{\mathrm{Gr}}_{N}^{b}$ which assigns every $k$-algebra $R$ the set of chains $\left\{\mathcal{E}_{N} \rightarrow \mathcal{E}_{N-1} \rightarrow\right.$ $\left.\cdots \rightarrow \mathcal{E}_{0}=R[[t]]^{n}\right\}$ of finite projective $R[[t]]$-modules of rank $n$ such that each $\mathcal{E}_{i} / \mathcal{E}_{i+1}$ is an invertible $R[[t]] / t$-module. Then

$$
\widetilde{\mathrm{Gr}}_{N}=\left(\widetilde{\mathrm{Gr}}_{N}^{b}\right)^{p^{-\infty}}, \quad N=1,2
$$

We do not think that this is true for general $N$.

Likewise, one can define the equal characteristic analogue $\overline{\mathrm{Gr}}_{N}^{b}$ of $\overline{\mathrm{Gr}}_{N}$ as the moduli space of pairs $(\mathcal{E}, \beta)$ where $\mathcal{E}$ is a finite projective $R[[t]]$-module of rank $n$ and $\beta: \mathcal{E} \rightarrow \mathcal{E}_{0}$ is a morphism such that $\wedge^{n} \beta=t^{N} \lambda$ for some $\lambda \in R[[t]]^{\times}$. From the example given in $₫$ B.3. when $n=2$ and $N=2, \overline{\mathrm{Gr}}_{2} \simeq\left(\overline{\mathrm{Gr}}_{2}^{b}\right)^{p^{-\infty}}$. But we do not think that this is true in general.

Let $\left|\mu_{\bullet}\right|=\sum \mu_{i}$. There is a natural map $\operatorname{Gr}_{\mu_{\bullet}} \rightarrow \operatorname{Gr}_{\leq\left|\mu_{\bullet}\right|}$ by $\left(\mathcal{E}_{\bullet}, \beta_{\bullet}\right) \mapsto\left(\mathcal{E}_{N}, \beta_{1} \cdots \beta_{N}\right)$. Similarly, we can show

Lemma 1.17. The map $\pi: \mathrm{Gr}_{\mu_{\bullet}} \rightarrow \mathrm{Gr}_{\leq\left|\mu_{\bullet}\right|}$ is representable. It is perfectly proper, and fibers are perfectly proper perfect schemes.

Proof. Let $\operatorname{Spec} R \rightarrow \operatorname{Gr}_{\leq\left|\mu_{\bullet}\right|}$ be a morphism given by $(\mathcal{E}, \beta)$. Then $\left(\operatorname{Gr}_{\mu_{\bullet}}\right)_{R}$ and all possible chain of maps $\mathcal{E}=\mathcal{E}_{N} \rightarrow \mathcal{E}_{N-1} \rightarrow \cdots \rightarrow \mathcal{E}_{0}$ such that their composition is $\beta$, and $\operatorname{inv}\left(\mathcal{E}_{i} \rightarrow\right.$ $\left.\mathcal{E}_{i-1}\right)=\mu_{i}$. We consider the moduli problem $X$ such that for every $R \rightarrow R^{\prime}$

$$
X\left(R^{\prime}\right)=\left\{\mathcal{F}_{N} \leftarrow \mathcal{F}_{N-1} \leftarrow \cdots \leftarrow \mathcal{F}_{0}=\mathcal{E} \mid \operatorname{inv}\left(\mathcal{F}_{i}, \mathcal{F}_{i+1}\right)=\mu_{i}^{*}\right\} .
$$

By Lemma 1.15, $X$ is represented by a perfect scheme over $R$, perfectly proper over $R$. Over $X$ we consider the quasi-isogeny $\mathcal{F}_{N} \rightarrow \mathcal{F}_{0}=\mathcal{E} \rightarrow \mathcal{E}_{0}$. Then $\left(\operatorname{Gr}_{\mu_{\bullet}}\right)_{R}$ is just represented by $X_{\underline{0}}$, which is closed in $X$ by Lemma 1.7

Lemma 1.18. Then the restriction of $\pi: \widetilde{G r}_{N} \rightarrow \overline{\mathrm{Gr}}_{N}$ to $\mathrm{Gr}_{N}$ is an isomorphism. The fiber over any $x \in \overline{\mathrm{Gr}}_{N}-\mathrm{Gr}_{N}$ is non-empty, geometrically connected, and has positive dimension.

Proof. It is clear that every $E \rightarrow E_{0}$ over $W(k)$ can be factors as $E=E_{N} \rightarrow E_{N-1} \rightarrow$ $\cdots \rightarrow E_{1} \rightarrow E_{0}$ such that $E_{i} / E_{i+1}$ is a one-dimensional vector space over $k$. This proves that the fibers of $\pi$ are non-empty.

Next, we show that $\pi: \pi^{-1}\left(\operatorname{Gr}_{N}\right) \rightarrow \operatorname{Gr}_{N}$ is an isomorphism by exhibiting an inverse morphism. Indeed, given such $(\mathcal{E}, \beta) \in \operatorname{Gr}_{N}(R)$, there is a filtration of finitely generated $W(R)$-modules

$$
\mathcal{E}=\mathcal{E}_{N} \subset \mathcal{E}_{N-1} \subset \cdots \subset \mathcal{E}_{0}=\mathcal{E}_{0}
$$

with $\mathcal{E}_{i}=\mathcal{E}+p^{i} \mathcal{E}_{0}$. It is enough to show that each $\mathcal{E}_{i}$ is a projective $W(R)$-modules and $\mathcal{E}_{i} / \mathcal{E}_{i+1}$ is an invertible $W(R) / p$-module. Indeed, at each point $x \in \operatorname{Spec} R$, the dimension of the stalk of $\mathcal{E}_{i} / \mathcal{E}_{i+1}$ is one. Then by the end of the proof of Lemma 1.6, $\mathcal{E}_{i} / \mathcal{E}_{i+1}$ is invertible. And by the same argument of Lemma 1.15] and by induction of $i$, each $\mathcal{E}_{i}$ is a projective $W(R)$-module.

Next we show that the fibers over $x \in \overline{\mathrm{Gr}}_{N}-\mathrm{Gr}_{N}$ has positive dimension. First note that $\widetilde{\mathrm{Gr}}_{N} \rightarrow \overline{\mathrm{Gr}}_{N}$ is $L^{+} \mathrm{GL}_{n}$-equivariant, where $L^{+} \mathrm{GL}_{n}$ acts via automorphisms of $E_{0}$. By Lemma 1.9, it is enough to show that the fibers over $p^{\lambda} \in \overline{\operatorname{Gr}}_{N}(k)$ for $\lambda<N \omega_{1}$ has positive dimension, where $p^{\lambda} \in \operatorname{Gr}(k)$ is the point introduced before Lemma 1.9. But if $\lambda<N \omega_{1}$, there exists some $i$ such that $\operatorname{dim}_{k}\left(E_{\lambda} \cap p^{i} E_{0} / E_{\lambda} \cap p^{i+1} E_{0}\right)>1$. Therefore the fiber contains at least a $\mathbb{P}^{1, p^{-\infty}}$, corresponding to possible choices of a line in $\left(E_{\lambda} \cap p^{i} E_{0} / E_{\lambda} \cap p^{i+1} E_{0}\right)$.

Finally, we show that the fibers are geometrically connected. We factor $\widetilde{\mathrm{Gr}}_{N} \rightarrow \overline{\mathrm{Gr}}_{N}$ as

$$
{\widetilde{\mathrm{Gr}_{N}}}_{N} \stackrel{\pi_{1}}{\rightarrow} \mathbb{P}^{p^{-\infty}}(\mathcal{E} / p) \stackrel{\pi_{2}}{\rightarrow} \overline{\mathrm{Gr}}_{N}
$$


where $\mathcal{E} \rightarrow \mathcal{E}_{0}$ is the universal family over $\overline{\mathrm{Gr}}_{N-1}$. Given $\left(\mathcal{E}_{\bullet}, \beta_{\bullet}\right) \in \widetilde{\mathrm{Gr}}_{\mu_{\bullet}}$, the first map forgets $\mathcal{E}_{N-2}, \ldots, \mathcal{E}_{1}$, and the second map further forgets $\mathcal{E}_{N-1}$. By induction, the first map has geometrically connected fiber. And it is easy to see that given $(\mathcal{E}, \beta) \in \overline{\mathrm{Gr}}_{N}$, the fiber of $\pi_{2}$ over this point is given by $\mathbb{P}^{p^{-\infty}}\left(\left(p^{-1} \mathcal{E} \cap \mathcal{E}_{0}\right) / \mathcal{E}\right)$, which is the perfection of a projective space. Together, it shows that the fibers of $\pi$ are geometrically connected.

We have the following consequence.

Corollary 1.19. The algebraic space $\overline{\mathrm{Gr}}_{N}$ is irreducible, and is perfectly proper.

\subsection{The affine Grassmannian and affine flag variety.}

1.5.1. Once the representability of $\mathrm{Gr}_{\mathrm{GL}_{n}}$ is established, it is not hard to show that for a general smooth affine group scheme $\mathcal{G}$ over $\mathcal{O}$, the corresponding affine Grassmannian $\mathrm{Gr}_{\mathcal{G}}=L \mathcal{G} / L^{+} \mathcal{G}$ is representable.

Proposition 1.20. Let $\rho: \mathcal{G} \rightarrow \mathrm{GL}_{n}$ be a linear representation such that $\mathrm{GL}_{n} / \mathcal{G}$ is quasiaffine, then $\mathrm{Gr}_{\mathcal{G}} \rightarrow \mathrm{Gr}_{\mathrm{GL}_{n}}$ is a locally closed embedding. In addition, if $\mathrm{GL}_{n} / \mathcal{G}$ is affine, this is a closed embedding.

Proof. The proof as in [BD, Theorem 4.5.1] or [PR, Theorem 1.4] extends verbatim to the present situation.

For a smooth affine group scheme $\mathcal{G}$ over a Dedekind domain, it is well-known that there is always a linear representation $\rho: \mathcal{G} \rightarrow \mathrm{GL}_{n}$ such that $\mathrm{GL}_{n} / \mathcal{G}$ is quasi-affine (cf. PR, $\S 1 . b]$ ). In addition, if $\mathcal{G}=G$ is reductive, then one can choose $\rho$ such that $\mathrm{GL}_{n} / G$ is affine. Therefore, this proposition completes the proof of Theorem 1.5. It in fact gives more. Namely, let $\mathcal{G}$ be a parahoric group scheme of $G$ in the sense of Bruhat-Tits, then $\operatorname{Gr}_{\mathcal{G}}$ is still representable. We will see, in fact, that $\mathrm{Gr}_{\mathcal{G}}$ is ind perfectly proper.

1.5.2. As the theory is completely parallel to the equal characteristic situation (after passing to the perfection), we will be sketchy here. We assume that $k$ is algebraically closed. Let $G$ be a reductive group over $F$. Let $B(G, F)$ denote the Bruhat-Tits building of $G$. We fix an apartment $A(G, F) \subset B(G, F)$ and an alcove $\mathbf{a} \subset A(G, F)$. This determines an Iwahori group scheme $\mathcal{G}_{C}$ of $G$ over $\mathcal{O}$. Let $\widetilde{W}$ denote the Iwahori-Weyl group, which is the quotient of the normalizer $N(F)$ of $T(F)$ by $T(\mathcal{O})$, and let $W_{a} \subset \widetilde{W}$ denote the affine Weyl group. Let $s_{i}, i \in \mathbb{S}$ denote the simple reflections, corresponding to the codimension one walls $\mathbf{a}_{i}$ of $\overline{\mathbf{a}}$, and let " $\leq$ " denote the Bruhat order on $\widetilde{W}$. Let $\mathcal{G}_{i}$ denote the corresponding parahoric group scheme. There is a natural map $\mathcal{G}_{C} \rightarrow \mathcal{G}_{i}$. Let $I=L^{+} \mathcal{G}_{\mathrm{a}}$ and $P_{i}=L^{+} \mathcal{G}_{i}$. Let us write $\mathcal{F} \ell=L G / I$, and call it the affine flag variety of $G$. By Proposition 1.20 it is representable. For $w \in \widetilde{W}$, let $S_{w}$ denote the closure of the $I$-orbit through $\dot{w}$, where $\dot{w}$ is a lifting of $w$ to $G(F)$. This is the "Schubert variety", which in the current setting is a separated perfect php algebraic space. As in the equal characteristic situation,

$$
S_{w}=\bigsqcup_{v \leq w} I \dot{v} I / I
$$

is a decomposition of $S_{w}$ as locally closed subsets and each $I \dot{v} I / I$ is isomorphic to the perfection of an affine space of dimension $\ell(v)$. We show that $S_{w}$ is perfectly proper so that $\mathcal{F} \ell=\underline{\lim } S_{w}$ is ind perfectly proper. The idea is similar to Corollary 1.19.

Note that $I$ is a subgroup of $P_{i}$ (however, $L_{p}^{+} \mathcal{G}_{C} \rightarrow L_{p}^{+} \mathcal{G}_{i}$ is not a closed embedding). It is easy to see that $P_{i} / I \simeq \mathbb{P}^{1, p^{-\infty}}$. Then for any sequence $\tilde{w}=\left(s_{j_{1}}, \ldots, s_{j_{m}}\right), j_{1}, \ldots, j_{m} \in \mathbb{S}$ (or called a word), one can form the "Demazure" variety

$$
D_{\tilde{w}}=P_{j_{1}} \times{ }^{I} P_{j_{2}} \times{ }^{I} \cdots \times P_{j_{m}} / I .
$$


Similar to $\widetilde{\mathrm{Gr}}_{N}$, this is an iterated $\mathbb{P}^{1, p^{-\infty}}$-bundle. In particular, it is perfectly proper. Now assume that $\tilde{w}$ is a reduced word, i.e. the length $\ell(w)=m$, where $w=s_{j_{1}} \cdots s_{j_{m}}$. Then as in $[\mathrm{PR}, \S 8]$, there is a surjective map

$$
\pi_{\tilde{w}}: D_{\tilde{w}} \rightarrow S_{w},
$$

with geometrically connected fibers. This shows that $S_{w}$ is perfectly proper. As in the equal characteristic situation,

Proposition 1.21. There is a canonical isomorphism $\pi_{1}(G)_{\operatorname{Gal}(\bar{F} / F)} \simeq \pi_{0}(L G) \simeq \pi_{0}\left(\operatorname{Gr}_{\mathcal{G}}\right)$.

Proof. One can argue as in [PR, §5]: By the standard argument (using the $z$-extension), it reduces to consider $G=T$ is a torus or $G=G_{\mathrm{sc}}$ is semisimple and simply connected. Note that the functor $\pi_{0}(L T)$ from the category of $F$-tori to the category of abelian groups satisfies the condition of [Ko, $\S 2]$, and therefore the statement of the proposition holds for $G=T$. Using the "Demazure resolution" and the Cartan decomposition, one concludes that $L G$ is connected if $G$ is simply-connected.

1.5.3. Now we concentrate on the case $G$ is a reductive group over $\mathcal{O}$. We denote the corresponding affine Grassmannian by $\operatorname{Gr}_{G}$. Let $\beta: \mathcal{E}_{1}[1 / p] \simeq \mathcal{E}_{2}[1 / p]$ be a quasi-isogeny between two $G$-torsors over $\mathcal{O}$. It makes sense to talk about the relative position $\operatorname{Inv}(\beta)$ of $\beta$, which is an element in $\mathbb{X}_{\bullet}(T)^{+}$, the set of dominant coweights of $G$. We denote the Schubert variety $\mathrm{Gr}_{\leq \mu}$ in $\mathrm{Gr}$ as

$$
\operatorname{Gr}_{\leq \mu}=\{(\mathcal{E}, \beta) \in \operatorname{Gr} \mid \operatorname{Inv}(\beta) \leq \mu\} .
$$

Here are some basic facts about the Schubert varieties. The proof is standard and is omitted.

Proposition 1.22. (1) $\mathrm{Gr}_{\mu} \subset \mathrm{Gr}_{\leq \mu}$ is open, and $\mathrm{Gr}_{\leq \lambda} \subset \mathrm{Gr}_{\leq \mu}$ if and only if $\lambda \leq \mu$.

(2) $\mathrm{Gr}_{\mu}$ is perfectly smooth of dimension $(2 \rho, \mu)$, and $\mathrm{Gr}_{\leq \mu}$ is perfectly proper of dimen$\operatorname{sion}(2 \rho, \mu)$.

Proof. (1) follows from Lemma 1.7 and the Tannakian considerations. (2) follows from that $\mathrm{Gr}_{\mu} \simeq L^{+} G / L^{+} G \cap \varpi^{\mu} L^{+} G \varpi^{-\mu}$.

There is a map $\mathbb{X}_{\bullet} \rightarrow \mathbb{Z} / 2, \mu \mapsto(-1)^{(2 \rho, \mu)}$, which factors through $\mathbb{X} \bullet(T) \rightarrow \pi_{1}(G) \rightarrow \mathbb{Z} / 2$ and therefore induces a continuous map

$$
p: \mathrm{Gr}_{G} \rightarrow \mathbb{Z} / 2
$$

by Proposition 1.21 Note that

Lemma 1.23. The Schubert cell $\mathrm{Gr}_{\mu}$ is in the even (resp. odd) components, i.e. $p\left(\mathrm{Gr}_{\mu}\right)=1$ (resp. $p\left(\mathrm{Gr}_{\mu}\right)=-1$ ) if and only if $\operatorname{dim} \mathrm{Gr}_{\mu}$ is even (resp. odd).

\section{The geometric Satake}

We establish the geometric Satake correspondence in this setting. We will assume that $k$ is algebraically closed in this section, and let $G$ denote a reductive group over $\mathcal{O}$. As explained in the introduction, one can define the category of $L^{+} G$-equivariant perverse sheaf on $\mathrm{Gr}_{G}$, denoted by $\mathrm{P}_{L^{+} G}\left(\mathrm{Gr}_{G}\right)$. As will be explained below, there is a convolution product that makes this a semisimple monoidal category. In addition, the global cohomology functor is a natural monoidal funcor. Then we establish the commutativity constraints using some combinatoric formula from the theory of affine Hecke algebra. In the course, we will also develop the Mirković-Vilonen's theory in this situation.

For simplicity, we will write $\mathrm{Gr}$ for $\mathrm{Gr}_{G}$ if the group $G$ is clear. Proofs are sketchy or omitted if they are similar to their equal characteristic counterparts.

\subsection{The category $\mathrm{P}_{L^{+}}\left(\mathrm{Gr}_{G}\right)$.}


2.1.1. As $\operatorname{Gr}_{G}$ can be written as inductive limit of $L^{+} G$-invariant closed subsets $\mathrm{Gr}_{<\mu}$, which are perfectly proper, and the action of $L^{+} G$ on $\mathrm{Gr}_{\leq \mu}$ factors through some $L^{\bar{h}} G$ which is perfectly of finite type, it makes sense to talk about the category of $L^{+} G$-equivariant perverse sheaves on $\mathrm{Gr}_{\leq \mu}$ (see $\mathrm{A.3.5}$ ), denoted by $\mathrm{P}_{L^{+} G}\left(\mathrm{Gr}_{\leq \mu}\right)$. Then we denote by

$$
\mathrm{P}_{L^{+} G}\left(\mathrm{Gr}_{G}\right)=\underset{\lim }{\longrightarrow} \mathrm{P}_{L^{+} G}\left(\mathrm{Gr}_{\leq \mu}\right)
$$

the category of $L^{+} G$-equivariant perverse sheaves on $\operatorname{Gr}_{G}$. Denote by $\mathrm{IC}_{\mu}$ the intersection cohomology sheaf on $\mathrm{Gr}_{\leq \mu}$. Then $\left.\operatorname{IC}_{\mu}\right|_{\operatorname{Gr}_{\mu}}=\overline{\mathbb{Q}}_{\ell}[(2 \rho, \mu)]((\rho, \mu))$, and its restriction to each stratum $\mathrm{Gr}_{\lambda}$ is constant. As

$$
\mathrm{Gr}_{\mu}=L^{+} G /\left(L^{+} G \cap \varpi^{\mu} L^{+} G \varpi^{-\mu}\right)
$$

and $L^{+} G \cap \varpi^{\mu} L^{+} G \varpi^{-\mu}$ is connected, the irreducible objects of $\mathrm{P}_{L^{+} G}\left(\operatorname{Gr}_{G}\right)$ are exactly these $\mathrm{IC}_{\mu}$ 's.

Lemma 2.1. The category $\mathrm{P}_{L^{+} G}\left(\mathrm{Gr}_{G}\right)$ is semisimple.

Proof. The proof is literally the same as the equal characteristic situation (see [Lu1] and Ga, Appendix] for details): The existence of the "Demzure resolution" (see (1.5.2)) and the fibers of $\pi_{\tilde{w}}$ have a paving by (perfect) affine spaces implies the parity property of the stalk cohomology of $\mathrm{IC}_{\mu} \mathrm{s}$. Together with Lemma 1.23, one concludes that there is no extension between to irreducible objects.

2.1.2. We refer to A.1.3 for the definition of twisted product, which will also be called convolution product in the current setting. Now we begin with the $L^{+} G$-torsor $L G \rightarrow \mathrm{Gr}$ and the $L^{+} G$ space Gr, we can form the convolution affine Grassmannian Gr $\tilde{x}$ Gr. As in the equal characteristic situation (e.g. $[\mathrm{MV}]$ ), one can interpret $\mathrm{Gr} \tilde{\times} \mathrm{Gr}$ as

$$
\operatorname{Gr} \tilde{\times} \operatorname{Gr}(R)=\left\{\begin{array}{l|l}
\left(\mathcal{E}_{1}, \mathcal{E}_{2}, \beta_{1}, \beta_{2}\right) & \begin{array}{l}
\mathcal{E}_{1}, \mathcal{E}_{2} \text { are } G \text {-torsors on } W(R), \\
\beta_{1}: \mathcal{E}_{1}[1 / p] \simeq \mathcal{E}_{0}[1 / p], \beta_{2}: \mathcal{E}_{2}[1 / p] \simeq \mathcal{E}_{1}[1 / p]
\end{array}
\end{array}\right\}
$$

Note that there is the convolution product map

$$
m: \operatorname{Gr} \tilde{\times} \mathrm{Gr} \rightarrow \mathrm{Gr}, \quad\left(\mathcal{E}_{1}, \mathcal{E}_{2}, \beta_{1}, \beta_{2}\right) \mapsto\left(\mathcal{E}_{2}, \beta_{1} \beta_{2}\right)
$$

and the natural projection

$$
\operatorname{pr}_{1}: \operatorname{Gr} \tilde{\times} \mathrm{Gr} \rightarrow \mathrm{Gr}, \quad\left(\mathcal{E}_{1}, \mathcal{E}_{2}, \beta_{1}, \beta_{2}\right) \mapsto\left(\mathcal{E}_{1}, \beta_{1}\right),
$$

which induces $\left(\mathrm{pr}_{1}, m\right): \mathrm{Gr} \tilde{\times} \mathrm{Gr} \simeq \mathrm{Gr} \times \mathrm{Gr}$. In particular, the convolution Grassmannian is representable as ind perfect algebraic spaces, ind perfectly proper. Given $\mu_{1}, \mu_{2} \in \mathbb{X}_{\bullet}^{+}$of $G$, we can form the convolution product of $\mathrm{Gr}_{\leq \mu_{1}}$ and $\mathrm{Gr}_{\leq \mu_{2}}$,

$$
\operatorname{Gr}_{\leq \mu_{1}} \tilde{\times} \operatorname{Gr}_{\leq \mu_{2}}=\left\{\left(\mathcal{E}_{1}, \mathcal{E}_{2}, \beta_{1}, \beta_{2}\right) \in \operatorname{Gr} \tilde{\times} \operatorname{Gr} \mid \operatorname{Inv}\left(\beta_{1}\right) \leq \mu_{1}, \operatorname{Inv}\left(\beta_{2}\right) \leq \mu_{2} .\right\},
$$

which is closed in $\operatorname{Gr} \tilde{\times} \mathrm{Gr}$ and therefore is representable. Similarly, one can form the $n$-fold convolution Grassmannian $\operatorname{Gr} \tilde{x} \cdots \tilde{x} \mathrm{Gr}$ and if $\mu_{\bullet}=\left(\mu_{1}, \ldots, \mu_{n}\right)$ is a sequence of dominant coweights of $G$, we have $\mathrm{Gr}_{\leq \mu_{\bullet}}=\mathrm{Gr}_{\leq \mu_{1}} \tilde{x} \cdots \tilde{\times} \mathrm{Gr}_{\leq \mu_{n}}$. Let $\left|\mu_{\bullet}\right|=\sum \mu_{i}$, then the convolution product is the map

$$
m: \mathrm{Gr}_{\leq \mu_{\bullet}} \rightarrow \mathrm{Gr}_{\leq\left|\mu_{\bullet}\right|}, \quad\left(\mathcal{E}_{\bullet}, \beta_{\bullet}\right) \mapsto\left(\mathcal{E}_{n}, \beta_{1} \cdots \beta_{n}\right) .
$$

There are variants of the above construction. Namely, one can replace $\mathrm{Gr}_{\leq \mu_{i}}$ by $\mathrm{Gr}_{\mu_{i}}$ and form $\mathrm{Gr}_{\mu_{\bullet}}=\mathrm{Gr}_{\mu_{1}} \tilde{\times} \cdots \tilde{\times} \mathrm{Gr}_{\mu_{n}}$. In particular,

$$
\mathrm{Gr}_{\leq \mu_{\bullet}}=\bigcup_{\mu_{\bullet}^{\prime} \leq \mu_{\bullet}} \mathrm{Gr}_{\mu_{\bullet}^{\prime}}
$$

form a stratification of $\mathrm{Gr}_{\leq \mu_{\bullet}}$, where $\mu_{\bullet}^{\prime} \leq \mu_{\bullet}$ means $\mu_{i}^{\prime} \leq \mu_{i}$ for each $i$.

Now, as in the equal characteristic situation, one can define a monoidal structure on $\mathrm{P}_{L^{+} G}(\mathrm{Gr})$, using Lusztig's convolution of sheaves (e.g. see [MV, §4] for more details). For $\mathcal{A}_{1}, \mathcal{A}_{2} \in \mathrm{P}_{L^{+} G}(\mathrm{Gr})$, we denote by $\mathcal{A}_{1} \tilde{\otimes} \mathcal{A}_{2}$ the "external twisted product" of $\mathcal{A}_{1}$ and $\mathcal{A}_{2}$ on 
Gr $\tilde{\times}$ Gr, i.e., the pullback of $\mathcal{A}_{1} \tilde{\otimes} \mathcal{A}_{2}$ along $L G \times \mathrm{Gr} \rightarrow \mathrm{Gr} \tilde{\times} \mathrm{Gr}$ is equal to the pullback the external product $\mathcal{A}_{1} \otimes \mathcal{A}_{2}$ along $L G \times \mathrm{Gr} \rightarrow \mathrm{Gr} \times \mathrm{Gr}$. Let

$$
\mathcal{A}_{1} \star \mathcal{A}_{2}:=m_{!}\left(\mathcal{A}_{1} \tilde{\otimes} \mathcal{A}_{2}\right)
$$

their convolution product, which is an $L^{+} G$-equivariant $\ell$-adic complex on Gr. Similarly, one can define the $n$-fold convolution $\mathcal{A}_{1} \star \cdots \star \mathcal{A}_{n}=m_{!}\left(\mathcal{A}_{1} \tilde{\otimes} \cdots \tilde{\otimes} \mathcal{A}_{n}\right)$.

Proposition 2.2. The convolution $\mathcal{A}_{1} \star \mathcal{A}_{2}$ is perverse.

This can be proved using the numerical results of the Hecke algebras [Lu1] (see Gi] for details). We will outline another proof in the next subsection (see \$2.2.3) following [NP, §9], after we introduce the semi-infinite orbits.

There is an equivalent formulation of this proposition.

Proposition 2.3. The convolution product $m: \mathrm{Gr}_{\leq \mu} \rightarrow \mathrm{Gr}$ is semismall. I.e., the dimension of $\operatorname{Gr}_{\leq \mu_{\bullet}}^{\lambda}:=m^{-1}\left(\mathrm{Gr}_{\leq \lambda}\right)$ is at most $\left(\rho,\left|\mu_{\bullet}\right|+\lambda\right)$.

Proof. The direction from Proposition 2.3 to Proposition 2.2 is [MV, Lemma 4.3]. The inverse direction is mentioned in [MV] Remark 4.5], without the proof. As we will make use of this statement in Proposition 3.4 we include a sketch.

Let $d=\operatorname{dim} \mathrm{Gr}_{\mu} \cap \cap m^{-1}\left(\varpi^{\lambda}\right)$. By Lemma 2.1 and Proposition 2.2, we can write

$$
\mathrm{IC}_{\mu_{\bullet}}:=\mathrm{IC}_{\mu_{1}} \star \cdots \star \mathrm{IC}_{\mu_{n}}=\bigoplus_{\lambda} V_{\mu_{\bullet}}^{\lambda} \otimes \mathrm{IC}_{\lambda}
$$

where $V_{\mu_{\bullet}}^{\lambda}=\operatorname{Hom}\left(\mathrm{IC}_{\lambda}, \mathrm{IC}_{\mu_{\bullet}}\right)$. An easy spectral sequence argument shows that the degree $2 d-\left(2 \rho,\left|\mu_{\bullet}\right|\right)$ stalk cohomology at $\varpi^{\lambda}$ of the left hand side is given

$$
\mathrm{H}_{c}^{2 d}\left(\mathrm{Gr}_{\mu_{\bullet}} \cap m^{-1}\left(\varpi^{\lambda}\right), \overline{\mathbb{Q}}_{\ell}\right) .
$$

The perversity of the right hand side then implies that $2 d-\left(2 \rho,\left|\mu_{\bullet}\right|\right) \leq-(2 \rho, \lambda)$. This implies that $d \leq\left(\rho,\left|\mu_{\bullet}\right|-\lambda\right)$. By induction on $\lambda$, we see

$$
\operatorname{dim} \operatorname{Gr}_{\leq \mu_{\bullet}}^{\lambda} \leq d+\operatorname{dim} \operatorname{Gr}_{\lambda}=\left(\rho,\left|\mu_{\bullet}\right|+\lambda\right)
$$

Remark 2.4. This argument also gives a canonical isomorphism

$$
V_{\mu_{\bullet}}^{\lambda}=\mathrm{H}_{c}^{\left(2 \rho,\left|\mu_{\bullet}\right|-\lambda\right)}\left(\operatorname{Gr}_{\mu_{\bullet}} \cap m^{-1}\left(\varpi^{\lambda}\right), \overline{\mathbb{Q}}_{\ell}\right) .
$$

Together with 4 , w.3. we see that there is a canonical basis of $V_{\mu_{\bullet}}^{\lambda}$ given by the set $\mathbb{B}_{\mu_{\bullet}}^{\lambda}$ of irreducible components of $\mathrm{Gr}_{\mu_{\bullet}} \cap m^{-1}\left(\varpi^{\lambda}\right)$ of dimension $\left(\rho,\left|\mu_{\bullet}\right|-\lambda\right)$.

By identifying $\left(\mathcal{A}_{1} \star \mathcal{A}_{2}\right) \star \mathcal{A}_{3}$ and $\mathcal{A}_{1} \star\left(\mathcal{A}_{2} \star \mathcal{A}_{3}\right)$ with $\mathcal{A}_{1} \star \mathcal{A}_{2} \star \mathcal{A}_{3}$, one equips $\mathrm{P}_{L^{+} G}(\mathrm{Gr})$ with a natural monoidal structure. The monoidal category $\left(\mathrm{P}_{L^{+} G}(\mathrm{Gr}), \star\right)$ is sometimes also denoted by $\mathrm{Sat}_{G}$ for simplicity.

\subsection{Semi-infinite orbits.}

2.2.1. Let $U \subset G$ be the unipotent radical of a chosen Borel subgroup $B$ of $G$. The affine Grassmannian $\mathrm{Gr}_{U}$ of $U$ is clearly represented by an inductive limit of perfect affine spaces. Recall the fact that $U \backslash G$ is quasi-affine, so Proposition 1.20 is applicable and $\operatorname{Gr}_{U} \subset \mathrm{Gr}_{G}$ is a locally closed embedding. Write $S_{0}=\operatorname{Gr}_{U} \subset \mathrm{Gr}_{G}$. For $\lambda \in \mathbb{X}_{\bullet}$, let $S_{\lambda}=L U \varpi^{\lambda}$ be the orbit through $\varpi^{\lambda}$. Then $S_{\lambda}=\varpi^{\lambda} \operatorname{Gr}_{U}$ and therefore is locally closed in $\operatorname{Gr}_{G}$. By the Iwasawa decomposition,

$$
\operatorname{Gr}_{G}=\bigcup_{\lambda \in \mathbb{X}} S_{\lambda}
$$

As in the equal characteristic situation,

Lemma 2.5. The closure $\bar{S}_{\lambda}=\cup_{\lambda^{\prime} \preceq \lambda} S_{\lambda^{\prime}}$, more precisely $\overline{S_{\lambda} \cap \mathrm{Gr}_{\leq \mu}}=\cup_{\lambda^{\prime} \preceq \lambda} S_{\lambda^{\prime}} \cap \mathrm{Gr}_{\leq \mu}$. 
Proof. The arguments of MV Proposition 3.1] do not apply directly because in mix characteristic one cannot attach to an affine root a map $\mathrm{SL}_{2} \rightarrow L G$. However, we can argue as follows. Let $\lambda$ be a coweight of $G$, and $\alpha$ be a simple coroot of $G$, with the corresponding simple root $\alpha^{\vee}$. It is enough to construct a $\mathbb{P}^{1, p^{-\infty}} \subset$ Gr such that $\infty \in \mathbb{P}^{1, p^{-\infty}}$ is $\varpi^{\lambda-\alpha}$ and the rest $\left(\mathbb{A}^{1}\right)^{p^{-\infty}} \subset S_{\lambda}$.

Let $i_{\alpha}: \mathrm{SL}_{2} \rightarrow G$ be the root $\mathrm{SL}_{2}$ of $G$ over $\mathcal{O}$ determined by $\alpha$. Choose a coweight $\lambda^{\prime}$ (of the adjoint group) such that $\left(\lambda^{\prime}, \alpha^{\vee}\right)=\left(\lambda, \alpha^{\vee}\right)-1=: m$. Then we obtain

$$
i_{\alpha, m}: L^{+} \mathrm{SL}_{2} \rightarrow L G, \quad g \mapsto \varpi^{\lambda^{\prime}} L^{+} i_{\alpha}(g) \varpi^{-\lambda^{\prime}}
$$

whose image is the subgroup generated by $\varpi^{\lambda^{\prime}} L^{+} U_{ \pm \alpha} \varpi^{-\lambda^{\prime}}$, where $U_{ \pm \alpha}$ are the root subgroups of $G$ over $\mathcal{O}$ corresponding to $\pm \alpha$. Let $Z=i_{\alpha, m}\left(L^{+} \mathrm{SL}_{2}\right) \varpi^{\lambda}$ be the $L^{+} \mathrm{SL}_{2}$-orbit through $\varpi^{\lambda}$. We claim that this is the desired $\mathbb{P}^{1, p^{-\infty}}$. Indeed, note that: (i) $\varpi^{\lambda^{\prime}} L^{+} U_{-\alpha} \varpi^{-\lambda^{\prime}}$ and $\varpi^{\lambda^{\prime}} L^{+} U_{\alpha}^{(1)} \varpi^{-\lambda^{\prime}}$ fix $\varpi^{\lambda}$, where we recall $U_{\alpha}^{(1)}$ denotes the first congruent group scheme of $U_{\alpha}$. Therefore, $i_{\alpha, m}\left(L^{+} \mathrm{SL}_{2}^{(1)}\right)$ acts trivially on $Z$; (ii) On the other hand, $\varpi^{\lambda^{\prime}} L^{+} U_{\alpha} \varpi^{-\lambda^{\prime}}$ do not fix $\varpi^{\lambda}$. From these two facts, we see that $Z \simeq \mathbb{P}^{1, p^{-\infty}}$ and $\left(\varpi^{\lambda^{\prime}} L^{+} U_{\alpha} \varpi^{-\lambda^{\prime}}\right) \varpi^{\lambda} \simeq$ $\left(\mathbb{A}^{1}\right)^{p^{-\infty}} \subset S_{\lambda}$. Finally, from the famous identity

$$
\left(\begin{array}{cc}
0 & -1 \\
1 & 0
\end{array}\right)=\left(\begin{array}{cc}
1 & -\varpi^{-1} \\
0 & 1
\end{array}\right)\left(\begin{array}{cc}
1 & 0 \\
\varpi & 1
\end{array}\right)\left(\begin{array}{cc}
1 & -\varpi^{-1} \\
0 & 1
\end{array}\right)\left(\begin{array}{cc}
\varpi^{-1} & 0 \\
0 & \varpi
\end{array}\right)
$$

we see that $\varpi^{\lambda^{\prime}} i_{\alpha}\left(\left(\begin{array}{cc}0 & -1 \\ 1 & 0\end{array}\right)\right) \varpi^{-\lambda^{\prime}} \varpi^{\lambda}=\left(\varpi^{\lambda-\alpha} \bmod L^{+} G\right)$, which is exactly the point $\infty \in \mathbb{P}^{1, p^{-\infty}}$.

Note that the restriction of the $L^{+} G$-torsor $L G \rightarrow \operatorname{Gr}_{G}$ over $S_{\lambda}$ has a canonical reduction as an $L^{+} U$-torsor given by $L U \rightarrow S_{\lambda}, n \mapsto \varpi^{\lambda} n \bmod L^{+} G$. Then it makes sense to talk about convolutions of these semi-infinite orbits. Let $\nu_{\bullet}$ be a sequence of (not necessarily dominant) coweights of $G$. One can define

$$
S_{\nu_{\bullet}}:=S_{\nu_{1}} \tilde{\times} S_{\nu_{2}} \tilde{\times} \cdots \tilde{\times} S_{\nu_{n}} \subset \operatorname{Gr} \tilde{\times} \operatorname{Gr} \tilde{\times} \cdots \tilde{\times} \mathrm{Gr} .
$$

The formula

$$
\left(\varpi^{\nu_{1}} x_{1}, \ldots, \varpi^{\nu_{n}} x_{n}\right) \mapsto\left(\varpi^{\nu_{1}} x_{1}, \varpi^{\nu_{1}+\nu_{2}}\left(\varpi^{-\nu_{2}} x_{1} \varpi^{\nu_{2}}\right) x_{2}, \ldots\right),
$$

defines an isomorphism

$$
m: S_{\nu_{\bullet}} \simeq S_{\nu_{1}} \times S_{\nu_{1}+\nu_{2}} \times \cdots \times S_{\left|\nu_{\bullet}\right|},
$$

as locally closed subset of $\operatorname{Gr} \tilde{\times} \mathrm{Gr} \tilde{x} \cdots \tilde{\times} \mathrm{Gr} \simeq \mathrm{Gr}^{n}$.

Note that each $S_{\nu_{i}} \cap \mathrm{Gr}_{\leq \mu_{i}}$ is $L^{+} U$-invariant, so it also makes sense to convolve the $L^{+} U$-spaces $S_{\nu_{i}} \cap \mathrm{Gr}_{\leq \mu_{i}}$, and there is the canonical isomorphism

$$
\left(S_{\nu_{1}} \cap \mathrm{Gr}_{\leq \mu_{1}}\right) \tilde{\times} \cdots \tilde{\times}\left(S_{\nu_{n}} \cap \mathrm{Gr}_{\leq \mu_{n}}\right) \simeq S_{\nu_{\bullet}} \cap \mathrm{Gr}_{\leq \mu_{\bullet}} .
$$

Remark 2.6. (i)Note that $S_{\nu} \cap \mathrm{Gr}_{\leq \mu}$ is closed in $S_{\nu}$ and therefore is a scheme. (ii)Unlike [NP, Lemma 9.1], the twisted product in l.h.s. of (2.2.3) does not split as product.

The Mirkovic-Vilonen theory exists in our situation. The key statement is the following

Proposition 2.7. For any $\mathcal{A} \in \mathrm{P}_{L^{+} G}\left(\operatorname{Gr}_{G}\right), \mathrm{H}_{c}^{i}\left(S_{\lambda}, \mathcal{A}\right)=0$ unless $i=(2 \rho, \lambda)$.

The proof of this proposition will be sketched in 2.2 .3 Note that the proof in $\mathrm{MV}$, Theorem 3.5] does not work in mixed characteristic.

Corollary 2.8. The perfect scheme $S_{\lambda} \cap \mathrm{Gr}_{\leq \mu}$ is equidimensional, of dimension $(\rho, \lambda+\mu)$. Its number of irreducible components equals to the dimension of the $\lambda$-weight space of the irreducible representation $V_{\mu}(\lambda)$ of $\hat{G}$ of highest weight $\mu$. 
Proof. First, we show that $S_{\lambda} \cap \mathrm{Gr}_{\leq \mu}$ is of dimension $(\rho, \lambda+\mu)$, and the number of irreducible components of maximal dimension equals to the the dimension of the $\lambda$-weight space of the irreducible representation $V_{\mu}(\lambda)$ of $\hat{G}$ of highest weight $\mu$. The proof is a special case of GHKR, Proposition 5.4.2]: it is enough to show that

$$
\lim _{q \rightarrow \infty} \frac{\left|\left(S_{\lambda} \cap \mathrm{Gr}_{\leq \mu}\right)\left(\mathbb{F}_{q}\right)\right|}{q^{(\rho, \lambda+\mu)}}=\operatorname{dim} V_{\mu}(\lambda) .
$$

One can replace $\mathrm{Gr}_{\leq \mu}$ in the above limit by the open cell $\mathrm{Gr}_{\mu}$. Now we regard $U(F)$ as a locally compact topological group and we normalize the measure on $U(F)$ so that the volume of $U(\mathcal{O})$ is one. Then one can express

$$
\left|\left(S_{\lambda} \cap \mathrm{Gr}_{\mu}\right)\left(\mathbb{F}_{q}\right)\right|=\int_{U(F)} 1_{G(\mathcal{O}) \varpi^{\mu} G(\mathcal{O})}\left(\varpi^{\lambda} u\right) d u .
$$

Recall the Satake isomorphism

$$
\text { Sat : } C_{c}^{\infty}(G(\mathcal{O}) \backslash G(F) / G(\mathcal{O})) \simeq C_{c}^{\infty}(T(F) / T(\mathcal{O}))^{W}=C\left[\mathbb{X}_{\bullet}(T)\right]^{W}
$$

is given by

$$
\operatorname{Sat}(f)\left(\varpi^{\lambda}\right)=q^{-(\rho, \lambda)} \int_{U(F)} f\left(\varpi^{\lambda} u\right) d u .
$$

Let $H_{\mu}$ denote the function on $C_{c}^{\infty}(G(\mathcal{O}) \backslash G(F) / G(\mathcal{O}))$ such that

$$
\operatorname{Sat}\left(H_{\mu}\right)\left(\varpi^{\lambda}\right)=\operatorname{dim} V_{\mu}(\lambda) \text {. }
$$

From the Lustzig-Kato polynomials,

$$
q^{-(\rho, \mu)} 1_{G(\mathcal{O}) \varpi^{\mu} G(\mathcal{O})}=H_{\mu}+\sum_{\nu<\mu} P_{\mu \nu}\left(q^{-1}\right) H_{\nu},
$$

where $P_{\mu \lambda}(v)$ is some polynomial of $v$ without the constant coefficient. Combining (2.2.5), (2.2.6) and (2.2.7)

$$
\frac{\left|\left(S_{\lambda} \cap \mathrm{Gr}_{\mu}\right)\left(\mathbb{F}_{q}\right)\right|}{q^{(\rho, \lambda+\mu)}}=\operatorname{dim} V_{\mu}(\lambda)+\sum_{\nu<\mu} c_{\mu \nu}\left(q^{-1}\right) \operatorname{dim} V_{\nu}(\lambda) .
$$

As $q \rightarrow \infty$, the error term goes to zero, and the main term becomes $\operatorname{dim} V^{\mu}(\lambda)$.

Then one can also follow GHKR, Lemma 2.17.4] to deduce the equidimensionality of $S_{\lambda} \cap \mathrm{Gr}_{\leq \mu}$ from the the upper bounds of the dimension of $S_{\lambda} \cap \mathrm{Gr}_{\leq \mu}$ and Proposition 2.7 .

Let $\mathbb{B}_{\mu}(\lambda)$ denote the set of irreducible components of $S_{\lambda} \cap \mathrm{Gr}_{\leq \mu}$. More generally, let $\mathbb{B}_{\mu_{\bullet}}(\lambda)$ denote the set of irreducible components of $m^{-1}\left(S_{\lambda}\right) \cap \mathrm{Gr}_{\leq \mu_{\bullet}}$. Another two corollaries of Theorem 2.7 are

Corollary 2.9. There is a canonical isomorphism

$$
\mathrm{H}_{c}^{i}\left(S_{\lambda}, \mathrm{IC}_{\mu}\right)= \begin{cases}\overline{\mathbb{Q}}_{\ell}\left[\mathbb{B}_{\mu}(\lambda)\right] & i=2(\rho, \lambda) \\ 0 & \text { otherwise. }\end{cases}
$$

More precisely, the cycle classes of irreducible components of $S_{\lambda} \cap \mathrm{Gr}_{\leq \mu}$ form a basis of $\mathrm{H}_{c}^{i}\left(S_{\lambda}, \mathrm{IC}_{\mu}\right)$.

Proof. Same argument as [MV, Proposition 3.10].

Corollary 2.10. There is a canonical isomorphism

$$
\mathrm{H}^{*}\left(\mathrm{Gr}_{G},-\right) \simeq \bigoplus_{\lambda} \mathrm{H}_{c}^{*}\left(S_{\lambda},-\right): \mathrm{P}_{L^{+} G}\left(\operatorname{Gr}_{G}\right) \rightarrow \operatorname{Vect}_{\overline{\mathbb{Q}}_{\ell}} .
$$

In addition, $\mathrm{H}^{*}\left(\mathrm{Gr}_{G},-\right)$ is faithful.

Proof. Same argument as [MV, Theorem 3.6, Corollary 3.7]. 
2.2.2. We discuss the geometry of $\mathrm{Gr}_{<\mu}$ for $\mu$ minuscule or quasi-minuscule, similar to $\mathrm{NP}$, $\S 6-\S 8]$, but with a few justifications. Denote by $\bar{G}=G \otimes_{\mathcal{O}} k$ the special fiber of $G$, which is a reductive group over $k$, with $\bar{U} \subset \bar{B} \subset \bar{G}$. A fundamental coweight $\mu$ of $G$ is called minuscule (resp. quasi-minuscule) if $\langle\alpha, \mu\rangle \leq 1$ (resp. $\leq 2$ ) for every positive root $\alpha$ of $G$. If $G$ is a simple group and is not of type $A$, then the quasi-minuscule coweight is the unique short dominant coroot. We will first show that

Lemma 2.11. Proposition 2.7 hold for minuscule or quasi-minuscule $\mu$.

In fact, we will show directly Corollary 2.9 holds in these cases.

If $\mu$ is minuscule coweight of $G, \mathrm{Gr}_{\leq \mu}=\mathrm{Gr}_{\mu}=\left(\bar{G} / \bar{P}_{-\mu}\right)^{p^{-\infty}}$, where $\bar{P}_{-\mu}$ is the parabolic subgroup of $\bar{G}$ whose roots are those $\alpha$ with $\langle\alpha, \mu\rangle \leq[11$. In this case

$$
S_{\lambda} \cap \mathrm{Gr}_{\mu}= \begin{cases}\emptyset & \lambda \notin W \mu \\ \left(\bar{U} w \bar{P}_{-\mu} / \bar{P}_{-\mu}\right)^{p^{-\infty}} & \lambda=w \mu\end{cases}
$$

is irreducible, isomorphic to the perfection of an affine space. Then Lemma 2.11 is clear in this case.

Next we assume that $\mu=\theta$ is quasi minuscule. Several discussions in NP need justifications in this case. We denote the corresponding root by $\theta^{\vee}$, which is the highest root of $G$. We first construct "resolution" of $\mathrm{Gr}_{\leq \mu}$. The one given in loc. cit. does not work in mixed characteristic. Our construction is different, and arises as a discussion with X. He.

We fix the apartment $A(G, F)$ corresponding to the torus $T$, and identify $A(G, F) \simeq$ $\mathbb{X} .\left(T_{\text {ad }}\right) \otimes \mathbb{R}$ via the hyperspacial point $v_{0} \in A(G, F)$ corresponding to $G$ over $\mathcal{O}$. For $r \in[0,1]$ be a real number, we denote the (perfection of the) parahoric of $G$ given by the point $-r \mu$ by $Q_{r}$. Note that

(1) $Q_{0}=L^{+} G$ and $Q_{1}=\varpi^{\mu} L^{+} G \varpi^{-\mu}$.

(2) $Q_{\frac{1}{2}}$ is a maximal parahoric. Namely, the point $-\mu / 2$ is a vertex of $A(G, F)$, which is the intersection of the interval $[0,-\mu]$ and the hyperplane $H$ given by the affine root $1+\theta^{\vee}$.

(3) $Q_{\frac{1}{4}} \subset Q_{0} \cap Q_{\frac{1}{2}}$ and $Q_{\frac{3}{4}} \subset Q_{\frac{1}{2}} \cap Q_{1}$.

Lemma 2.12. (i) The quotient $Q_{\frac{1}{2}} / Q_{\frac{3}{4}}$ is isomorphic to $\mathbb{P}^{1, p^{-\infty}}$.

(ii) The map

$$
\pi_{\mu}: \widetilde{\mathrm{Gr}}_{\leq \mu}:=Q_{0} \times{ }^{Q_{\frac{1}{4}}} Q_{\frac{1}{2}} / Q_{\frac{3}{4}} \rightarrow \mathrm{Gr}_{\leq \mu}, \quad\left(g, g^{\prime}\right) \mapsto g g^{\prime} \varpi^{\mu}
$$

restricts to an isomorphism

$$
\stackrel{\pi}{\pi}: Q_{0} \times{ }^{Q_{\frac{1}{4}}}\left(Q_{\frac{1}{4}} Q_{\frac{3}{4}}\right) / Q_{\frac{3}{4}} \simeq \mathrm{Gr}_{\mu},
$$

and contracts

$$
\pi_{0}:\left(\bar{G} / \bar{P}_{-\mu}\right)^{p^{-\infty}} \simeq Q_{0} \times{ }^{Q_{\frac{1}{4}}} Q_{\frac{1}{4}} s_{1+\theta} Q_{\frac{3}{4}} / Q_{\frac{3}{4}} \rightarrow \mathrm{Gr}_{0}=\{1\},
$$

where $s_{1+\theta}$ is the affine reflection corresponding to $1+\theta$.

Proof. For (i), it is enough to observe that the only affine root appearing in $Q_{\frac{1}{2}}$ but not in $Q_{\frac{1}{4}}$ is $-\theta-1$. For (ii), note that $Q_{0} \cap Q_{\frac{3}{4}}=Q_{0} \cap Q_{1}$. Therefore, the statement for $\pi$ holds. The statement for $\pi_{0}$ is clear.

Let us write

$$
\phi: \widetilde{\mathrm{Gr}}_{\leq \mu} \rightarrow Q_{0} / Q_{\frac{1}{4}}=\left(\bar{G} / \bar{P}_{-\mu}\right)^{p^{-\infty}}, \quad \stackrel{\circ}{\phi}: \mathrm{Gr}_{\mu} \stackrel{\stackrel{\circ}{\pi}^{-1}}{\rightarrow} Q_{0} \times{ }^{Q_{\frac{1}{4}}}\left(Q_{\frac{1}{4}} Q_{\frac{3}{4}}\right) / Q_{\frac{3}{4}} \rightarrow Q_{0} / Q_{\frac{1}{4}}
$$

\footnotetext{
${ }^{11} \mathrm{In}\left[\mathrm{NP}, \bar{P}_{-\mu}\right.$ is denoted by $P_{\mu}$.
} 
XINWEN ZHU

where $\bar{P}_{-\mu}$ as before is the parabolic of $\bar{G}$ whose roots are those $\alpha$ with $\langle\alpha, \mu\rangle \leq 0$. Let $\Delta_{\theta}$ denote the subset of simple coroots that are conjugate to $\theta$ under the action of the Weyl group. If $G$ is a simple group, then $\Delta_{\theta}$ is the set of short simple coroots.

Now we study $S_{\lambda} \cap \mathrm{Gr}_{\leq \mu}$. If $\lambda=w \mu$ for some $w \in W$, it is easy to see that $S_{\lambda} \cap \mathrm{Gr}_{\leq \mu}=$ $U(\mathcal{O}) \varpi^{\lambda}$, from which one deduces: if $\lambda=w \mu$ is a positive coroot, then

$$
S_{\lambda} \cap \mathrm{Gr}_{\leq \mu}=S_{\lambda} \cap \mathrm{Gr}_{\mu}=\dot{\phi}^{-1}\left(\bar{U} w \bar{P}_{-\mu} / \bar{P}_{-\mu}\right)^{p^{-\infty}} .
$$

If $\lambda=w \mu$ is a negative coroot, then still $S_{\lambda} \cap \mathrm{Gr}_{\leq \mu}=S_{\lambda} \cap \mathrm{Gr}_{\mu}$ and

$$
\dot{\phi}: S_{\lambda} \cap \mathrm{Gr}_{\leq \mu} \simeq\left(\bar{U} w \bar{P}_{-\mu} / \bar{P}_{-\mu}\right)^{p^{-\infty}} .
$$

Finally,

$$
S_{0} \cap \mathrm{Gr}_{\leq \mu}=\pi\left(\phi^{-1}\left(\bigcup_{w \mu<0} \bar{U} w \bar{P}_{-\mu} / \bar{P}_{-\mu}\right)^{p^{-\infty}}\right) \backslash \bigcup_{w \mu<0}\left(S_{w \mu} \cap \mathrm{Gr}_{\leq \mu}\right) .
$$

There is a canonical bijection between $\Delta_{\theta}$ and the set of irreducible components of $S_{0} \cap \mathrm{Gr}_{\leq \mu}$ given as follows: $\alpha \in \Delta_{\theta}$ correspondes to the unique irreducible component of $S_{0} \cap \mathrm{Gr}_{\leq \mu}$ given by

$$
\left(S_{0} \cap \mathrm{Gr}_{\leq \mu}\right)^{\alpha}:=\operatorname{Gr}_{0} \bigcup \pi\left(\phi^{-1}\left(\bar{U} w \bar{P}_{-\mu} / \bar{P}_{-\mu}\right)^{p^{-\infty}}\right) \backslash\left(S_{w \mu} \cap \mathrm{Gr}_{\leq \mu}\right),
$$

where $w \mu=-\alpha$. Now we show that Proposition 2.11 also holds in this situation. This is clear for $\lambda=w \mu$. It remains to consider the case $S_{0} \cap \mathrm{Gr}_{\leq \mu}$. Let $d=(2 \rho, \mu)$. We will ignore the Tate twist in the sequel.

According to the decomposition theorem (applying to certain model of $\pi: \widetilde{\mathrm{Gr}}_{\leq \mu} \rightarrow \mathrm{Gr}_{\leq \mu}$ ), we have

$$
\pi_{*} \overline{\mathbb{Q}}_{\ell}[d]=\mathrm{IC}_{\mu} \oplus \mathcal{C}
$$

where $\mathcal{C}$ is certain complex of vector spaces supported at $\operatorname{Gr}_{0}$. One has

$$
\mathrm{H}^{i}(\mathcal{C})= \begin{cases}\mathrm{H}^{i+d-2}\left(\bar{G} / \bar{P}_{-\mu}\right) & i<0 \\ \mathrm{H}^{i+d}\left(\bar{G} / \bar{P}_{-\mu}\right) & i \geq 0 .\end{cases}
$$

Indeed, the second equality follows from the fact the stalk cohomology of $\mathrm{IC}_{\mu}$ is concentrated on the negative degrees, and the first equality follows from the second by duality.

On the other hand, we have

$$
R \Gamma_{c}\left(\pi^{-1}\left(S_{0} \cap \mathrm{Gr}_{\leq \mu}\right), \overline{\mathbb{Q}}_{\ell}[d]\right)=R \Gamma_{c}\left(S_{0}, \mathrm{IC}_{\mu}\right) \oplus \mathcal{C} .
$$

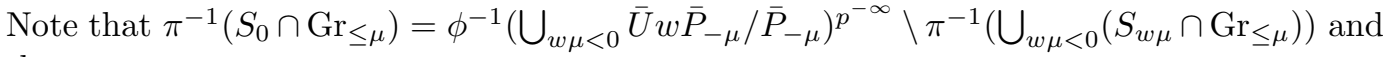
the map

$$
\phi: \phi^{-1}\left(\bigcup_{w \mu<0} \bar{U} w \bar{P}_{-\mu} / \bar{P}_{-\mu}\right)^{p^{-\infty}} \rightarrow \bigcup_{w \mu<0} \bar{U} w \bar{P}_{-\mu} / \bar{P}_{-\mu}
$$

is a $\mathbb{P}^{1}$-fibration (up to perfection). In addition, $\pi^{-1}\left(\bigcup_{w \mu<0}\left(S_{w \mu} \cap \mathrm{Gr}_{\leq \mu}\right)\right.$ can be regarded as a section of this map.

Therefore,

$$
\left.R \Gamma_{c}\left(\pi^{-1}\left(S_{0} \cap \mathrm{Gr}_{\leq \mu}\right), \overline{\mathbb{Q}}_{\ell}[d]\right)=R \Gamma_{c}\left(\bigcup_{w \mu<0} \bar{U} w \bar{P}_{-\mu} / \bar{P}_{-\mu}, \overline{\mathbb{Q}}_{\ell}[d-2]\right)\right) .
$$

To prove Proposition 2.11 for $S_{0} \cap \mathrm{Gr}_{\leq \mu}$, it remains to compare (2.2.9) and (2.2.10). However, note that the right hand sides of both equalities only involve the group $\bar{G}$, defined over the finite field $\mathbb{F}_{q}$. Therefore, one can apply the computation in $[\mathrm{NP}, \S 8]$ directly to see that $\mathrm{H}^{i}(\mathcal{C})=\mathrm{H}_{c}^{i}\left(\pi^{-1}\left(S_{0} \cap \mathrm{Gr}_{\leq \mu}\right)\right)$ for $i \neq 0$ and if $i=0$,

$$
\mathrm{H}^{0}\left(S_{0}, \mathrm{IC}_{\mu}\right) \simeq \overline{\mathbb{Q}}^{\left|\Delta_{\theta}\right|}
$$

This finishes the proof of Proposition 2.11. 
2.2.3. Now combining the proof of Proposition 2.11 with (2.2.3), we have the following corollaries, whose proofs are as in [NP, 9.2-9.4]. Let $M$ be the set of minimal elements in $\mathbb{X}_{\bullet}^{+} \backslash\{0\}$. It is the union of minuscule coweights and the quasi-minscule coweights.

Corollary 2.13. Let $\mu_{\bullet}=\left(\mu_{1}, \ldots, \mu_{m}\right) \subset M$. Then for any $\lambda_{\bullet}, S_{\lambda_{\bullet}} \cap \mathrm{Gr}_{\leq \mu}$ is equidimensional, and

$$
\operatorname{dim}\left(S_{\lambda_{\bullet}} \cap \operatorname{Gr}_{\leq \mu_{\bullet}}\right)=\left(\rho,\left|\lambda_{\bullet}\right|+\left|\mu_{\bullet}\right|\right) .
$$

Corollary 2.14. Let $\mu_{\bullet} \subset M$. Then the map $\pi: \mathrm{Gr}_{\leq \mu_{\bullet}} \rightarrow \mathrm{Gr}_{\leq\left|\mu_{\bullet}\right|}$ is semi-small. So $\mathrm{IC}_{\mu_{1}} \star \cdots \star \mathrm{IC}_{\mu_{m}}$ is perverse.

In addition, we have the following corollary. The argument is similar to the proof of [NP, Theorem 3.1] given at the beginning of $\S 11$ of loc. cit.. But one justification is needed.

Corollary 2.15. For any $\lambda, R \Gamma_{c}\left(S_{\lambda}, \mathrm{IC}_{\mu_{1}} \star \cdots \star \mathrm{IC}_{\mu_{m}}\right)$ is concentrated on degree $(2 \rho, \lambda)$.

Proof. Note that $\pi^{-1} S_{\lambda}=\bigsqcup_{\nu_{\bullet}\left|\nu_{\boldsymbol{\bullet}}\right|=\lambda} S_{\nu_{\bullet}}$. It is enough to show that $R \Gamma_{c}\left(S_{\nu_{\bullet}}, \mathrm{IC}_{\mu_{1}} \star \cdots \star\right.$ $\left.\mathrm{IC}_{\mu_{m}}\right)$ is concentrated on degree $(2 \rho, \lambda)$. But by (2.2.3), together with Proposition 2.11, it remains to show

$$
\begin{aligned}
R \Gamma_{c}\left(\left(S_{\lambda_{1}} \cap \mathrm{Gr}_{\leq \mu_{1}}\right) \tilde{\times} \cdots \tilde{\times}\left(S_{\lambda_{n}} \cap \mathrm{Gr}_{\leq \mu_{n}}\right), \mathrm{IC}_{\mu_{1}} \tilde{\otimes} \cdots \tilde{\otimes} \mathrm{IC}_{\mu_{m}}\right) & \\
& =R \Gamma_{c}\left(S_{\lambda_{1}}, \mathrm{IC}_{\mu_{1}}\right) \otimes \cdots \otimes R \Gamma_{c}\left(S_{\lambda_{m}}, \mathrm{IC}_{\mu_{m}}\right) .
\end{aligned}
$$

Although the twisted product $\left(S_{\lambda_{1}} \cap \mathrm{Gr}_{\leq \mu_{1}}\right) \tilde{\times} \cdots \tilde{\times}\left(S_{\lambda_{n}} \cap \mathrm{Gr}_{\leq \mu_{n}}\right)$ may not split as a product, but as $L^{+} U$ is the (perfection) of a connected pro-algebraic group, the cohomology splits.

Corollary 2.14 allows us to define a full additive category of $\mathrm{Sat}_{G}$, spanned by objects isomorphic to $\mathrm{IC}_{\mu_{1}} \star \cdots \star \mathrm{IC}_{\mu_{m}}$ for $\mu_{\bullet} \in M$. Let us denote this subcategory by Sat ${ }_{G}^{0}$. Note that $\mathrm{Sat}_{G}^{0}$ is in fact a monoidal subcategory of $\operatorname{Sat}_{G}$ under the convolution. We have

Lemma 2.16. As a monoidal abelian category, $\mathrm{Sat}_{G}$ is the idempotent completion of $\mathrm{Sat}_{G}^{0}$. Concretely, every $\mathrm{IC}_{\mu}$ appears as a direct summand of $\mathrm{IC}_{\mu_{1}} \star \cdots \star \mathrm{IC}_{\mu_{m}}$ for $\mu_{\bullet} \in M$.

This is a geometric version of the so-called PRV conjecture. The argument as in $\mathbb{N P}$, Proposition 9.6] applies here.

Note that this proposition together with Corollary 2.15 implies Proposition 2.7, and together with Corollary 2.14 implies Proposition 2.2.

\subsection{Monoidal structure on $\mathrm{H}^{*}$.}

2.3.1. We endow the global cohomology functor

$$
\mathrm{H}^{*}(-):=\mathrm{H}^{*}(\mathrm{Gr},-): \operatorname{Sat}_{G} \rightarrow \operatorname{Vect}_{\overline{\mathbb{Q}}_{\ell}}
$$

with a monoidal structure. In the equal characteristic situation, such a monoidal structure is provided by identifying convolution products with fusion products coming from a global curve (cf. $\mathrm{MV}$ and $[\mathrm{BD}, \S 5.3]$ ). If in addition, $k=\mathbb{C}$, one can endow $\mathrm{H}^{*}$ with another monoidal structure by identifying $\mathrm{Gr}_{G}^{b}$ with the based loop space of a maximal compact subgroup of $G$ and identifying the convolution product of the affine Grassmannian with the multiplication of the loop group (cf. Gi]). Neither method applies directly in our setting so we need a third construction. It is not hard to check that in the equal characteristic situation, all three monoidal structures coincide.

Recall that for $\mathcal{A} \in \mathrm{Sat}_{G}$, it makes sense to consider its $L^{+} G$-equivariant cohomology $\mathrm{H}_{L^{+} G}^{*}(\mathcal{A})$, which is an $R_{\bar{G}, \ell^{-}}$module (see A.3.5). But as is well-known, there is another

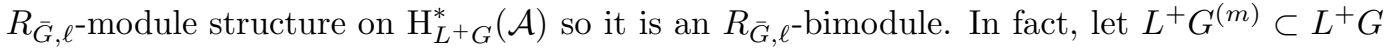
denote the $m$ th congruence subgroup, and let $\mathrm{Gr}^{(m)}=L G / L^{+} G^{(m)}$ denote the universal 
$L^{m} G$-torsor on Gr. Then $\mathrm{Gr}^{(m)}$ admits an action of $L^{+} G \times L^{m} G$ and the projection $\pi_{m}$ : $\mathrm{Gr}^{(m)} \rightarrow \mathrm{Gr}$ is $L^{+} G$-equivariant. Then by (A.3.5),

$$
\mathrm{H}_{L^{+}{ }_{G}}^{*}(\mathcal{A}) \simeq \mathrm{H}_{L^{+}{ }_{G \times L^{m} G}}^{*}\left(\pi_{m}^{*} \mathcal{A}\right)
$$

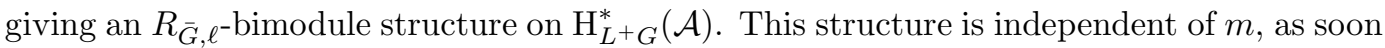
as $m>0$. The category of $R_{\bar{G}, \ell}$-bimodules have a natural monoidal structure.

Lemma 2.17. There is a natural monoidal structure on $\mathrm{H}_{L+G}^{*}(-): \operatorname{Sat}_{G} \rightarrow\left(\mathrm{R}_{\bar{G}, \ell} \otimes \mathrm{R}_{\bar{G}, \ell}\right)$-mod. I.e., for every $\mathcal{A}_{1}, \mathcal{A}_{2}, \ldots, \mathcal{A}_{n}$, there is a canonical isomorphism of $R_{\bar{G}, \ell^{-} \text {bimodules }}$

$$
\mathrm{H}_{L^{+} G}^{*}\left(\mathcal{A}_{1} \star \cdots \star \mathcal{A}_{n}\right) \simeq \mathrm{H}_{L^{+} G^{*}}^{*}\left(\mathcal{A}_{1}\right) \otimes_{R_{\bar{G}, \ell}} \cdots \otimes_{R_{\bar{G}, \ell}} \mathrm{H}_{L^{+} G}^{*}\left(\mathcal{A}_{n}\right),
$$

satisfying the natural compatibility conditions.

Proof. This is standard (in the spirit of Soergel bimodules) and we sketch a proof. For $Z \subset$ Gr a closed subset, let $Z^{(m)}$ denote its premage in $\mathrm{Gr}^{(m)}$. We choose $m_{1}, \ldots, m_{n}$, such that $m_{n}=1$ and $L^{+} G$ acts on $\operatorname{Supp}\left(\mathcal{A}_{i}\right)^{\left(m_{i}\right)}$ via $L^{+} G \rightarrow L^{m_{i-1}} G$. Then there is an $L^{+} G \times \prod_{i} L^{m_{i}} G$-equivariant projection

$$
\prod_{i} \operatorname{Supp}\left(\mathcal{A}_{i}\right)^{\left(m_{i}\right)} \rightarrow \operatorname{Supp}\left(\mathcal{A}_{1}\right) \tilde{\times} \cdots \tilde{\times} \operatorname{Supp}\left(\mathcal{A}_{n}\right),
$$

where $L^{+} G$ acts by left multiplication, $L^{m_{i}} G$ diagonally acts on $\operatorname{Supp}\left(\mathcal{A}_{i}\right)^{\left(m_{i}\right)} \times \operatorname{Supp}\left(\mathcal{A}_{i+1}\right)^{\left(m_{i+1}\right)}$ from the middle. This induces a canonical isomorphism

$$
\mathrm{H}_{L^{+} G}^{*}\left(\mathcal{A}_{1} \star \cdots \star \mathcal{A}_{n}\right) \simeq \mathrm{H}_{L^{+} G \times \prod L^{m_{i}}{ }^{*}}\left(\bigotimes_{i} \pi_{m_{i}}^{*} \mathcal{A}_{i}\right) .
$$

On the other hand, the $L^{+} G \times \prod_{i} L^{m_{i}} G$-equivariant projection

$$
\prod_{i} \operatorname{Supp}\left(\mathcal{A}_{i}\right)^{\left(m_{i}\right)} \rightarrow \prod_{i} \operatorname{Supp}\left(\mathcal{A}_{i}\right),
$$

where $L^{+} G$ acts on $\operatorname{Supp}\left(\mathcal{A}_{1}\right)$, and $L^{m_{i}} G$ acts on $\operatorname{Supp}\left(\mathcal{A}_{i+1}\right)$ by left multiplication, induces a map

$$
\mathrm{H}_{L+G}^{*}\left(\mathcal{A}_{1}\right) \otimes_{R_{\bar{G}, \ell}} \cdots \otimes_{R_{\bar{G}, \ell}} \mathrm{H}_{L^{+} G}^{*}\left(\mathcal{A}_{n}\right) \rightarrow \mathrm{H}_{L+G \times \prod L^{m_{i}} G^{*}}\left(\bigotimes_{i} \pi_{m_{i}}^{*} \mathcal{A}_{i}\right) .
$$

The composition of (2.3.1) and (2.3.2) gives

$$
\mathrm{H}_{L^{+} G}^{*}\left(\mathcal{A}_{1}\right) \otimes_{R_{\bar{G}, \ell}} \cdots \otimes_{R_{\bar{G}, \ell}} \mathrm{H}_{L^{+} G}^{*}\left(\mathcal{A}_{n}\right) \rightarrow \mathrm{H}_{L^{+} G}^{*}\left(\mathcal{A}_{1} \star \cdots \star \mathcal{A}_{n}\right),
$$

which is an isomorphism by an easy spectral sequence argument. Its inverse then gives the desired isomorphism, which is clearly compatible with the associativity constraints.

2.3.2. To continue, we make the following observation. We use the notation as in $\$ 1.5 .2$ Let $A(G, F)$ be the apartment of the building of $G$ corresponding to the torus $T$. Let $\widetilde{W}$ denote the Iwahori-Weyl group. Let $\bar{W}=\widetilde{W} / \mathbb{X}$ • denote the finite Weyl group, i.e. the Weyl group of $G$. For a point $x \in A(G, F)$, let $\bar{L}_{x}$ denote the reductive quotient of the parahoric group scheme determined by $x$. Then $\bar{T} \subset \bar{L}_{x}$. Let $W_{x} \subset \widetilde{W}$ denote the Weyl group of $\bar{L}_{x}$, and $\bar{W}_{x}$ be its image in $\bar{W}$. Then

$$
R_{\bar{L}_{x}, \ell}=R_{\bar{T}_{x}, \ell}^{\bar{W}_{x}}
$$

In particular, if $v$ is a hyperspecial vertex, $R_{\bar{L}_{v}, \ell}=R_{\bar{T}, \ell}^{\bar{W}}=R_{\bar{G}, \ell}$ is a subring of $R_{\bar{L}_{x}, \ell}$ for any $x \in A(G, F)$. On the other hand, all $R_{\bar{L}_{x}, \ell}$ are contained in $R_{\bar{T}, \ell}$.

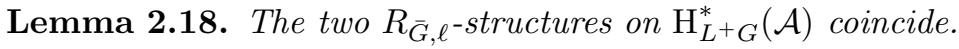


Proof. According to Lemma 2.16 and Lemma 2.17 it is enough to prove this for $\mathcal{A}=\mathrm{IC}_{\mu}$, and $\mu$ is minuscule or quasi-minuscule. We first consider the case when $\mu$ is quasi-minuscule. Let $\widetilde{\mathrm{Gr}}_{\leq \mu}$ be as in Lemma 2.12. Following the notation there (in particular identifying $A(G, F)$ with $\mathbb{X}_{\bullet}\left(T_{\text {ad }}\right)_{\mathbb{R}}$ using the hyperspecial vertex $v_{0}$ corresponding to $\left.G / \mathcal{O}\right)$,

$$
\widetilde{\mathrm{Gr}}_{\leq \mu}=Q_{0} \times{ }^{Q_{1 / 4}} Q_{1 / 2} \times{ }^{Q_{3 / 4}} Q_{1} / Q_{1} .
$$

and therefore by the same argument as in Lemma 2.17

$$
\mathrm{H}_{L+G}^{*}\left(\widetilde{\mathrm{Gr}}_{\leq \mu}\right)=R_{\bar{L}_{-\mu / 4}, \ell} \otimes_{R_{\bar{L}_{-\mu / 2}, \ell}} R_{\bar{L}_{-3 \mu / 4}, \ell},
$$

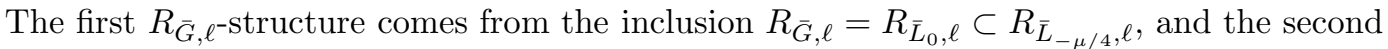
comes from the map $R_{\bar{G}, \ell}=R_{\bar{L}_{-\mu}, \ell} \subset R_{\bar{L}_{-3 \mu / 4}, \ell}$. But as $R_{\bar{G}, \ell}$ is a subring of $R_{\bar{L}_{-\mu / 2}, \ell}$, these two $R_{\bar{G}, \ell}$ structures coincide. It implies the two $R_{\bar{G}, \ell^{-}}$structures on $\mathrm{IH}_{L^{+} G}\left(\mathrm{Gr}_{\leq \mu}\right)=$ $\mathrm{H}_{L^{+}{ }_{G}}^{*}\left(\mathrm{IC}_{\mu}[-(2 \rho, \mu)]\right)$ coincide, as it is direct summand of $\mathrm{H}_{L^{+}{ }_{G}}^{*}\left(\widetilde{\mathrm{Gr}}_{\leq \mu}\right)$.

If $\mu$ is minuscule, one can argue similarly,

$$
\mathrm{H}_{L^{+} G}^{*}\left(\mathrm{Gr}_{\mu}\right)=R_{\bar{L}_{-\mu / 2}, \ell}
$$

with the two $R_{\bar{G}, \ell}$ structures given by $R_{\bar{G}, \ell}=R_{\bar{L}_{0}, \ell} \subset R_{\bar{L}_{-\mu / 2}, \ell}$ and $R_{\bar{G}, \ell}=R_{\bar{L}_{-\mu, \ell}} \subset$ $R_{\bar{L}_{-\mu / 2, \ell}}$, which clearly coincide.

Note that there is a canonical isomorphism $\mathrm{H}^{*}(\mathcal{A})=\overline{\mathbb{Q}}_{\ell} \otimes_{R_{\bar{G}, \ell}} \mathrm{H}_{L^{+} G}(\mathcal{A})$, where $R_{\bar{G}, \ell} \rightarrow \overline{\mathbb{Q}}_{\ell}$ is via the augmentation map, again by an easy spectral sequence argument. Combining the above two lemmas, we have

Proposition 2.19. The hypercohomology functor $\mathrm{H}^{*}(-):=\mathrm{H}^{*}\left(\mathrm{Gr}_{G},-\right): \operatorname{Sat}_{G} \rightarrow \operatorname{Vect}_{\overline{\mathbb{Q}}_{\ell}}$ is a natural monoidal functor.

\subsection{The commutativity constraints.}

2.4.1. In this subsection, we endow $\mathrm{Sat}_{G}$ with the commutativity constraints. The main statement is

Proposition 2.20. For every $\mathcal{A}_{1}, \mathcal{A}_{2} \in \mathrm{Sat}_{G}$, there exists a unique isomorphism $c_{\mathcal{A}_{1}, \mathcal{A}_{2}}$ : $\mathcal{A}_{1} \star \mathcal{A}_{2} \simeq \mathcal{A}_{2} \star \mathcal{A}_{1}$ such that the following diagram is commutative

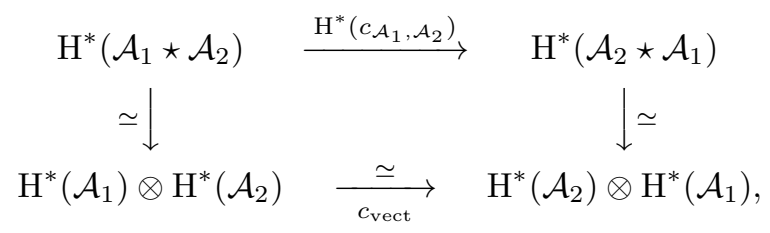

where the vertical isomorphisms come from Proposition 2.19, and the isomorphism $c_{\mathrm{vect}}$ in the bottom row is the usual commutativity for vector spaces.

As $\mathrm{H}^{*}: \mathrm{Sat}_{G} \rightarrow \operatorname{Vect}_{\overline{\mathbb{Q}}_{\ell}}$ is faithful, the uniqueness of $c_{\mathcal{A}_{1}, \mathcal{A}_{2}}$ is clear. The content is its existence. This proposition will be proved in the rest of the subsection. We first give its consequence.

Corollary 2.21. The monoidal category $\mathrm{Sat}_{G}$, equipped with the above constraints $c_{\mathcal{A}_{1}, \mathcal{A}_{2}}$, form a symmetric monoidal category. The hypercohomology functor $\mathrm{H}^{*}$ is a tensor functor.

Proof. The proof of the first statement follows the idea of Ginzburg (cf. Gil). Namely, we need to check $c_{\mathcal{A}_{2}, \mathcal{A}_{1}} c_{\mathcal{A}_{1}, \mathcal{A}_{2}}=\mathrm{id}$, and the hexagon axioms. Using the faithfulness of $\mathrm{H}^{*}$, it is enough to prove these after taking the cohomology. Using Proposition 2.20, and the fact $c_{\text {vect }}^{2}=\mathrm{id}$, we see that $\mathrm{H}^{*}\left(c_{\mathcal{A}_{2}, \mathcal{A}_{1}} c_{\mathcal{A}_{1}, \mathcal{A}_{2}}\right)=\mathrm{id}$, and therefore $c_{\mathcal{A}_{2}, \mathcal{A}_{1}} c_{\mathcal{A}_{1}, \mathcal{A}_{2}}=\mathrm{id}$. The hexagon axioms can be proved similarly. The second statement is clear. 
2.4.2. In order to construct $c_{\mathcal{A}_{1}, \mathcal{A}_{2}}$, we need some preparations.

Let

$$
\operatorname{Gr}_{G}^{\mathrm{op}}:=L^{+} G \backslash L G
$$

on which $L^{+} G$ acts by right multiplication. Let $\mathrm{P}_{L^{+} G}\left(\mathrm{Gr}_{G}^{\mathrm{op}}\right)$ denote the corresponding category of equivariant perverse sheaves. Note that $\mathrm{P}_{L+G}\left(\mathrm{Gr}_{G}^{\mathrm{op}}\right)$ also has a monoidal structure: There is the convolution Grassmannian

$$
\mathrm{Gr}^{\mathrm{op}} \tilde{\times} \mathrm{Gr}^{\mathrm{op}}:=L^{+} G \backslash L G \times{ }^{L^{+} G} L G
$$

equipped with $\left(m, \mathrm{pr}_{2}\right): \mathrm{Gr}^{\text {op }} \tilde{\times} \mathrm{Gr}^{\text {op }} \rightarrow \mathrm{Gr}^{\text {op }} \times \mathrm{Gr}^{\text {op }}$. Then for $\mathcal{A}_{1}, \mathcal{A}_{2} \in \mathrm{P}_{L^{+} G}\left(\mathrm{Gr}_{G}^{\text {op }}\right)$, one forms the twisted product $\mathcal{A}_{1} \tilde{\otimes} \mathcal{A}_{2}$ whose pullback along $\mathrm{Gr}^{\mathrm{op}} \times L G \rightarrow \mathrm{Gr}^{\mathrm{op}} \tilde{\times} \mathrm{Gr}^{\mathrm{op}}$ is the pullback of $\mathcal{A}_{1} \otimes \mathcal{A}_{2}$ along $\mathrm{Gr}^{\mathrm{op}} \times L G \rightarrow \mathrm{Gr}^{\mathrm{op}} \times \mathrm{Gr}^{\mathrm{op}}$, and forms the convolution product $\mathcal{A}_{1} \star \mathcal{A}_{2}=m_{!}\left(\mathcal{A}_{1} \tilde{\otimes} \mathcal{A}_{2}\right)$. For simplicity, we sometimes denote $\left(\mathrm{P}_{L+G}\left(\mathrm{Gr}_{G}^{\mathrm{op}}\right), \star\right)$ by Sat ${ }_{G}^{\text {op }}$.

On the other hand, we have the following statements.

Lemma 2.22. There is an equivalence of the monoidal categories

$$
\mathrm{Id}^{\prime}: \mathrm{Sat}_{G}^{\mathrm{op}} \simeq \operatorname{Sat}_{G}
$$

sending the intersection cohomology sheaf $\mathrm{IC}_{\mu}^{\mathrm{op}}$ of $\mathrm{Gr}_{\leq \mu}^{\mathrm{op}}$ to $\mathrm{IC}_{\mu}$, where $\mathrm{Gr}_{\leq \mu}^{\mathrm{op}}$ is the closure of $\mathrm{Gr}_{\mu}^{\mathrm{op}}=L^{+} G \backslash L^{+} G \varpi^{\mu} L^{+} G$.

Let $(L G)_{\leq \mu}$ denote the preimage of $\mathrm{Gr}_{\leq \mu}$ under $L G \rightarrow \mathrm{Gr}$. Let $m$ be an integer large enough such that the $m$ th congruence subgroup $L^{+} G^{(m)} \subset L^{+} G \cap \varpi^{\mu} L^{+} G \varpi^{-\mu}$. Then we get the following diagram of surjective maps

$$
\mathrm{Gr}_{\leq \mu} \stackrel{\pi_{m}}{\leftarrow} \mathrm{Gr}_{\leq \mu}^{(m)}=(L G)_{\leq \mu} / L^{+} G^{(m)} \stackrel{\phi_{m}}{\rightarrow} L^{+} G \backslash(L G)_{\leq \mu}=\mathrm{Gr}_{\leq \mu}^{\mathrm{op}}
$$

Lemma 2.23. There exists a unique isomorphism of sheaves on $\operatorname{Gr}_{\leq \mu}^{(m)}$

$$
\mathrm{id}_{\mu}^{\prime}: \phi_{m}^{*} \mathrm{IC}_{\mu}^{\mathrm{op}} \simeq \pi_{m}^{*} \mathrm{IC}_{\mu}
$$

whose restriction to $\mathrm{Gr}_{\mu}^{(m)}$ is given by

$$
\left.\phi_{m}^{*} \mathrm{IC}_{\mu}^{\mathrm{op}}\right|_{\mathrm{Gr}_{\mu}^{(m)}}=\overline{\mathbb{Q}}_{\ell}[(2 \rho, \mu)]=\left.\pi_{m}^{*} \mathrm{IC}_{\mu}\right|_{\operatorname{Gr}_{\mu}^{(m)}} .
$$

In particular, $\phi_{m}^{*} \mathrm{IC}_{\mu}^{\mathrm{op}}[m \operatorname{dim} G]$ is perverse.

Remark 2.24. (i) Informally, one can think both categories as certain category of $\left(L^{+} G \times\right.$ $L^{+} G$ )-equivariant sheaves on $L G$. As we did not introduce sheaves on infinite-dimensional spaces, we give a concrete treatment here.

(ii) As $\pi_{m}$ is an $L^{m} G$-torsor, $\pi_{m}^{*}[m \operatorname{dim} G]$ preserves perversity. However, as we do not known whether $\phi_{m}$ is perfectly smooth, a priori it is not obvious that $\phi_{m}^{*} \mathrm{IC}_{\mu}^{\text {op }}[m \operatorname{dim} G]$ is perverse. On the other hand, as soon as the perversity of $\phi_{m}^{*} \mathrm{IC}_{\mu}^{\mathrm{op}}[m \operatorname{dim} G]$ is known, the existence and the uniqueness of $\mathrm{id}_{\mu}^{\prime}$ are clear.

Proof. We will prove these two lemmas simultaneously. First, if $\mu$ is minuscule, then $\mathrm{Gr}_{\leq \mu}$ and $\mathrm{Gr}_{\leq \mu}^{\mathrm{op}}$ are perfectly smooth so there is a unique isomorphism id ${ }_{\mu}^{\prime}: \phi_{m}^{*} \mathrm{IC}_{\mu}^{\mathrm{op}}=\overline{\mathbb{Q}}_{\ell}=\pi_{m}^{*} \mathrm{IC}_{\mu}$ as required by Lemma 2.23 .

If $\mu$ is quasi-minuscule, let $\widetilde{\mathrm{Gr}}_{\leq \mu} \rightarrow \mathrm{Gr}_{\leq \mu}$ denote the "resolution" as constructed in Lemma 2.12. We can form

$$
\widetilde{\mathrm{Gr}}_{\leq \mu}^{\mathrm{op}}=Q_{0} \backslash Q_{0} \times^{Q_{1 / 4}} Q_{1 / 2} \times^{Q_{3 / 4}} Q_{1}=Q_{1 / 4} \backslash Q_{1 / 2} \times{ }^{Q_{3 / 4}} Q_{1},
$$


and the map $\pi_{\mu}^{\mathrm{op}}: \widetilde{\mathrm{Gr}}_{\leq \mu}^{\mathrm{op}} \rightarrow \mathrm{Gr}_{\leq \mu}^{\mathrm{op}},\left(g, g^{\prime}\right) \mapsto g g^{\prime} \varpi^{\mu}$ is a "resolution" of $\mathrm{Gr}_{\leq \mu}^{\mathrm{op}}$. We define $\widetilde{\mathrm{Gr}}_{\leq \mu}^{(m)}$ by requiring that both squares in the diagram

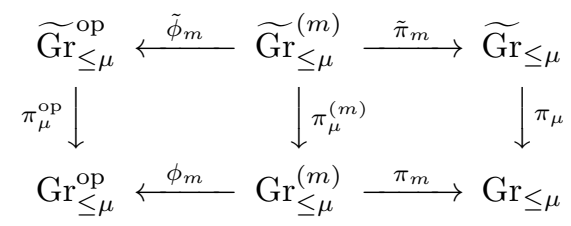

are Cartesian. Then we get the canonical isomorphisms

$$
\phi_{m}^{*} \mathrm{IC}_{\mu}^{\mathrm{op}} \oplus \phi_{m}^{*} \mathcal{C}^{\mathrm{op}} \simeq \phi_{m}^{*}\left(\pi_{\mu}^{\mathrm{op}}\right)_{*} \overline{\mathbb{Q}}_{\ell}[d] \simeq\left(\pi_{\mu}^{(m)}\right)_{*} \overline{\mathbb{Q}}_{\ell}[d] \simeq \pi_{m}^{*}\left(\pi_{\mu}\right)_{*} \overline{\mathbb{Q}}_{\ell}[d] \simeq \pi_{m}^{*} \mathrm{IC}_{\mu} \oplus \pi_{m}^{*} \mathcal{C},
$$

where $d=(2 \rho, \mu)$, and $\mathcal{C}$ and $\mathcal{C}^{\text {op }}$ are as in the proof of Lemma 2.11. We therefore obtain $\mathrm{id}_{\mu}^{\prime}$ as in Lemma 2.23 .

Now, let $\mu_{\bullet} \subset M$ as in $\$ 2.2 .3$ Let $\left(m_{1}, \ldots, m_{n}\right)$ be a sequence of integers, with $m_{n}$ large, and $m_{i}$ satisfying the conditions as in Lemma 2.17. Then from

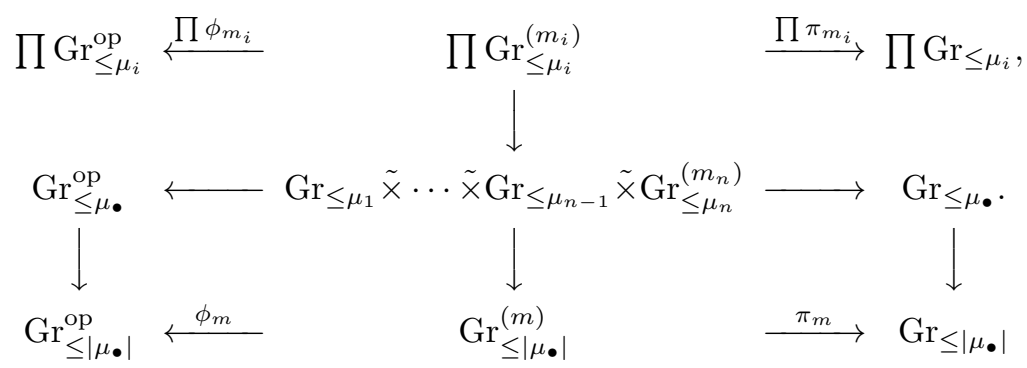

and the canonical isomorphisms $\prod_{i} \mathrm{id}_{\mu_{i}}^{\prime}:\left(\prod \phi_{m_{i}}\right)^{*}\left(\otimes \mathrm{IC}_{\mu_{i}}^{\mathrm{op}}\right) \simeq\left(\prod \pi_{m_{i}}\right)^{*}\left(\otimes \mathrm{IC}_{\mu_{i}}\right)$, we obtain a canonical isomorphism

$$
\operatorname{id}_{\mu_{\bullet}}^{\prime}: \phi_{m}^{*}\left(\mathrm{IC}_{\mu_{1}}^{\mathrm{op}} \star \cdots \star \mathrm{IC}_{\mu_{n}}^{\mathrm{op}}\right) \simeq \pi_{m}^{*}\left(\mathrm{IC}_{\mu_{1}} \star \cdots \star \mathrm{IC}_{\mu_{n}}\right) .
$$

By Lemma 2.16, we see that for every $\mu$ the isomorphism $\mathrm{id}_{\mu}^{\prime}$ as required in Lemma 2.23 exists.

In addition, this isomorphism also provides us the desired monoidal structure on $\mathrm{Id}^{\prime}$. Again, by Lemma 2.16, it is enough to exhibit the monoidal structure of $\mathrm{Id}^{\prime}$ when restricted to the subcategories $\operatorname{Id}^{\prime}: \operatorname{Sat}_{G}^{0, \text { op }} \simeq \operatorname{Sat}_{G}^{0}$, where Sat ${ }_{G}^{0}$ is defined before Lemma 2.16 and Sat ${ }_{G}^{0, \text { op }}$ is defined similarly. For $\lambda_{\bullet}, \mu_{\bullet} \subset M$, we write $\mathrm{IC}_{\lambda_{\bullet}}=\mathrm{IC}_{\lambda_{1}} \star \cdots \star \mathrm{IC}_{\lambda_{n}}$, etc. Then we get the canonical isomorphisms

$$
\operatorname{Hom}\left(\mathrm{IC}_{\lambda_{\bullet}}, \mathrm{IC}_{\mu_{\bullet}}\right) \simeq \operatorname{Hom}\left(\pi_{m}^{*} \mathrm{IC}_{\lambda_{\bullet}}, \pi_{m}^{*} \mathrm{IC}_{\mu_{\bullet}}\right) \simeq \operatorname{Hom}\left(\phi_{m}^{*} \mathrm{IC}_{\lambda_{\bullet}}^{\text {op }}, \phi_{m}^{*} \mathrm{IC}_{\mu_{\bullet}}^{\text {op }}\right) \simeq \operatorname{Hom}\left(\mathrm{IC}_{\lambda_{\bullet}}^{\text {op }}, \mathrm{IC}_{\mu_{\bullet}}^{\text {op }}\right),
$$

which is clearly independent of $m$ (as soon as $m$ large enough). This isomorphism provides the monoidal structure on $\operatorname{Id}^{\prime}$ as one can take $\mu_{\bullet}$ as the union of subsequences.

We have the following corollary of Lemma 2.23. For $\lambda \in \mathbb{X}_{\bullet}$, let $\mathcal{H}_{\lambda}^{j} \mathcal{A}$ (resp. $\mathcal{H}_{\lambda, !}^{j} \mathcal{A}$ denote the degree $j$ stallk (resp. costalk) cohomology of $\mathcal{A}$ at $\varpi^{\lambda}$.

Corollary 2.25. There is a canonical isomorphism $\mathcal{H}_{\lambda}^{j} \mathrm{id}: \mathcal{H}_{\lambda}^{j} \mathcal{A} \simeq \mathcal{H}_{\lambda}^{j} \operatorname{Id}^{\prime} \mathcal{A}$ for $\mathcal{A} \in$ $\mathrm{P}_{L^{+} G}\left(\mathrm{Gr}^{\mathrm{op}}\right)$, and similarly for $\mathcal{H}_{\lambda, \text { ! }}^{j}$

Proof. We prove the first half of the statement as the second half is obtained by Verdier duality. It is enough to assume that $\mathcal{A}=\mathrm{IC}_{\mu}^{\mathrm{op}}$. The isomorphism $\mathcal{H}_{\lambda}^{j} \mathrm{id}_{\mu}^{\prime}$ is given by the composition

$$
\mathcal{H}_{\lambda}^{j} \mathrm{IC}_{\mu}^{\mathrm{op}}=\mathcal{H}_{\lambda}^{j} \phi_{m}^{*} \mathrm{IC}_{\mu}^{\mathrm{op}} \stackrel{\mathcal{H}_{\lambda}^{j} \mathrm{id}_{\mu}^{\prime}}{\simeq} \mathcal{H}_{\lambda}^{j} \pi_{m}^{*} \mathrm{IC}_{\mu}=\mathcal{H}_{\lambda}^{j} \mathrm{IC}_{\mu}
$$

which is clearly independent of the choice of $m$. 
2.4.3. Now, we construct $c_{\mathcal{A}_{1}, \mathcal{A}_{2}}$ as in Theorem 2.20, In the equal characteristic situation, this is obtained from the interpretation of convolution products as fusion products ([MV] and [BD, §5.3]). It is unlikely that the fusion product exists in mixed characteristic. Our method is a kind of categorification of classical Gelfand's trick (see also [BD, §5.3.8] which modifies the construction of $\mathrm{Gi}]$ ).

Fix a pinning $(G, B, T, X)$ of $G$ and let $\theta^{\prime}$ be the involution that sends a dominant coweight $\lambda$ to its dual $\lambda^{*}=-w_{0}(\lambda)$, where $w_{0}$ is the longest element in the finite Weyl group. We define the anti involution $\theta$ of $G$ as $\theta(g)=\theta^{\prime}(g)^{-1}$. It induces the anti-involution of $L G$ and $L^{+} G$, still denoted by $\theta$, rather than $L \theta$ and $L^{+} \theta$, if no confusion will arise. Note that $\theta$ induces an isomorphism

$$
\theta: \mathrm{Gr}_{G}^{\mathrm{op}}=L^{+} G \backslash L G \simeq L G / L^{+} G=\mathrm{Gr}_{G},
$$

and therefore an equivalence of categories

$$
\theta^{*}: \mathrm{P}_{L+G}\left(\mathrm{Gr}_{G}\right) \simeq \mathrm{P}_{L+G}\left(\mathrm{Gr}_{G}^{\mathrm{op}}\right) .
$$

Now $\theta$ also induces

$$
\theta \tilde{\times} \theta: \mathrm{Gr}^{\mathrm{op}} \tilde{\times} \mathrm{Gr}^{\mathrm{op}} \rightarrow \mathrm{Gr} \tilde{\times} \mathrm{Gr}, \quad\left(g_{1}, g_{2}\right) \mapsto\left(\theta\left(g_{2}\right), \theta\left(g_{1}\right)\right),
$$

and there is a canonical isomorphism $(\theta \tilde{\times} \theta)^{*}\left(\mathcal{A}_{1} \tilde{\otimes} \mathcal{A}_{2}\right) \simeq \theta^{*} \mathcal{A}_{2} \tilde{\otimes} \theta^{*} \mathcal{A}_{1}$. Using $m(\theta \tilde{\times} \theta)=\theta m$, and the proper base change, there is a canonical isomorphism

$$
\theta^{*}\left(\mathcal{A}_{1} \star \mathcal{A}_{2}\right) \simeq \theta^{*} \mathcal{A}_{2} \star \theta^{*} \mathcal{A}_{1}
$$

Considering the 3 -fold convolutions, we see that $\theta^{*}$ is an anti-equivalence of monoidal categories.

Therefore, we get an anti-autoequivalence $\operatorname{Id}^{\prime} \circ \theta^{*}$ of $\operatorname{Sat}_{G}$ as a monoidal category. Now we define an isomorphism of (plain) functors

$$
e: \mathrm{Id}^{\prime} \circ \theta^{*} \rightarrow \mathrm{Id}
$$

We will fix a square root $\sqrt{-1}$ in $\overline{\mathbb{Q}}_{\ell}$ in the sequel and define $(-1)^{(\rho, \mu)}:=\sqrt{-1}^{(2 \rho, \mu)}$ for any coweight $\mu$. According to Lemma 2.1, it is enough to give an isomorphism $e_{\mu}: \mathrm{Id}^{\prime} \circ \theta^{*} \mathrm{IC}_{\mu} \rightarrow$ $\mathrm{IC}_{\mu}$ for every $\mu$. Note that $\theta^{*} \mathrm{IC}_{\mu}$ is (non-canonically) isomorphic to $\mathrm{IC}_{\mu}^{o p}$. We define the isomorphism $N_{\mu}: \theta^{*} \mathrm{IC}_{\mu} \rightarrow \mathrm{IC}_{\mu}^{o p}$ by requiring its restriction to $\mathrm{Gr}_{\mu}^{o p}$ is given by

$$
\left.\theta^{*} \mathrm{IC}_{\mu}\right|_{\operatorname{Gr}_{\mu}^{o p}}=\left.\mathrm{IC}_{\mu}\right|_{\mathrm{Gr}_{\mu}}=\overline{\mathbb{Q}}_{\ell}[(2 \rho, \mu)]=\overline{\mathbb{Q}}_{\ell}[(2 \rho, \mu)]=\left.\mathrm{IC}_{\mu}^{o p}\right|_{\operatorname{Gr}_{\mu}^{o p}}
$$

We define $M_{\mu}=(-1)^{-(\rho, \mu)} N_{\mu}$ and let $e_{\mu}=\operatorname{Id}^{\prime}\left(M_{\mu}\right)$. Let us emphasize that the factor $(-1)^{-(\rho, \mu)}$ is crucial.

Now, we define the isomorphism $c_{\mathcal{A}_{1}, \mathcal{A}_{2}}^{\prime}$ as

$c_{\mathcal{A}_{1}, \mathcal{A}_{2}}^{\prime}: \mathcal{A}_{1} \star \mathcal{A}_{2} \stackrel{e_{\mathcal{A}_{1} \star \mathcal{A}_{2}}}{\longleftarrow} \operatorname{Id}^{\prime} \theta^{*}\left(\mathcal{A}_{1} \star \mathcal{A}_{2}\right) \simeq \operatorname{Id}^{\prime}\left(\theta^{*} \mathcal{A}_{2} \star \theta^{*} \mathcal{A}_{1}\right) \simeq \operatorname{Id}^{\prime} \theta^{*} \mathcal{A}_{2} \star \operatorname{Id}^{\prime} \theta^{*} \mathcal{A}_{1} \stackrel{e_{\mathcal{A}_{2}} \star e_{\mathcal{A}_{1}}}{\longrightarrow} \mathcal{A}_{2} \star \mathcal{A}_{1}$.

Finally, the isomorphism $c_{\mathcal{A}_{1}, \mathcal{A}_{2}}$ is obtained by $c_{\mathcal{A}_{1}, \mathcal{A}_{2}}^{\prime}$ by a Koszul sign change (see also BD, §5.3.21] or [MV] after Remark 6.2 for a more elegant treatment). Namely, the category $\mathrm{P}_{L^{+} G}(\mathrm{Gr})$ admits a $\mathbb{Z} / 2$-grading induced by (1.5.3). We say $\mathcal{A}$ has pure parity if $p(\operatorname{Supp}(\mathcal{A}))$ is 1 or -1 , in which case we define $p(\mathcal{A})=p(\operatorname{Supp}(\mathcal{A}))$. Then

$$
c_{\mathcal{A}_{1}, \mathcal{A}_{2}}:=(-1)^{p\left(\mathcal{A}_{1}\right) p\left(\mathcal{A}_{2}\right)} c_{\mathcal{A}_{1}, \mathcal{A}_{2}}^{\prime}
$$

if $\mathcal{A}_{1}$ and $\mathcal{A}_{2}$ have the pure parity $p\left(\mathcal{A}_{1}\right)$ and $p\left(\mathcal{A}_{2}\right)$.

Remark 2.26. We can give a more concrete description of the commutativity constraints. For this, let us assume that $\mathcal{A}_{1}=\mathrm{IC}_{\mu}$ and $\mathcal{A}_{2}=\mathrm{IC}_{\nu}$. We write $\mathrm{IC}_{\mu} \star \mathrm{IC}_{\nu}=\bigoplus_{\lambda} \mathrm{IC}_{\lambda} \otimes V_{\mu, \nu}^{\lambda}$, where $V_{\mu, \nu}^{\lambda}=\operatorname{Hom}\left(\mathrm{IC}_{\lambda}, \mathrm{IC}_{\mu}, \mathrm{IC}_{\nu}\right)$. So $c_{\mathcal{A}_{1}, \mathcal{A}_{2}}$ is given by a collection of isomorphisms $c_{\mu, \nu}^{\lambda}$ : $V_{\mu, \nu}^{\lambda} \simeq V_{\nu, \mu}^{\lambda}$ 
By Remark 2.4 there is a canonical basis of $V_{\mu, \nu}^{\lambda}$ given by the set $\mathbb{B}_{\mu, \nu}^{\lambda}$ of irreducible components of $\operatorname{Gr}_{\mu, \nu} \cap m^{-1}\left(\operatorname{Gr}_{\lambda}\right)$ of dimension $(\rho, \mu+\nu+\lambda)$. Note that there is a canonical bijection between

$$
\mathbf{b}_{\mu, \nu}^{\lambda}: \mathbb{B}_{\mu, \nu}^{\lambda} \simeq \mathbb{B}_{\nu, \mu}^{\lambda} .
$$

Indeed, $\theta$ induces an isomorphism between $\left(\mathrm{Gr}_{\mu} \tilde{\times} \mathrm{Gr}_{\nu}\right) \cap m^{-1}\left(\mathrm{Gr}_{\lambda}\right)$ and $\left(\mathrm{Gr}_{\nu}^{\mathrm{op}} \tilde{\times} \mathrm{Gr}_{\mu}^{\mathrm{op}}\right) \cap$ $m^{-1}\left(\mathrm{Gr}_{\lambda}^{\mathrm{op}}\right)$. The latter is stable under the right translation by $L^{+} G$ and there is a canonical isomorphism (in whatever sense) $\left(\left(\mathrm{Gr}_{\nu}^{\mathrm{op}} \tilde{\times} \mathrm{Gr}_{\mu}^{\mathrm{op}}\right) \cap m^{-1}\left(\mathrm{Gr}_{\lambda}^{\mathrm{op}}\right)\right) / L^{+} G=L^{+} G \backslash\left(\left(\mathrm{Gr}_{\nu} \tilde{\times} \mathrm{Gr}_{\mu}\right) \cap\right.$ $\left.m^{-1}\left(\operatorname{Gr}_{\lambda}\right)\right)$. These two isomorphisms together induces $\mathbf{b}_{\mu, \nu}^{\lambda}$. Then from the construction

$$
c_{\mu, \nu}^{\lambda}=(-1)^{(\rho, \mu+\nu-\lambda)+(2 \rho, \mu)(2 \rho, \nu)} \mathbf{b}_{\mu, \nu}^{\lambda} .
$$

For example, if $G=\mathrm{GL}_{n}, \mu=\omega_{1}$ and $\nu=\omega_{1}^{*}$, then $c_{\mu, \nu}^{0}=1$, and $c_{\mu, \nu}^{\mu+\nu}=(-1)^{n-1}$.

2.4.4. We prove that $c_{\mathcal{A}_{1}, \mathcal{A}_{2}}$ constructed as above satisfies the requirement as in Proposition 2.20. In fact, it is easy to see from the definition that Proposition 2.20 is the consequence of the following three statements Lemma 2.27-Lemma 2.29

To state the first lemma, note that Lemma 2.17 and Lemma 2.18 hold for Sat ${ }_{G}^{\mathrm{op}}$, and therefore $\mathrm{H}^{*}:$ Sat $^{\mathrm{op}} \rightarrow$ Vect $_{\overline{\mathbb{Q}}_{\ell}}$ has a natural monoidal structure.

Lemma 2.27. There is a natural isomorphism of monoidal functors $\gamma: \mathrm{H}^{*} \simeq \mathrm{H}^{*} \circ \mathrm{Id}^{\prime}$ : $\mathrm{Sat}_{G}^{\mathrm{op}} \rightarrow \operatorname{Vect}_{\overline{\mathbb{Q}}_{\ell}}$.

Proof. It is enough to construct the canonical isomorphism $\gamma_{\mu}: \operatorname{IH}^{*}\left(\operatorname{Gr}_{\leq \mu}^{\text {op }}\right) \simeq \operatorname{IH}^{*}\left(\operatorname{Gr}_{\leq \mu}\right)$ for every $\mu$. From the diagram (2.4.1), we get a canonical isomorphism

$$
\mathrm{IH}_{L^{+} G}^{*}\left(\mathrm{Gr}_{\leq \mu}^{\mathrm{op}}\right) \simeq \mathrm{IH}_{L^{+} G \times L^{+} G}^{*}\left(\mathrm{Gr}_{\leq \mu}^{(m)}\right) \simeq \mathrm{IH}_{L^{+} G}^{*}\left(\mathrm{Gr}_{\leq \mu}\right)
$$

as $\left(R_{\bar{G}, \ell} \otimes R_{\bar{G}, \ell}\right)$-bimodules. Note that this is independent of the choice of $m$ (as soon as it is large). As

$$
\mathrm{IH}^{*}\left(\mathrm{Gr}_{\leq \mu}\right):=\overline{\mathbb{Q}}_{\ell} \otimes_{R_{\bar{G}, \ell}} \mathrm{IH}_{L+G}^{*}\left(\mathrm{Gr}_{\leq \mu}\right), \quad \mathrm{IH}^{*}\left(\mathrm{Gr}_{\leq \mu}^{\mathrm{op}}\right):=\operatorname{IH}_{L+G}^{*}\left(\mathrm{Gr}_{\leq \mu}^{\mathrm{op}}\right) \otimes_{R_{\bar{G}, \ell}} \overline{\mathbb{Q}}_{\ell},
$$

we get the desired isomorphism $\gamma_{\mu}$ by Lemma 2.18. It is clear from the construction of the monoidal structure of $\mathrm{H}^{*}$ given by Lemma 2.17 and Lemma 2.18 and the construction of the monoidal structure on $\mathrm{Id}^{\prime}$ as in $\$ 2.4 .2$ that $\gamma$ is an isomorphism of monoidal functors.

The second lemma is

Lemma 2.28. There is a canonical isomorphism of functors $\delta: \mathrm{H}^{*} \simeq \mathrm{H}^{*} \circ \theta^{*}$, such that for every $\mathcal{A}_{1}, \mathcal{A}_{2} \in \mathrm{P}_{L^{+} G}(\mathrm{Gr})$, the following diagram is commutative.

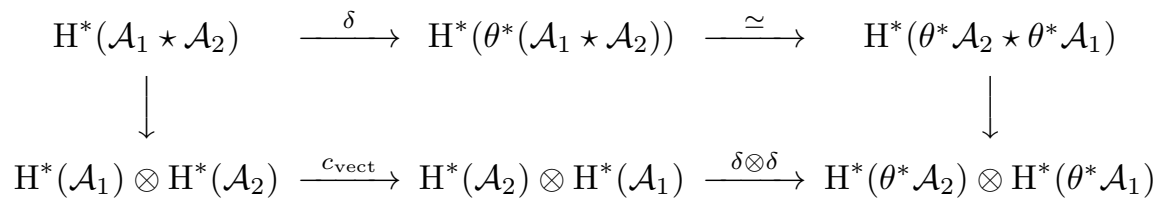

Proof. If $f: X \rightarrow Y$ is a morphism and $\mathcal{F}$ a complex of sheaves on $Y$, there is a canonical map $f^{*}: \mathrm{H}^{*}(Y, \mathcal{F}) \rightarrow \mathrm{H}^{*}\left(Y, f_{*} f^{*} \mathcal{F}\right) \simeq \mathrm{H}^{*}\left(X, f^{*} \mathcal{F}\right)$. Applying this to $\theta: \mathrm{Gr}^{\text {op }} \simeq$ Gr gives the isomorphism $\delta$.

As before, for a closed subset $Z \subset \mathrm{Gr}^{\text {op }}$, let $Z^{(m)}$ denote its preimage in $L^{+} G^{(m)} \backslash L G \rightarrow$ $\mathrm{Gr}^{\mathrm{op}}$. Note that in the notation as in Lemma 2.17, the following diagram is commutative

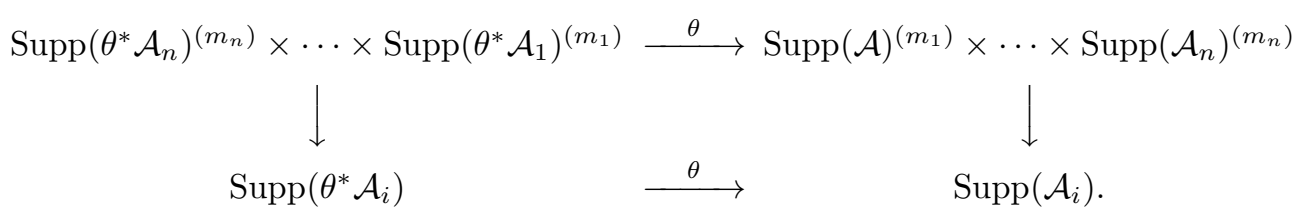


Then from the construction of the isomorphism in Lemma 2.17, the following diagram is commutative

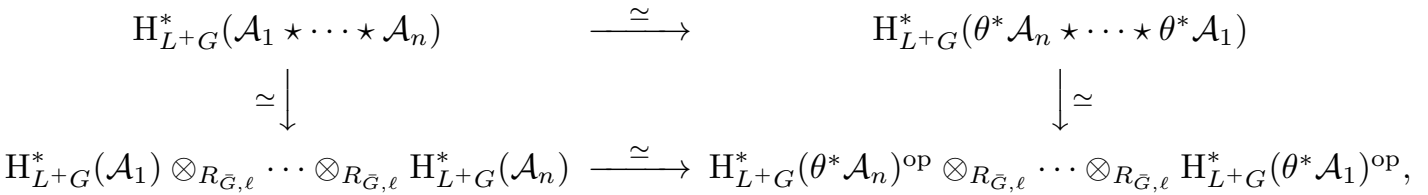
where for an $R_{\bar{G}, \ell^{-b i m o d u l e}} M, M^{\text {op }}$ denotes the new $R_{\bar{G}, \ell^{-}}$-bimodule structure on $M$ by switching the two factors. After specialization $R_{\bar{G}, \ell} \rightarrow \overline{\mathbb{Q}}_{\ell}$, we see that $\delta$ is an isomorphism of monoidal functors.

For every $\mathcal{A} \in \mathrm{P}_{L^{+} G}(\mathrm{Gr})$, we define an automorphism of its cohomology

$$
\Theta: \mathrm{H}^{*}(\mathcal{A}) \stackrel{\delta}{\simeq} \mathrm{H}^{*}\left(\theta^{*} \mathcal{A}\right) \stackrel{\mathcal{\gamma}}{\simeq} \mathrm{H}^{*}\left(\operatorname{Id}^{\prime} \circ \theta^{*} \mathcal{A}\right) \stackrel{\mathrm{H}^{*}(e)}{\simeq} \mathrm{H}^{*}(\mathcal{A}) .
$$

The above two lemmas imply that the following diagram

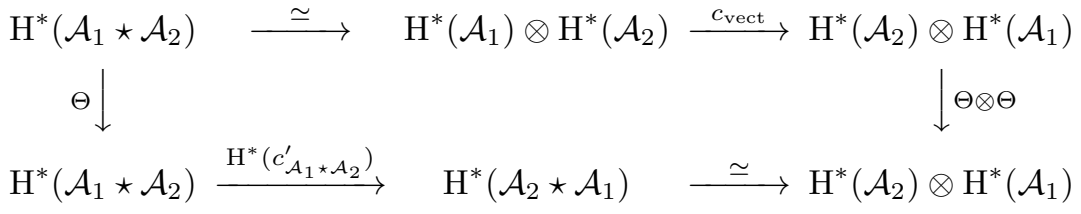

is commutative. Now it is easy to see that Proposition 2.20 follows from the following lemma.

Lemma 2.29. The map $\Theta$ acts on $\mathrm{H}^{j}(\mathcal{A})$ by multiplication by $\sqrt{-1}^{j}$.

Note that by the definition of $e$, and our normalization $\operatorname{IH}^{*}\left(\mathrm{Gr}_{\leq \mu}\right)=\mathrm{H}^{*}\left(\mathrm{IC}_{\mu}[-(2 \rho, \mu)]\right)$, it is enough to show that

Lemma 2.30. The map $\Theta_{\mu}: \mathrm{IH}^{2 j}\left(\mathrm{Gr}_{\leq \mu}\right) \stackrel{\mathrm{H}\left(N_{\mu}\right) \delta}{\simeq} \mathrm{IH}^{2 j}\left(\mathrm{Gr}_{\leq \mu}^{\mathrm{op}}\right) \stackrel{\mathcal{\gamma}}{\simeq} \mathrm{IH}^{2 j}\left(\mathrm{Gr}_{\leq \mu}\right)$ is multiplication by $(-1)^{j}$, where $N_{\mu}: \theta^{*} \mathrm{IC}_{\mu} \rightarrow \mathrm{IC}_{\mu}^{\text {op }}$ is the canonical isomorphism introduced in 2.4 .3 .

We do not know a direct proof of this lemma. In [LY], its equal characteristic analogue was deduced from the equal characteristic geometric Satake. They use this formula to deduce a combinatoric formula for the affine Hecke algebra, as conjectured by Lusztig Lu2. We will reverse their steps to deduce this lemma from this combinatoric formula. In the sequel, we follow the convention in literature to write $\mathrm{H}\left(N_{\mu}\right) \delta$ as $\theta^{*}$. It should not be confused with the pullback of sheaves. First note that

Lemma 2.31. The map $\Theta_{\mu}$ is an involution.

Proof. Choose some $m, m^{\prime}$, such that the following diagram is commutative

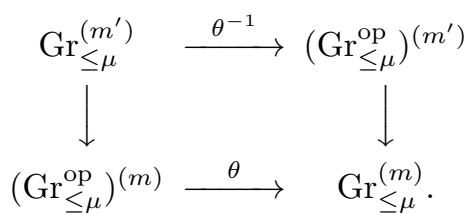

Then taking $\left(L^{+} G \times L^{+} G\right)$-equivariant intersection cohomology and specializing $R_{\bar{G}, \ell} \rightarrow \overline{\mathbb{Q}}_{\ell}$, implies the commutativity of the following diagram

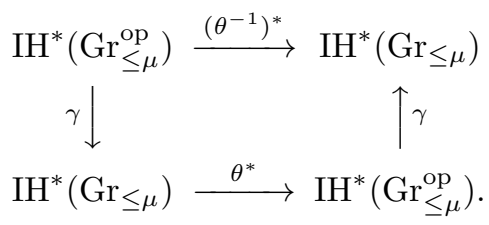

The lemma follows. 
To continue, let us understand a toy case. Note that $\theta$ induces an isomorphism between $L^{+} G$-orbits $\mathrm{Gr}_{\mu}^{\text {op }} \simeq \mathrm{Gr}_{\mu}$, and therefore we have a canonical isomorphism

$$
\stackrel{\circ}{\Theta}_{\mu}: \mathrm{H}^{*}\left(\mathrm{Gr}_{\mu}\right) \stackrel{\theta^{*}}{\simeq} \mathrm{H}^{*}\left(\mathrm{Gr}_{\mu}^{\mathrm{op}}\right) \stackrel{\gamma}{\simeq} \mathrm{H}^{*}\left(\mathrm{Gr}_{\mu}\right),
$$

where the isomorphisms $\gamma$ is constructed by the same way as in Lemma 2.27

Lemma 2.32. The map $\stackrel{\circ}{\Theta}_{\mu}$ acts as $(-1)^{j}$ on $\mathrm{H}^{2 j}\left(\mathrm{Gr}_{\mu}\right)$.

Proof. The argument is essentially the same as LY, Lemma 3.3], although the set-up is different (the authors of loc. cit. works over $\mathbb{C}$ and with the based loop group of a compact Lie group rather that the affine Grassmannian). Note that there is a canonical projection

$$
\mathrm{Gr}_{\mu} \simeq L^{+} G /\left(L^{+} G \cap \varpi^{\mu} L^{+} G \varpi^{-\mu}\right) \rightarrow\left(\bar{G} / \bar{P}_{-\mu}\right)^{p^{-\infty}}, \quad g \varpi^{\mu} \mapsto g \bmod \varpi
$$

where $\bar{P}_{-\mu}$, as in Lemma 2.11, is the parabolic subgroup of $\bar{G}$ whose roots are those $\alpha$ with $\langle\alpha, \mu\rangle \leq 0$. As the fibers are perfection of affine spaces of the same dimension, we get a canonical ring isomorphism $\mathrm{H}^{*}\left(\bar{G} / \bar{P}_{-\mu}\right) \simeq \mathrm{H}^{*}\left(\mathrm{Gr}_{\mu}\right)$. Therefore, $\mathrm{H}^{*}\left(\mathrm{Gr}_{\mu}\right)$ is generated by $\mathrm{H}^{2}$. In addition, it is clear that $\stackrel{\Theta}{\mu}_{\mu}$ is a ring homomorphism so it is enough to prove that $\stackrel{\circ}{\Theta}_{\mu}$ is $(-1)$ on $\mathrm{H}^{2}$.

On the other hand $\mathrm{Gr}_{\mu}^{\text {op }}$ projects to $\left(\bar{G} / \bar{P}_{\mu}\right)^{p^{-\infty}}$ given by $\varpi^{\mu} g \mapsto g^{-1} \bmod \varpi$. A direct computation shows that the following diagram is commutative

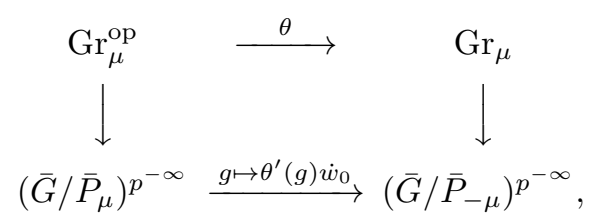

where $\dot{w}_{0}$ is a lifting of $w_{0}$ to $\bar{G}$. Taking the equivariant cohomology, we see the following diagram is commutative

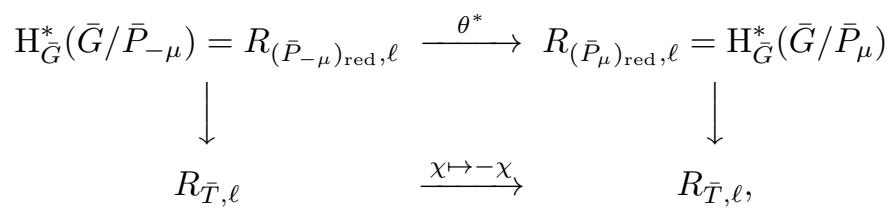

where $\chi \in \mathbb{X}^{\bullet}(\bar{T})$, regarded as elements in $R_{\bar{T}, \ell}$ of degree two.

On the other hand, the isomorphism $\mathrm{H}_{\bar{G}}^{*}\left(\bar{G} / \bar{P}_{\mu}\right) \simeq \mathrm{H}_{\bar{G}}^{*}\left(\bar{G} / \bar{P}_{-\mu}\right)$, given by $\gamma: \mathrm{H}_{L^{+}{ }_{G}}^{*}\left(\operatorname{Gr}_{\mu}^{\text {op }}\right) \simeq$

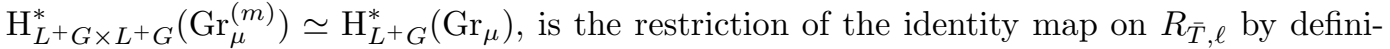
tion. Therefore, the equivariant version of $\stackrel{\circ}{\Theta}_{\mu}$ acts as $(-1)$ on degree two parts. Specializing gives the lemma.

Remark 2.33. This in particular proves Lemma2.30 in the case $\mu$ is minuscule. The difficulty to prove this lemma for general $\mu$ is that the intersection cohomology ring is not generated by Chern classes but we do not know more cohomology classes in it 12 .

To continue, it is convenient to set $\mathbf{C}_{\mu}=\mathrm{IC}_{\mu}[(2 \rho, \mu)]$, as in [LY]. Then for each $\lambda \leq \mu$, let $i_{\lambda}: \mathrm{Gr}_{\lambda} \rightarrow \mathrm{Gr}_{\leq \mu}$ denote the corresponding locally closed embedding. For $j$, let $\mathcal{H}_{\lambda}^{j} \mathbf{C}_{\mu}$ denote the degree $j$ sheaf cohomology of $i_{\lambda}^{*} \mathbf{C}_{\mu}$, which is a constant sheaf on $\mathrm{Gr}_{\lambda}$. Then there is a canonical isomorphism

$$
\mathcal{H}_{\lambda}^{j} \Psi_{\mu}: \mathcal{H}_{\lambda}^{j} \mathbf{C}_{\mu}=\mathcal{H}_{\lambda}^{j} \theta^{*} \mathbf{C}_{\mu} \simeq \mathcal{H}_{\lambda}^{j} \mathbf{C}_{\mu}^{\mathrm{op}} \simeq \mathcal{H}_{\lambda}^{j} \mathbf{C}_{\mu}
$$

where the third isomorphism is $N_{\mu}: \theta^{*} \mathrm{IC}_{\mu} \simeq \mathrm{IC}_{\mu}^{\text {op }}$, and the last isomorphism is from Corollary 2.25. Clearly, $\mathcal{H}_{\lambda}^{j} \Psi_{\mu}$ is an involution. Recall that the existence of Demazure

\footnotetext{
${ }^{12}$ Although there are MV basis in $\mathrm{IH}^{*}\left(\mathrm{Gr}_{\leq \mu}\right)$, it seems hard to understand the map $\gamma$ in terms of them.
} 
"resolution" in our setting implies that all the stalk cohomology of $\mathbf{C}_{\mu}$ concentrate on even degrees.

Lemma 2.34. The action $\mathcal{H}_{\lambda}^{2 j} \Psi_{\mu}$ is multiplication by $(-1)^{j}$.

Now, we prove Lemma 2.30, assuming this Lemma. In [LY, 3.4, 6.4], it is shown that equal characteristic analogue of Lemma 2.30 implies equal characteristic analogue of Lemma 2.34. But their argument can be reversed. We sketch it here and refer to loc. cit. for details (again, their set-up is different). We extend the partial order " $\leq$ " on $\mathbb{X}_{0}^{+}$to a total order, still denoted by $\leq$. We consider the stratification of $\mathrm{Gr}_{\leq \mu}$ given by $\left\{\mathrm{Gr}_{\lambda}, \lambda \leq \mu\right\}$. Let $\mathrm{Gr}_{<\lambda}=\sqcup_{\lambda^{\prime}<\lambda} \mathrm{Gr}_{\lambda^{\prime}}$ and let $i_{<\lambda}$ and $i_{\leq \lambda}$ denote the corresponding closed embeddings from $\mathrm{Gr}_{<\lambda}$ and $\mathrm{Gr}_{\leq \lambda}$ to $\mathrm{Gr}_{\leq \mu}$. Then there is the long exact sequence of cohomology

$$
\cdots \rightarrow \mathrm{H}^{i}\left(\mathrm{Gr}_{<\lambda}, i_{<\lambda}^{!} \mathbf{C}_{\mu}\right) \rightarrow \mathrm{H}^{i}\left(\mathrm{Gr}_{\leq \lambda}, i_{\leq \lambda}^{!} \mathbf{C}_{\mu}\right) \rightarrow \mathrm{H}^{i}\left(\mathrm{Gr}_{\lambda}, i_{\lambda}^{!} \mathbf{C}_{\mu}\right) \rightarrow \cdots
$$

which splits into short exact sequences as all the cohomology in odd degree vanish. Therefore, we get a filtration on $\operatorname{IH}^{*}\left(\mathrm{Gr}_{\leq \mu}\right)$, given by $\operatorname{Im}\left(\mathrm{H}^{i}\left(\mathrm{Gr}_{\leq \lambda}, i_{\leq \lambda}^{!} \mathbf{C}_{\mu}\right) \rightarrow \mathrm{IH}^{*}\left(\mathrm{Gr}_{\leq \mu}\right)\right)$. The associated graded are $\oplus_{\lambda \leq \mu} \mathrm{H}^{i}\left(\mathrm{Gr}_{\lambda}, i_{\lambda}^{!} \mathbf{C}_{\mu}\right)$. There is a similar picture on $\mathrm{Gr}_{\leq \mu}^{\mathrm{op}}$.

The isomorphisms $\theta^{*}: \operatorname{IH}^{*}\left(\mathrm{Gr}_{\leq \mu}\right) \simeq \operatorname{IH}^{*}\left(\mathrm{Gr}_{\leq \mu}^{\text {op }}\right)$ and $\gamma: \operatorname{IH}^{*}\left(\mathrm{Gr}_{\leq \mu}\right) \simeq \operatorname{IH}^{*}\left(\operatorname{Gr}_{\leq \mu}\right)$ preserve the filtrations on $\mathrm{IH}^{*}\left(\mathrm{Gr}_{\leq \mu}\right)$ and on $\mathrm{IH}^{*}\left(\mathrm{Gr}_{\leq \mu}^{\mathrm{op}}\right)$, and therefore gives rise to isomorphisms

$$
\operatorname{gr} \Theta: \operatorname{gr~IH}\left(\mathrm{Gr}_{\leq \mu}\right) \stackrel{\operatorname{gr} \theta^{*}}{\simeq} \operatorname{gr~IH}\left(\mathrm{Gr}_{\leq \mu}^{\mathrm{op}}\right) \stackrel{\operatorname{gr} \gamma}{\rightarrow} \operatorname{gr~IH}\left(\mathrm{Gr}_{\leq \mu}\right)
$$

Note that $i_{\lambda} \mathbf{C}_{\mu}=\left(i_{\lambda}^{*} \mathbf{C}_{\mu}[2(2 \rho, \lambda-\mu)]\right)^{*}$, and it is easy to identify $\operatorname{gr} \Theta$ with the direct sum over $\lambda$ of the maps

$$
\stackrel{\circ}{\Theta}_{\lambda} \otimes \mathcal{H}_{\lambda, !}^{*} \Psi_{\mu}: \mathrm{H}^{*}\left(\mathrm{Gr}_{\lambda}\right) \otimes i_{\lambda}^{!} \mathbf{C}_{\mu} \simeq \mathrm{H}^{*}\left(\mathrm{Gr}_{\lambda}\right) \otimes i_{\lambda}^{!} \mathbf{C}_{\mu},
$$

where $\mathcal{H}_{\lambda, !}^{*} \Psi_{\mu}$ is the inverse of the dual of $\mathcal{H}_{\lambda}^{*} \Psi_{\mu}$.

So the action of $\operatorname{gr} \Theta$ on degree $2 j$ piece of $\mathrm{H}^{*}\left(\mathrm{Gr}_{\lambda}\right) \otimes \mathcal{H}_{\lambda}^{*} \mathbf{C}_{\mu}$ is given by $(-1)^{j}$. But as $\Theta$ itself is an involution, it acts on $\operatorname{IH}^{2 j}\left(\mathrm{Gr}_{\leq \mu}\right)$ by $(-1)^{j}$.

2.4.5. It remains to prove Lemma 2.34 Recall the notations as in 1.5 .2 . We fix the apartment $A(G, F)$ corresponding to $T$, a hyperspecial vertex $v_{0}$ corresponding to $L^{+} G$, and an alcove a corresponding to the Iwahori $I$ whose reduction $\bmod \varpi$ is $\bar{B} \subset \bar{G}$. Let $\widetilde{W}$ denote the Iwahori-Hecke algebra of $G(F)$, and $W_{a} \subset \widetilde{W}$ denotes the corresponding affine Hecke algebra, with the set of simple reflections $s_{i}, i \in \mathbb{S}$ determined by a. Let $s_{0}$ be the simple reflection corresponding $v_{0}$. Let $J=\mathbb{S}-\{0\}$, and $W_{J} \subset \widetilde{W}_{a}$ denote the Weyl group for $G / \mathcal{O}$. Let $w_{J}$ denote the longest element in $W_{J}$. As $v_{0}$ is hyperspecial, $W_{J}$ is isomorphic to the finite Weyl group $\bar{W}:=\widetilde{W} / \mathbb{X}$ • of $G$, and $w_{J}$ maps to the longest element $w_{0}$ in $\bar{W}$ mentioned before. Let $\Omega \subset \widetilde{W}$ denote the length zero element, i.e. those that fix the alcove. Then it acts on $W_{a}$ by conjugation and $\widetilde{W}=W_{a} \rtimes \Omega$. Let $*: \widetilde{W} \rightarrow \widetilde{W}$ be the involution given by $w^{*}:=w_{J} w w_{J}$ for $w \in W_{J}$ and $\lambda^{*}=-w_{0}(\lambda)$ for $\lambda \in \mathbb{X}_{\bullet}$. This is an involution of $\widetilde{W}$ which stabilizes $\left\{s_{i}, i \in \mathbb{S}\right\}$ and fixes $s_{0}$.

Let $\omega \in \Omega$. Then by [LY, Lemma 6.2], $\omega^{*}=\omega^{-1}$, and

$$
\diamond: W_{a} \rightarrow W_{a}, \quad w \mapsto w^{\diamond}:=\omega w^{*} \omega^{-1},
$$

is an involution of $W_{a}$, which stabilizes $\left\{s_{i}, i \in \mathbb{S}\right\}$. Let $I_{\diamond}=\left\{w \in W_{a} \mid w^{\diamond}=w^{-1}\right\}$, and $W_{J}^{\diamond}=\left\{w^{\diamond} \mid w \in W_{J}\right\}=\omega W_{J} \omega^{-1}$. Then as argued in [Lu2, Proposition 8.2] and [LY, Theorem $6.3(1)]$, the longest element in every $W_{J} \times W_{J}^{\diamond}$-double coset belongs to $I_{\diamond}$.

Applying the results of [LV] $\mathrm{Lu2}$ to $\left(W_{a},\left\{s_{i}, i \in \mathbb{S}\right\}, \diamond\right)$, one attaches a polynomial $P_{y, w}^{\sigma, \diamond}(q) \in \mathbb{Z}[q]$ to every $y, w \in I_{\diamond}$, with $y \leq w$. On the other hand, there is the usual Kazhdan-Lusztig polynomial $P_{y, w}(q)$ attached to $y, w$ [KL1]. The following theorem is conjectured in [Lu2, Conjecture 8.4], and is proved in [LY, Theorem 6.3]. 
Theorem 2.35. Let $d_{1}$ and $d_{2}$ be longest elements of $\left(W_{J}, W_{J}^{\diamond}\right)$-double cosets in $W_{a}$. Then

$$
P_{d_{1}, d_{2}}^{\sigma, \diamond}(q)=P_{d_{1}, d_{2}}(-q) \text {. }
$$

Let us note that this theorem is deduced in $[\mathrm{LY}]$ from equal characteristic analogue of Lemma 2.32.

Finally, we explain why Lemma 2.34 follows from this theorem. Let $\mu \in \mathbb{X}$ •, and let $\omega$ denote the unique element in $\Omega$ such that $\varpi^{\mu} \in W_{a} \omega$. Let $d_{\mu}$ be the longest element in $W_{J} \varpi^{\mu} W_{J} \omega^{-1}=W_{J}\left(\varpi^{\mu} \omega^{-1}\right) W_{J}^{\diamond}$. Then for $\lambda \leq \mu, \varpi^{\lambda} \omega^{-1} \in W_{a}$ and let $d_{\lambda}$ denote the corresponding longest element in $W_{J} \varpi^{\lambda} \omega^{-1} W_{J}^{\diamond}$. It is clear that the usual Kazhdan-Lusztig theory [KL1, KL2] works in our situation as well, so $P_{y, w}(q)$ is the Poincare polynomial for the stalk cohomology at $y$ of the intersection cohomology sheaf $\mathrm{IC}_{w}$ of the Schubert variety $S_{w}$ on the affine flag variety $\mathcal{F} \ell=L G / I$. Then in particular (see [Lu1]),

$$
P_{d_{\lambda}, d_{\mu}}(q)=\sum\left(\operatorname{dim} \mathcal{H}_{\lambda}^{2 j} \mathbf{C}_{\mu}\right) q^{j} .
$$

On the other hand, in [LV, §3], similar interpretations are given to the polynomials $P_{y, w}^{\sigma, \diamond}$. Such interpretation will in particular implies that

$$
P_{d_{\lambda}, d_{\mu}}^{\sigma, \diamond}(q)=\sum \operatorname{tr}\left(\mathcal{H}_{\lambda}^{2 j} \Psi_{\mu} \mid \mathcal{H}_{\lambda}^{2 j} \mathbf{C}_{\mu}\right) q^{j}
$$

Since $\mathcal{H}_{\lambda}^{2 j} \Psi_{\mu}$ is an involution, Theorem 2.35 implies Lemma 2.34 .

2.5. Conclusion. We have endowed $\mathrm{P}_{L^{+} G}(\mathrm{Gr})$ with a symmetric monoidal category structure, and endowed the hypercohomology functor $\mathrm{H}^{*}: \mathrm{P}_{L^{+} G}(\mathrm{Gr}) \rightarrow$ Vect $_{\overline{\mathbb{Q}_{\ell}}}$ a tensor functor structure. It is clear that $\mathrm{IC}_{0}$ is a unit object in $\mathrm{P}_{L^{+} G}(\mathrm{Gr})$. Now we proceed as in $\mathrm{MV}$, $\S 7$ to conclude that $\left(\mathrm{P}_{L^{+} G}, \star, \mathrm{H}^{*}\right)$ is a Tannakian category with the fiber functor $\mathrm{H}^{*}$. Let $\tilde{G}=$ Aut $^{\otimes} \mathrm{H}^{*}$ denote the Tannakian group. Then it is a connected reductive group, as argued in [MV, §7]. With some effort as in [MV, §7], one can endow $\tilde{G}$ with a pinning and identified the based root datum of $\tilde{G}$ dual to the root datum of $G$, and therefore $\tilde{G}$ is identified with the dual group $\hat{G}$ of $G$. Another quicker (but less canonical) identification can be achieved as follows: there are isomorphisms at the level of $K$-rings

$$
K\left(\operatorname{Rep}_{\overline{\mathbb{Q}}_{\ell}}(\tilde{G})\right) \simeq K\left(\mathrm{P}_{L^{+} G}(\mathrm{Gr})\right) \simeq K\left(\operatorname{Rep}_{\overline{\mathbb{Q}}_{\ell}}(\hat{G})\right),
$$

where the first isomorphism is by definition and the second isomorphism is the classical Satake isomorphism. Then $\tilde{G} \simeq \hat{G}$ as both groups are reductive. See Ri] for more details.

\section{Dimension of affine Deligne-Lusztig varieties}

As explained in the introduction, one of the motivations to construct mixed characteristic affine Grassmannians is to study the Rapoport-Zink (RZ) spaces. Here we give one application. More applications will appear in [XZ].

\subsection{Dimension of affine Deligne-Lusztig varieties.}

3.1.1. Let $F$ be a non-archimedean local field with ring of integers $\mathcal{O}$, and let $L$ be the completion of its maximal unramified extension, with $\mathcal{O}_{L}$ its ring of integers. Let $\sigma \in$ $\operatorname{Gal}(L / F)$ denote the Frobenius element. Let $G$ be an unramfied group over $F$. For $b \in G(L)$ and $\mu \in \mathbb{X}_{\bullet}^{+}$, we define the (closed) affine Deligne-Lusztig "variety" as

$$
X_{\leq \mu}(b)=\left\{g \bmod L^{+} G \in \operatorname{Gr}_{G} \mid g^{-1} b \sigma(g) \in \overline{L^{+} G \varpi^{\mu} L^{+} G}\right\},
$$

which is closed in $\operatorname{Gr}_{G}$. More precisely, one can interpret $X_{\leq \mu}(b)$ as the following moduli functor: Let $\mathcal{E}_{0}$ be the trivial $G$-torsor on $\mathcal{O}$, with an isomorphism $b: \sigma^{*} \mathcal{E}_{0} \otimes F \rightarrow \mathcal{E}_{0} \otimes F$. Then for a perfect $k$-algebra $R$,

$$
X_{\leq \mu}(b)(R)=\left\{(\mathcal{E}, \beta) \mid \operatorname{Inv}_{x}\left(\beta^{-1} b \sigma(\beta)\right) \leq \mu, \forall x \in \operatorname{Spec} R\right\} .
$$


By Lemma 1.7 (and Tannakian considerations), $X_{\leq \mu}(b)$ is a closed subset of $\operatorname{Gr}_{G}$. One can replace " $\leq$ " in the above definition by "=", which defines an open subset of $X_{\leq \mu}(b)$, denoted by $X_{\mu}(b)$. If we denote $\Phi=\beta^{-1} b \sigma(\beta)$, then $(\mathcal{E}, \Phi)$ is an F-crystal with $G$-structure on $\operatorname{Spec} R$, whose Hodge polygon is bounded by $\mu$ (resp. equal to $\mu$ ).

It turns out its dimension is finite, and Rapoport gave a conjecture of its dimension $([\underline{\mathrm{R}})$ with a reformulation given by Kottwitz ( $\mathrm{GHKR}]$ )

$$
\operatorname{dim} X_{\leq \mu}(b)=\left\langle\rho, \mu-\nu_{b}\right\rangle-\frac{1}{2} \operatorname{def}_{G}(b)
$$

Here $\nu_{b}$ is the Newton point of $b$ and $\operatorname{def}_{G}(b)$ is the defect of $b$. We refer to GHKR for the precise definitions. Such a dimension formula has been proved in equal characteristic by combining the works GHKR, V1, Ham2, but remains open in general in mixed characteristic. In fact, before our work, it is not clear how to define the dimension of $X_{\leq \mu}(b)$ in mixed characteristic in general, and this formula only makes sense for some special triples $(G, b, \mu)$ when (3.1.1) can be interpreted as the $\overline{\mathbb{F}}_{p}$-points of some moduli spaces of $p$-divisible groups (a.k.a. RZ spaces). In the case when the RZ spaces are of PEL type, this dimension formula was proved recently by Hamacher $([\mathrm{Ham} 1]$ ) and some special cases were proved by earlier work of Viehmann ( $\mathrm{V} 2, \mathrm{~V} 3]$ ).

Theorem 3.1. Rapoport's conjecture (3.1.3) holds in general.

Not surprisingly, the machinery developed so far in the paper allows us to imitate the the arguments in equal characteristic with only a few justifications. First, one can argue as in GHKR, Ham2 to reduce the general Rapoport's conjecture to the case when $b$ is superbasic. It was shown in GHKR, CKV that if $G$ is of adjoint type, superbasic $\sigma$-conjucay classes exist only when $G=\mathrm{PGL}_{n}$ or $G=\operatorname{Res}_{E / F} \mathrm{PGL}_{n}$, where $E / F$ is an unramified extension. The case when $G=\mathrm{PGL}_{n}$ was treated by Viehmann [V1] (in the equal characteristic situation but the same arguments apply here). We reduce the case $G=\operatorname{Res}_{E / F} \mathrm{PGL}_{n}$ to the case $G=\mathrm{PGL}_{n}$ and then apply [V1]. This in particular gives a shorter proof of the main result of Ham2] (but it uses [V1]). We sketch the arguments in what follows.

Remark 3.2. This is a side remark arising as a comment by G. Pappas. Although the algebro-geometric structure on $X_{\leq \mu}(b)$ was not known before, the authors of [CKV] defined a notation of the set of connected components $\pi_{0}\left(X_{\leq \mu}(b)\right)$ for $X_{\leq \mu}(b)$. It is clear that if two points on $X_{\leq \mu}(b)$ are in the same connected component in the sense of loc. cit., they are in the same connected component under the Zariski topology. If in their definition arbitrary test rings (rather than smooth rings) are allowed 13 , the converse holds. On the other hand, it seems that their arguments can be adapted to our setting to prove that the structure of connected components of $X_{\leq \mu}(b)$ in our sense is also given by the statement of [CKV] Theorem 1.1], and therefore it follows a posteriori that the two notions are the same. In any case, when $X_{\leq \mu}(b)$ is the set of $\mathbb{F}_{p}$-points of a Rapoport-Zink space, their $\pi_{0}$ coincides with the $\pi_{0}$ of the RZ space, and by Proposition 3.12 below, also coincides with $\pi_{0}$ of $X_{\leq \mu}(b)$ as the perfection of an algebraic space.

3.1.2. Now one can argue as in GHKR, Proposition 5.6.1, Theorem 5.8.1] to reduce the Rapoport conjecture for general $(G, \mu, b)$ to the case $b$ is basic. Namely the Newton point $\nu_{b}$ is defined over $F$, whose centralizer in $G$ is a rational Levi $M$. One can find a representative in the $\sigma$-conjugacy class of $b$ that is contained in $M(L)$. We rename this representative by $b$. So $b$ is basic in $M(L)$. Then the arguments reduce Rapoport's conjecture for $(G, b, \mu)$ to $\left(M, b, \mu_{M}\right)$ (for various $\mu_{M}$ ). These arguments relies on their Proposition 5.3.1 and 5.4.3. The proof of Proposition 5.3.1 in loc. cit. applies to the current setting. Note that the arguments involve an $M$-equivariant isomorphism $N \simeq \mathfrak{n}$. In the equal characteristic situation, this isomorphism makes sense either as $F$-schemes of as $k$-ind-schemes. In our

\footnotetext{
${ }^{13}$ In loc. cit., it was conjectured that these two definitions coincide.
} 
sitting, it only makes sense as an isomorphism of $F$-schemes. But it still makes sense to talk about the $p$-adic loop space of $\mathfrak{n}$ so the arguments in $\S 4$ of loc. cit. apply. The proof of Proposition 5.4.3 in loc. cit. extends verbatim in mixed characteristic, by taking account of the Lefschetz trace formula for separated pfp perfect algebraic spaces A.3.4 A special case of this type argument has been given in the proof of Proposition 2.9 (where $M=T$ ).

As explained in loc. cit., even $b$ is basic for $G$, it still might happen that $b$ is contained in a proper Levi subgroup of $G$. A basic $\sigma$-conjugacy class that does not meet in proper Levi subgroups of $G$ defined over $F$ is called a superbasic conjugacy class. Therefore, it is enough to prove Rapoport's conjecture in the case $b$ is superbasic. In addition, one can assume that $G=G^{\text {ad }}$ is simple of adjoint type. Recall that [GHKR, CKV] shows that the only possibility that a superbasic $b$ exists is when $G=\operatorname{Res}_{E / F} \mathrm{PGL}_{n}$ for some unramified extension $E / F$.

Remark 3.3. P. Hamacher Ham1 also observed that similar arguments of [GHKR hold in mixed characteristic. The difference between his approach and our approach is that he replaced the actual dimensions involved in the arguments by the numerical dimensions (defined by himself, which coincides the actual dimensions by the Lefschetz trace formula once the scheme structure on $N(F)$ is justified).

\subsubsection{It remains to prove that}

Proposition 3.4. Rapoport's conjecture holds in the case $G=\operatorname{Res}_{E / F} \mathrm{GL}_{n}$ and $b$ is superbasic.

Remark 3.5. This proposition has been proved by Hamacher when $F=\mathbb{Q}_{p}$ and $\mu$ is minsucle. Our method is different and is simpler, but it uses V1].

Proof. We first reduce the case $G=\operatorname{Res}_{E / F} \mathrm{GL}_{n}$ to the case $G=\mathrm{GL}_{n}$.

We start with a generalization of affine Deligne-Lusztig varieties. Let $H$ be a general unramified group over $E$. First observe that $X_{\leq \mu}(b)$ can be defined as the following Cartesian pullback

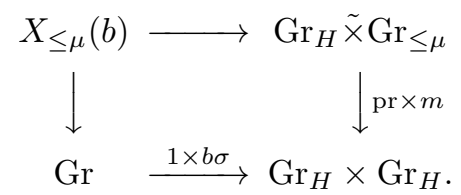

Now by replacing $\mu$ by a sequence of dominant coweights $\mu_{\bullet}$, we can form a convolution version of affine Deligne-Lusztig variety

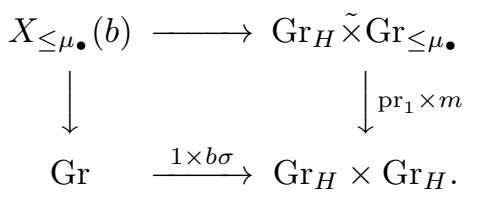

Concretely, $X_{\leq \mu \bullet}$ (b) classifies the following commutative diagram.

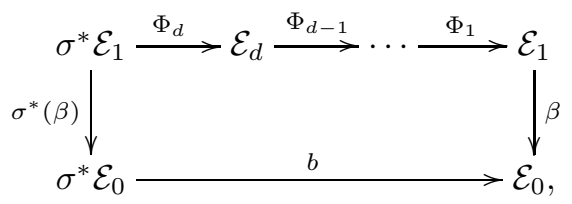

such that $\operatorname{Inv}_{x}\left(\Phi_{i}\right) \leq \mu_{i}$ for every $x \in \operatorname{Spec} R$. Note that in equal characteristic case, this is the local version of the moduli space of iterated Shtukas. $H$.

In the sequel, we write $X_{\leq \mu}^{H}(b)$ for $X_{\leq \mu}(b)$ if we want to emphasize the underlying group 
Now we start our reduction step. Let $\Sigma$ denote the set of embeddings $\tau: E \rightarrow L$ over $F$. Then $\operatorname{Gal}(L / F)$ acts transitively on $\Sigma$. We fix $\tau_{0} \in \Sigma$ and let $\tau_{i}=\sigma^{i}\left(\tau_{0}\right), i=0,1,2, \ldots, d-1$, where $d=[E: F]$.

Now assume that $G=\operatorname{Res}_{E / F} H$, for some unramified group $H$ over $E$. so $G \otimes L \simeq$ $\prod_{\tau \in \Sigma} H$. Let $\mu$ be a dominant coweight of $G_{L}$, so that under the above isomorphism, we obtain a sequence of dominant coweights $\mu_{\bullet}=\left(\mu_{\tau_{0}}, \ldots, \mu_{\tau_{d-1}}\right)$ of $H$. Similarly, $b \in G(L)$ gives $\left(b_{\tau}\right) \in \prod_{\tau \in \Sigma} H(L)$.

For an $\overline{\mathbb{F}}_{p}$-algebra $R$, we identify $(\mathcal{E}, \beta) \in \operatorname{Gr}_{G}$ with $\left(\mathcal{E}_{\tau}, \beta_{\tau}\right) \in \prod_{\tau \in \Sigma} \operatorname{Gr}_{H}$ in an obvious way. Then the condition (3.1.2) is equivalent to the commutativity of the following diagram

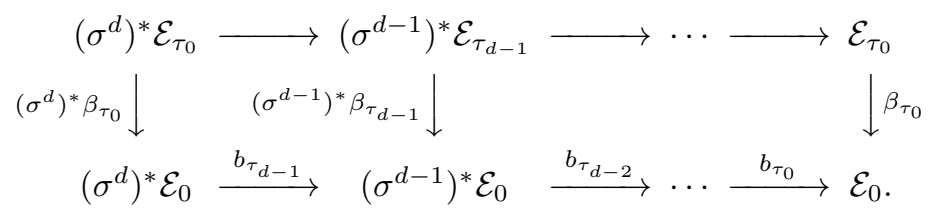

If we denote by $\mathrm{Nm} b=b_{\tau_{0}} \cdots b_{\tau_{d-1}}$, we see that

Lemma 3.6. If $G=\operatorname{Res}_{E / F} H$, then $X_{\leq \mu}^{G}(b) \simeq X_{\leq \mu \bullet}^{H}(\operatorname{Nm} b)$.

Remark 3.7. The map $b \mapsto \mathrm{Nm} b$ defines a map from the $\sigma$-conjugacy class of $G(L)$ to the $\sigma^{d}$-conjugacy class $H(L)$.

Now, assume that the dimension formula for affine Deligne-Lusztig varieties for $H$ has been established, we calculate the dimension of $X_{\leq \mu}^{G}(b)=X_{\leq \mu}^{H}(\mathrm{Nm} b)$. Recall the convolution Grassmannian (for $H$ )

$$
m: \mathrm{Gr}_{\leq \mu \bullet} \rightarrow \mathrm{Gr}_{\leq\left|\mu_{\bullet}\right|} .
$$

By (3.1.4) and (3.1.5), the following diagram is Cartensian

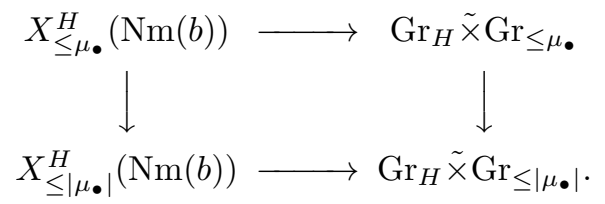

By (the proof of) Proposition 2.3, for $\lambda$, the fiber $m^{-1}\left(\varpi^{\lambda}\right)$ is of dimension $\leq\left(\rho_{H},\left|\mu_{\bullet}\right|-\lambda\right)$. From this, we see that

$$
\begin{aligned}
\operatorname{dim} X_{\leq \mu \bullet}^{H}(\operatorname{Nm}(b)) & =\max _{\lambda} \operatorname{dim} X_{\leq \lambda}^{H}(\operatorname{Nm} b)+\left(\rho_{H},\left|\mu_{\bullet}\right|-\lambda\right) \\
& =\left(\rho_{H},\left|\mu_{\bullet}\right|-\nu_{\mathrm{Nm} b}\right)-\frac{1}{2} \operatorname{def}_{H}(\operatorname{Nm} b) \\
& =\left(\rho_{G}, \mu-\nu_{b}\right)-\frac{1}{2} \operatorname{def}_{G}(b) .
\end{aligned}
$$

Therefore, it remains to prove the case for $G=\mathrm{GL}_{n}$ and $b$ superbasic. Now one can argue exactly the same as [V1] to complete the proof.

3.2. Affine Deligne-Lusztig varieties and Rapoport-Zink spaces. Let us recall RapoportZink (RZ) spaces. In the PEL case, they are defined by Rapoport-Zink in their original work [RZ]. In a more general situation but under the assumption that the group is unramfied, they are recently defined by $\mathrm{Kim}\left[\mathrm{Kim}\right.$. We assume (for simplicity) that $k=\overline{\mathbb{F}}_{p}$ is algebraically closed. To follow the standard notation, we write $W=W(k)$ (which was usually denoted by $\mathcal{O}$ before). Let $L=W \otimes \mathbb{Q}_{p}$. We use $F$ to denote $\sigma$-linear map of $L$-linear vector spaces (unlike the rest part of the paper where $F$ denotes a local field). Let Nilp ${ }_{W}$ denote the category of $W$-algebras on which $p$ acts nilpotently.

We will start with a reductive group $G$ over $\mathbb{Z}_{p}$, a geometric conjugacy class of cocharacters $\mu: \mathbb{G}_{m} \rightarrow G$, and a $\sigma$-conjugacy class $b$ of $G(L)$ with a representative in $G(W) p^{\mu} G(W)$, still denoted by $b$. We assume that there exists a free $\mathbb{Z}_{p}$-lattice $\Lambda^{14}$ and a faithful presentation

$$
\rho: G \rightarrow \mathrm{GL}(\Lambda)
$$

\footnotetext{
${ }^{14}$ Our $\Lambda$ corresponds to $\Lambda^{*}$, and $\mu$ corresponds to $-\mu$ in $\underline{\text { Kim }}$.
} 
such that the cocharacter $\rho \mu: \mathbb{G}_{m} \rightarrow \mathrm{GL}(\Lambda \otimes W)$ has weights 0,1 . We fix a representative $\mu$. Let

$$
\Lambda \otimes W=\Lambda^{0} \oplus \Lambda^{1}
$$

be the decomposition of $\Lambda \otimes W$ according to the weights of $\mu$, which in turn induces a filtration $\operatorname{Fil}^{0} \Lambda=\Lambda \supset \operatorname{Fil}^{1} \Lambda=\Lambda^{1}$. We assume that $\operatorname{rk} \Lambda^{1}=n$, and $\operatorname{rk} \Lambda=h$. This is equivalent to say that $\rho \mu: \mathbb{G}_{m} \rightarrow \mathrm{GL}(\Lambda)$ is the $n$ the fundamental coweight of $\operatorname{GL}(\Lambda)$.

Let $\Lambda^{\otimes}$ denote the tensor algebra of $\Lambda \oplus \Lambda^{*}$. Note that $\Lambda^{\otimes}=\left(\Lambda^{*}\right)^{\otimes}$, and elements in $\Lambda^{\otimes}$ are called tensors. We choose a finite collection of tensors $\left\{s_{i} \in \Lambda^{\otimes}, i \in I\right\}$ such that $\rho: G \subset \mathrm{GL}(\Lambda)$ is the schematic stabilizer of this collection. I.e. $G=\operatorname{Aut}\left(\Lambda,\left\{s_{i}, i \in I\right\}\right)$. For example, if $G=\mathrm{GL}_{h}$, we can let $\left\{s_{i}\right\}$ be the empty set. Note that $\operatorname{Aut}\left(\Lambda,\left\{s_{i}, i \in\right.\right.$ $\left.I\}, \operatorname{Fil}^{*}(\Lambda \otimes W)\right)$ is a parabolic subgroup of $G_{W}$, determined by $\mu$, denoted by $P_{\mu}$.

Note that by our assumption and the classical Dieudonné theory, there exists a $p$-divisible group $\mathbb{X}_{0}$ of dimension $n$ and height $h$, over $\overline{\mathbb{F}}_{p}$, together an isomorphism

$$
\varepsilon: \mathbb{D}\left(\mathbb{X}_{0}\right) \simeq \Lambda \otimes_{\mathbb{Z}_{p}} W
$$

where $\mathbb{D}\left(\mathbb{X}_{0}\right)$ is the contravariant Dieudonné module of $\mathbb{X}_{0}$, equipped with $(F, V)$, such that:

(1) $\varepsilon F=\rho(b)\left(\operatorname{id}_{\Lambda} \otimes \sigma\right) \varepsilon$;

(2) $\varepsilon\left(\operatorname{Lie} \mathbb{X}_{0}\right)^{*}=\operatorname{Fil}^{1} \Lambda \otimes \overline{\mathbb{F}}_{p}$.

The pair $\left(\mathbb{X}_{0}, \varepsilon\right)$ is unique up to a unique isomorphism and we fix it in the sequel.

Note that via $\varepsilon$, we can regard $s_{i}$ as tensors in $\mathbb{D}\left(\mathbb{X}_{0}\right)^{\otimes}$. Since $G$ fixes $\left\{s_{i}\right\}, b \sigma$ fixes $\left\{s_{i}\right\}$. So $\left\{s_{i}\right\}$ are $F$-invariant. In addition, the cocharacter $\rho \mu: \mathbb{G}_{m} \rightarrow \operatorname{GL}(\Lambda)$ also fixes $s_{i}$. Therefore, $s_{i}$ are in the $\mathrm{Fil}^{0} \Lambda^{\otimes}$. Let us denote the Dieudonné module of the constant p-divisible group $\mathbb{Q}_{p} / \mathbb{Z}_{p}$ by $1:=\mathbb{D}\left(\mathbb{Q}_{p} / \mathbb{Z}_{p}\right)=W$, with the $\sigma$-linear map $F: W \rightarrow W$ sending $F(1)=1$ and the decreasing filtration $F^{0} W=W, F^{1} W=\left(\operatorname{Lie} \mathbb{Q}_{p} / \mathbb{Z}_{p}\right)^{*}=0$. Then each $s_{i}$ can be regarded as a map $1 \rightarrow \mathbb{D}\left(\mathbb{X}_{0}\right)^{\otimes}$, compatible with the filtrations, and such that the induced map $1 \rightarrow \mathbb{D}\left(\mathbb{X}_{0}\right)\left[\frac{1}{p}\right]$ is Frobenius-invariant. Following $[\mathrm{Kim}$, we call such a map a (crystalline-) Tate tensor of $\mathbb{X}_{0}$. It admits the following generalization to a family of $p$-divisible groups.

Let $R \in \operatorname{Nilp}_{W}$ and a $p$-divisible group $\mathbb{X}$ on $\operatorname{Spec} R$, let $\mathbb{D}(\mathbb{X})$ denote its contravariant Dieudonné crystal. This is an $F$-crystals on $\operatorname{Spec} R$, by which we mean a locally free crystal $\mathbb{D}(\mathbb{X})$ on $\operatorname{CRIS}(R / W)$, with a $\sigma$-linear map (the Frobenius map) $F: \mathbb{D}(\mathbb{X}) \rightarrow \mathbb{D}(\mathbb{X})$, which induces a bijection after inverting $p$. In addition, there is a decreasing filtraion Fil $\bullet \mathbb{D}(\mathbb{X})_{R}$ on $\mathbb{D}(\mathbb{X})_{R}$ (the value of $\mathbb{D}(\mathbb{X})$ at the trivial PD-thickening $R \stackrel{\text { id }}{\rightarrow} R$ ) whose associated graded is locally free over $R$. Namely, Fil ${ }^{0} \mathbb{D}(\mathbb{X})_{R}=\mathbb{D}(\mathbb{X})_{R}$, Fil $^{1} \mathbb{D}(\mathbb{X})_{R}=\left({\text { Lie } \mathbb{X})^{*} \text { and Fil }}^{2} \mathbb{D}(\mathbb{X})_{R}=\right.$ 0 . Note that $\mathbb{D}(\mathbb{X})^{\otimes}$ is also an $F$-crystal with a filtration Fil $\mathbb{D}(\mathbb{X})_{R}^{\otimes}$. For example, let $1=\mathbb{D}\left(\mathbb{Q}_{p} / \mathbb{Z}_{p}\right)$ be the filtered $F$-crystal given by the Dieudonné module of the constant $p$ divisible group $\mathbb{Q}_{p} / \mathbb{Z}_{p}$. Then $1_{R^{\prime}}=R^{\prime}$ for every PD-thickening $R^{\prime} \rightarrow R$ and $F: 1_{R^{\prime}} \rightarrow 1_{R^{\prime}}$ sending $F(1)=1$. In addition, Fil $^{1} 1_{R}=0$. Then we call a (crystalline-)Tate tensor of $\mathbb{X}$ a morphism $t: 1 \rightarrow \mathbb{D}(\mathbb{X})^{\otimes}$ of crystals, such that $t_{R}: 1_{R} \rightarrow \mathbb{D}(\mathbb{X})_{R}^{\otimes}$ is compatible with the filtrations, and such that the induced map $t: 1 \rightarrow \mathbb{D}(\mathbb{X})^{\otimes}\left[\frac{1}{p}\right]$ is Frobenius-invariant.

For a $p$-divisible group over a general base $R$, the notion of Tate tensors may not be well-behaved. Following $\underline{\mathrm{Kim}}$, let Nilp $\mathrm{sm}_{W}$ denote the full subcategory of $\mathrm{Nilp}_{W}$ consisting of formally smooth formally finitely generated $W / p^{m}$-algebras for $m>0$. We regard $R \in$ Nilp ${ }_{W}^{\mathrm{sm}}$ as affine formal scheme $\operatorname{Spf} R$.

Definition 3.8. The RZ space associated to $(G, b, \mu)$ is the functor $\breve{\mathcal{M}}(G, b, \mu)$ on $\operatorname{Nilp}_{W}^{\mathrm{sm}}$ classifying: for every $R \in \mathrm{Nilp}_{W}^{\mathrm{sm}}$, regarded as an affine formal scheme,

(1) a $p$-divisible group $\mathbb{X}$ on $\operatorname{Spec} R$;

(2) a collection of cyrstalline-Tate tensors $\left\{t_{i}\right\}, i \in I$ of $\mathbb{X}$;

(3) a quasi-isogeny $\iota: \mathbb{X}_{0} \otimes_{k} R / J \rightarrow \mathbb{X} \otimes_{R} R / J$ that sends $t_{i}$ to $s_{i} \otimes 1$ for $i \in I$, where $J$ is some (and therefore any) ideal of definition of $R$. 
such that

(*) the $R$-scheme

$$
\operatorname{Isom}\left(\left(\mathbb{D}(\mathbb{X})_{R},\left\{t_{i}\right\}, \operatorname{Fil} \bullet\left(\mathbb{D}(\mathbb{X})_{R}\right)\right),\left(\Lambda \otimes_{\mathbb{Z}_{p}} R,\left\{s_{i} \otimes 1\right\}, \text { Fil } \Lambda^{\bullet} \otimes_{\mathbb{Z}_{p}} R\right)\right)
$$

that classifies the isomorphisms between locally free sheaves $\mathbb{D}(\mathbb{X})_{R}$ and $\Lambda \otimes_{\mathbb{Z}_{p}} R$ on Spec $R$ preserving the tensors and the filtrations is a $\left(P_{\mu} \otimes_{W} R\right)$-torson;

Remark 3.9. (i)Our definition is slightly different from the original definition given in Kim. But it is not hard to see the condition $\left(^{*}\right)$ combines the Item (2) and (3) in [Kim, Definition 3.6].

(ii) As explained in $\overline{\mathrm{Kim}}, \breve{\mathcal{M}}(G, b, \mu)$ is independent of the choice of $\rho: G \rightarrow \operatorname{GL}(\Lambda)$ up to isomorphism.

The main theorem of Kim $\underline{\operatorname{Kim}}$ asserts

Theorem 3.10. Assume that either $p>2$. Then $\breve{\mathcal{M}}(G, b, \mu)$ is represented by a separated formal scheme, formally smooth and locally formally of finite type over $W$.

Remark 3.11. The definition of general $\breve{\mathcal{M}}(G, b, \mu)$ is technical. E.g. it is not clear how to give the description of Spec $R$-points of $\mathcal{M}(G, b, \mu)$ for general $(G, b, \mu)$ and general $R \in$ Nilp $_{W}$. But this does not bother us. For our purpose (the following Proposition 3.12), it is enough to know:

(1) $\breve{\mathcal{M}}(G, b, \mu)$ is representable;

(2) If $(G, b, \mu)=\left(\mathrm{GL}_{h}, b, \omega_{n}\right)$, one can choose $\left\{s_{i}\right\}$ to be the empty set. In this case, the moduli problem $\breve{\mathcal{M}}_{n, h}:=\breve{\mathcal{M}}(G, b, \mu)$ can be extended to the whole category $\operatorname{Nilp}_{W}$, as in [RZ]: for every $R \in \operatorname{Nilp}_{W}, \breve{\mathcal{M}}_{n, h}(R)$ classify the groupoid of pairs $(\mathbb{X}, \iota)$, where $\mathbb{X}$ is a $p$-divisible group over $\operatorname{Spec} R$, and $\iota: \mathbb{X}_{0} \otimes_{k} R / p \rightarrow \mathbb{X} \otimes_{R} R / p$ is a quasi-isogeny. Note that the Condition $\left(^{*}\right)$ automatically holds. Rapoport-Zink proved that $\mathcal{M}_{n, h}$ is represented by a separated formal scheme, formally smooth and formally locally of finite type over $W$.

(3) For general $(G, b, \mu)$, a representation $\rho: G \rightarrow \mathrm{GL}(\Lambda)$ as above induces a closed embedding of $\breve{\mathcal{M}}(G, b, \mu)$ into $\mathcal{M}_{n, h}$.

(4) There is a canonical bijection $\breve{\mathcal{M}}(G, b, \mu)(k) \simeq X_{\mu}(b)(k)$ compatible with the embed$\operatorname{dings} \breve{\mathcal{M}}(G, b, \mu) \rightarrow \mathcal{M}_{n, h}$ and $X_{\mu}(b) \rightarrow X_{\rho \mu}(\rho(b))$ (see [Kim, Proposition 3.8.4]).

We write $\overline{\mathcal{M}}_{\mu}(b)$ for the special fiber of $\breve{\mathcal{M}}(G, b, \mu)$. As a corollary, we have

Proposition 3.12. Fixing $\left(\mathbb{X}_{0}, \varepsilon\right)$ as bove. There is a canonical isomorphism $X_{\mu}(b) \simeq$ $\overline{\mathcal{M}}_{\mu}(b)^{p^{-\infty}}$. In particular, $\operatorname{dim} \overline{\mathcal{M}}_{\mu}(b)_{\mathrm{red}}=\operatorname{dim} X_{\mu}(b)$.

Note that this proposition in particular implies describes the value of $\breve{\mathcal{M}}(G, b, \mu)$ on a perfect ring $R$ (which is not obvious from Definition 3.8).

Proof. We first prove this for $(G, b, \mu)=\left(\mathrm{GL}_{h}, b, \omega_{n}\right)$, using the fact that the moduli problem is defined on $\mathrm{Nilp}_{W}$. The key input is a theorem of Gabber (see also [La, §6]) on $p$-divisible groups over perfect rings. We write $\overline{\mathcal{M}}^{p^{-\infty}}$ instead of $\overline{\mathcal{M}}_{\mu}(b)^{p^{-\infty}}$ for simplicity.

We first construct $X_{\mu}(b) \rightarrow \overline{\mathcal{M}}^{p^{-\infty}}$ as follows. Let $R$ be a perfect $\overline{\mathbb{F}}_{p}$-algebra. We write $\sigma: R \rightarrow R$ for the Forbenius endomorphism. Let $(\mathcal{E}, \beta) \in X_{\mu}(b)(R)$. We obtain a crystal $\mathbb{D}:=\mathcal{E} \times^{G, \rho} \Lambda$ over $R$ (= a locally free sheaf on $W(R)$ ), with $F=\beta^{-1}(b \sigma) \beta$. I.e., the

\footnotetext{
${ }^{15}$ Recall that $P_{\mu}$ is the automorphism group scheme of $\left(\Lambda,\left\{s_{i}\right\}\right.$, Fil $\left.{ }^{\bullet} \Lambda\right)$.
} 
following diagram is commutative

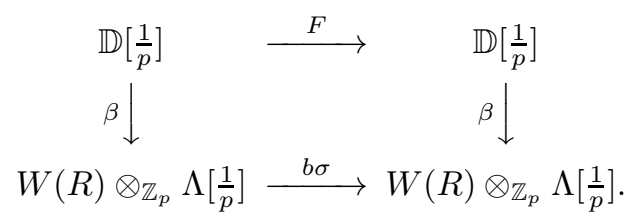

Note that $\omega_{n}: \mathbb{G}_{m} \rightarrow \mathrm{GL}(\Lambda)$ is the $n$th fundamental coweight of $\operatorname{GL}(\Lambda)$, and therefore the condition as in (3.1.2) together with Lemma 1.6 implies that $F: \sigma^{*} \mathbb{D} \rightarrow \mathbb{D}$ is regular and the quotient is a locally free module of rank $n$ on $W(R) / p=R$. Applying Lemma 1.6] again to the quasi-isogeny (of crystals) $V=p F^{-1}: \mathbb{D} \rightarrow \sigma^{*} \mathbb{D}$, we see that $V$ is regular. Therefore, there is a $\sigma^{-1}$-linear map $V: \mathbb{D} \rightarrow \mathbb{D}$ such that $F V=V F=p$. By Gabber's theorem (see also $[\mathrm{La}, \S 6]$ ), there is a $p$-divisible group $\mathbb{X}$ on $\operatorname{Spec} R$ together with a quasi-isogeny $\iota: \mathbb{X} \rightarrow\left(\mathbb{X}_{0}\right)_{R}$ such that $\mathbb{D}=\mathbb{D}(\mathbb{X})$ and the induces map $\mathbb{D}(\iota): \mathbb{D}\left[\frac{1}{p}\right] \rightarrow W(R)\left[\frac{1}{p}\right] \otimes \Lambda$ is the same as $\beta$.

Conversely, we construct $\overline{\mathcal{M}}^{p^{-\infty}} \rightarrow X_{\mu}(b)$ as follows. Let $R$ be a perfect $\overline{\mathbb{F}}_{p}$-algebra. Let $(\mathbb{X}, \iota)$ be an object in $\overline{\mathcal{M}}(R)$. Then we have the $\mathrm{GL}(\Lambda)$-torsor

$$
\mathcal{E}=\operatorname{Isom}\left(\left(\mathbb{D}(\mathbb{X}),\left\{t_{i}, i \in I\right\}\right),\left(\Lambda \otimes_{\mathbb{Z}_{p}} W(R),\left\{s_{i} \otimes 1\right\}\right)\right) .
$$

The quasi-isogeny $\iota$ defines an isomorphism

$$
\mathbb{D}(\mathbb{X})\left[\frac{1}{p}\right] \simeq \Lambda \otimes_{\mathbb{Z}_{p}} W(R)\left[\frac{1}{p}\right],
$$

and therefore defines an isomorphism $\beta: \mathcal{E}\left[\frac{1}{p}\right] \simeq \mathcal{E}_{0}\left[\frac{1}{p}\right]$. The map $(\mathbb{X}, \iota) \mapsto(\mathcal{E}, \beta)$ defines a map $\overline{\mathcal{M}}^{p^{-\infty}} \rightarrow \operatorname{Gr}_{\mathrm{GL}_{h}}$. It is clear that $\operatorname{Inv}_{x}(F) \leq \omega_{n}$ for every $x \in \operatorname{Spec} R$, therefore $(\mathcal{E}, \beta) \in X_{\mu}(b)$. We thus defines a map $\overline{\mathcal{M}}^{p^{-\infty}} \rightarrow X_{\mu}(b)$.

Now we consider the general $(G, b, \mu)$. The representation $\rho: G \rightarrow \operatorname{GL}(\Lambda)$ on the one hand gives an embedding $\breve{\mathcal{M}}(G, b, \mu) \rightarrow \breve{\mathcal{M}}_{n, h}$ as proved in $\overline{\mathrm{Kim}}$, and on the other hand gives an embedding $X_{\mu}(b) \rightarrow X_{\omega_{n}}(\rho(b))$. To identify $\overline{\mathcal{M}}_{G}^{p^{-\infty}}$ with $X_{\mu}(b)$, it is then enough to check at the level of points. But this has been done by [Kim, Proposition 3.8.4].

Corollary 3.13. Rapoport's conjecture of the dimension formula holds for the reduced schemes of the Rapoport-Zink spaces.

As mentioned before, in the PEL case, this has also been shown by Hamacher Ham1.

\section{Appendix A. Generalities on perfect schemes}

This section can be regarded as a user's guide to perfect schemes and perfect algebraic spaces, which is the setting we will work with in the paper. We include some discussions more general than needed in the paper. The main result is Proposition A.28, which explains the construction of the quotients in this setting.

\section{A.1. Perfect schemes and perfect algebraic spaces.}

A.1.1. Let $k$ be a field, and let $\mathrm{Aff}_{k}$ denote the category of affine $k$-schemes, i.e. the category opposite to the category $k$-alg of $k$-algebras. We endow $\mathrm{Aff}_{k}$ with the fpqc topology. I.e., a cover of $R$ is a faithfully flat map $R \rightarrow R^{\prime}$. Recall in [BL, a $k$-space is a sheaf on $\mathrm{Aff}_{k}$ with respect to this topology. Explicitly, a space $\mathcal{F}$ is a covariant functor $k$-alg $\rightarrow$ Set that respects finite products, and such that if $R \rightarrow R^{\prime}$ is faithfully flat,

$$
\mathcal{F}(R) \rightarrow \mathcal{F}\left(R^{\prime}\right) \rightrightarrows \mathcal{F}\left(R^{\prime} \otimes_{R} R^{\prime}\right)
$$

is an equalizer. The morphisms between two spaces are natural transforms of functors. The category of $k$-spaces is denoted by $\mathrm{Sp}_{k}$. It is known after Grothendieck that it contains the 
category $\operatorname{Sch}_{k}$ of $k$-schemes as a full subcategory. Recall that a map $f: \mathcal{F} \rightarrow \mathcal{G}$ in $\operatorname{Sp}_{k}$ is called schematic if for every scheme $T$, the fiber product $\mathcal{F} \times \times_{\mathcal{G}} T$ is a scheme.

In this paper, we need to consider a larger subcategory in $\mathrm{Sp}_{k}$. Recall that an algebraic space is an étale sheaf $X$ on Aff ${ }_{k}$ such that: (i) $X \rightarrow X \times X$ is schematic ; (ii) There exists an étale surjective map $U \rightarrow X$, where $U$ is a scheme.

By an fpqc algebraic space, we mean an algebraic space that is an fpqc sheart 16 . We denote by $\mathrm{AlgSp}_{k}$ the category of fpqc algebraic spaces. We have full embeddings $\operatorname{Sch}_{k} \subset \operatorname{AlgSp}_{k} \subset$ $\mathrm{Sp}_{k}$.

Remark A.1. In literature as $\mathrm{Kn}, \mathrm{LMB}$, it sometimes requires that $X$ is quasi-separated, i.e. the diagonal $X \rightarrow X \times X$ is quasi-compact. It is shown in [LMB Theorem(A.4)] and [St, Tag03W8] that in the presence of quasi-separatedness assumption (which will always be the case in the examples we consider in the paper), algebraic spaces are fpqc sheaves, so belong to $\mathrm{AlgSp}_{k}$.

A map $f: \mathcal{F} \rightarrow \mathcal{G}$ of $k$-spaces is called representable if for every affine scheme $T, \mathcal{F} \times \mathcal{G} T$ is represented by an fpqc algebraic space. It is call fpqc if in addition $\mathcal{F} \times \mathcal{G} T$ is faithfully flat over $T$, and there is a quasi-compact open subset $U$ of $\mathcal{F} \times_{\mathcal{G}} T$ that maps surjectively to $T$. Recall that fpqc maps are effective epimorphisms in $\mathrm{Sp}_{k}$. I.e. If $U \rightarrow X$ is an fpqc map of spaces, then for every space $\mathcal{F}$, the following diagram is an equalizer

$$
\operatorname{Hom}_{\mathrm{Sp}_{k}}(X, \mathcal{F}) \rightarrow \operatorname{Hom}_{\mathrm{Sp}_{k}}(U, \mathcal{F}) \rightrightarrows \operatorname{Hom}_{\mathrm{Sp}_{k}}\left(U \times_{X} U, \mathcal{F}\right)
$$

In particular, any fpqc sheaf on Aff ${ }_{k}$ extends uniquely to an fpqc sheaf on $\operatorname{Sch}_{k}$ (although we do not use the latter in this paper).

A.1.2. Let $k$ be a perfect field of characteristic $p>0$. Let $R$ be a $k$-algebra. Let $\sigma: R \rightarrow R$ denote the Frobenius map $\sigma(a)=a^{p}$. A ring is called perfect if $\sigma$ is an isomorphism. For any $R$, we can form the perfection (or sometimes called the perfect closure) of $R$ as $R^{p^{-\infty}}=\lim _{\sigma} R$. The functor $R \mapsto R^{p^{-\infty}}$ is left adjoint to the forgetful functor from the category of perfect $k$-algebras to the category of all $k$-algebras.

Similarly, a $k$-scheme (resp. algebraic space) $X$ is called perfect if its Frobenius endomorphism $\sigma_{X}: X \rightarrow X$ is an isomorphism. We write $\sigma$ for $\sigma_{X}$ if no confusion will likely arise. The category of perfect schemes (resp. perfect fpqc algebraic spaces) over $k$ is denoted by $\operatorname{Sch}_{k}^{\mathrm{pf}}\left(\right.$ resp. $\left.\operatorname{AlgSp}{ }_{k}^{\mathrm{pf}}\right)$.

Recall that $X \rightarrow X^{p^{-\infty}}=\lim _{\sigma} X$ is a right adjoint functor of the embedding $\operatorname{Sch}_{k}^{\mathrm{pf}} \subset \operatorname{Sch}_{k}$. In other words, for a scheme $X$, there is a tautological map $\varepsilon: X^{p^{-\infty}} \rightarrow X$ such that for any perfect $k$-scheme $Y$,

$$
\operatorname{Hom}\left(Y, X^{p^{-\infty}}\right)=\operatorname{Hom}(Y, X) \text {. }
$$

This way, we can regard $\operatorname{Sch}_{k}^{\mathrm{pf}} \simeq \operatorname{Sch}_{k}\left[\sigma^{-1}\right]$, the localization of $\operatorname{Sch}_{k}$ with respect to $\sigma$.

The same story holds for algebraic spaces, by noting that Frobenius endomorphisms commute not only with Zariski localizations, but also with étale localizations. More precisely, we have

Lemma A.2. For any étale morphism of algebraic spaces $X \rightarrow Y$, the map $X \rightarrow X \times_{Y, \sigma_{Y}} Y$ is an isomorphism.

Proof. We first assume that $X$ is a scheme. Then $X \rightarrow X \times_{Y, \sigma_{Y}} Y$ a schematic radical étale surjective map, and therefore is an isomorphism by [EGAIV, Theorem 17.9.1]. In general, choose an étale cover $U \rightarrow X$. Then we have $U \rightarrow U \times_{X, \sigma_{X}} X \rightarrow U \times_{Y, \sigma_{Y}} Y$, with the first and the composition maps being isomorphisms. Therefore, the second map is an isomorphism. But note that $U \times_{X, \sigma_{X}} X \rightarrow U \times_{Y, \sigma_{Y}} Y$ is just the base change of

\footnotetext{
${ }^{16}$ The author was informed by de Jong, who attributed to Gabber, that every algebraic space is an fpqc sheaf. However, it seems that no written proof is available at the moment.
} 
$X \rightarrow X \times_{Y, \sigma_{Y}} Y$ along the étale cover $U \times_{Y, \sigma_{Y}} Y \rightarrow Y$. Therefore, $X \rightarrow X \times_{Y, \sigma_{Y}} Y$ is also an isomorphism.

Corollary A.3. The embedding from the category of perfect algebraic spaces to the whole category of algebraic spaces admits a right adjoint functor, $X \rightarrow X^{p^{-\infty}}=\lim _{\sigma} X$.

Proof. Applying Lemma A.2 to the étale cover $U \rightarrow X$, we see that $\sigma: X \rightarrow X$ is an affine morphism. Then the diagonal of $X^{p^{-\infty}}=\lim _{\sigma} X$ is representable, and $U^{p^{-\infty}} \simeq$ $U \times_{X} X^{p^{-\infty}} \rightarrow X^{p^{-\infty}}$ is an étale cover. It is clear then that A.1.3) remains valid in this situation.

We call $X^{p^{-\infty}}$ the perfection of $X$. Note that if $X$ is an fpqc algebraic space, so is $X^{p^{-\infty}}$. So $X \mapsto X^{p^{-\infty}}$ restricts to a right adjoint of the embedding $\operatorname{AlgSp}_{k}^{\mathrm{pf}} \rightarrow \operatorname{AlgSp}_{k}$. In addition, by Lemma A.2, $\sigma_{X}$ is a universal homeomorphism. So $X^{p^{-\infty}} \rightarrow X$ is a universal homeomorphism.

The following statement is crucial for later applications. For an algebraic space $X$, we denote by $X_{e t}$ its small étale site with objects being algebraic spaces étale over $X$.

Proposition A.4. Let $X$ be an algebraic space over $k$ and let $X^{p^{-\infty}}$ denote its perfection. There the functor $(U \rightarrow X) \mapsto\left(U^{p^{-\infty}} \simeq U \times_{X} X^{p^{-\infty}} \rightarrow X^{p^{-\infty}}\right)$ induces an equivalence of étale sites $X_{e t} \simeq X_{e t}^{p^{-\infty}}$, and therefore the étale topos $\varepsilon^{*}: \widetilde{X}_{e t} \simeq \widetilde{X}_{e t}^{p^{-\infty}}: \varepsilon_{*}$.

Proof. First, assume that $X$ is a scheme. Then $X^{p^{-\infty}} \rightarrow X$ is an integral, radical surjection. Therefore by [SGA4, VIII 1.1], $(U \rightarrow X) \mapsto\left(U^{p^{-\infty}} \simeq U \times_{X} X^{p^{-\infty}} \rightarrow X^{p^{-\infty}}\right)$ induces an equivalence of subcategories of scheme objects in $X_{e t}$ and $X_{e t}^{p^{-\infty}}$. Then an argument similar to [CLO, Proposition A.1.3] shows that it induces a full equivalence $X_{e t} \simeq X_{e t}^{p^{-\infty}}$. Again, by a similar argument as [CLO, Proposition.A.1.3], the case when $X$ is an algebraic space also follows.

Remark A.5. In fact, the equivalence preserves the subcategories of scheme objects in $X_{e t}$ and $X_{e t}^{p^{-\infty}}$. Indeed, if $U$ is an algebraic space, and $U^{p^{-\infty}}$ is a scheme, then $U$ is a scheme. See Lemma A.6.

We list a few properties of morphisms that are preserved after passing to the perfection.

Lemma A.6. Let $f: X \rightarrow Y$ be a morphism of algebraic spaces over $k$, and let $f^{p^{-\infty}}$ : $X^{p^{-\infty}} \rightarrow Y^{p^{-\infty}}$ denote the corresponding perfection. The following properties hold for $f$ if and only if the same hold for $f^{p^{-\infty}}$ : (1) quasi-compact, (2) quasi-separated, (3) separated, (4) affine. In addition, if $f$ is either: (5) étale, (6) (faitfully) flat, (7) fpqc, so is $f^{p^{-\infty}}$.

Proof. (1)-(2) are clear. To prove (3), note that since $X^{p^{-\infty}} \rightarrow X^{p^{-\infty}} \times_{Y^{p^{-}}} X^{p^{-\infty}}$ is a closed embedding, it is universally closed, so $\Delta_{X / Y}: X \rightarrow X \times_{Y} X$ is universally closed. But $\Delta_{X / Y}$ is always a separated, locally of finite type monomorphism, and therefore is a closed embedding. The inverse direction is clear.

(4) By Lemma A.2 we can assume that $Y$ is an affine scheme. Then if $X$ is affine so is $X^{p^{-\infty}}$. Conversely, if $X^{p^{-\infty}}$ is affine, then it is quasi-compact and separated, and so is $X$. As $X^{p^{-\infty}}=\varliminf_{\sigma} X, X$ is affine by [St, Tag07SE, Lemma 5.8].

(5) follows from Lemma A.2. To prove (6), we may assume that $f: X \rightarrow Y$ are affine schemes, so given by ring homomorphism $f: R \rightarrow R^{\prime}$. In addition, we may assume that $R$ is perfect. As $\left(R^{\prime}\right)^{p^{-\infty}}=\lim _{\sigma} R^{\prime}$, it is enough to show that the composition $R \rightarrow R^{\prime} \stackrel{\sigma^{n}}{\rightarrow} R^{\prime}$ is flat. But this map is the same as $R \stackrel{\sigma^{n}}{\simeq} R \rightarrow R^{\prime}$ and therefore is flat. Finally, (7) follows from (6). 
We will also consider the perfection of certain pro-algebraic spaces. Let $\left\{X_{i}\right\}$ be a projective limit of algebraic spaces, with the transition maps $X_{i+1} \rightarrow X_{i}$ given by affine morphisms. Then the pro-algebraic space $X=\lim _{i} X_{i}$ is also an algebraic space and it is easy to show

$$
X^{p^{-\infty}} \simeq \varliminf_{\swarrow} X_{i}^{p^{-\infty}}
$$

i.e. "perfections commute with inverse limits".

A.1.3. Let $H$ be an affine group scheme over $k$. It gives rise to a group object in $\operatorname{Sp}_{k}$. An $H$-torsor over a space $X$ is a space $E$ with a free $H$-action and an $H$-equivariant fpqc map $\pi: E \rightarrow X$ (where $X$ is endowed with the trivial $H$-action), such that the natural map $E \times H \rightarrow E \times{ }_{X} E$ is an isomorphism. If $X$ is an fpqc algebraic space $k$, then $E$ is represented by an fpqc algebraic space, affine over $X$. In addition,

Lemma A.7. If $X$ and $H$ are perfect, then $E$ is perfect.

Proof. Note that $E^{p^{-\infty}} \rightarrow X^{p^{-\infty}}$ is also an fpqc map by Lemma A.6, and therefore is an $H^{p^{-\infty}}$-torsor over $X^{p^{-\infty}}$. In addition, $E^{p^{-\infty}} \rightarrow E \times_{X, \varepsilon} X^{p^{-\infty}}$ is a morphism of $H^{p^{-\infty}}$ torsors, where $H^{p^{-\infty}}$ acts on $E$ via $H^{p^{-\infty}} \rightarrow H$. Therefore, $E^{p^{-\infty}} \simeq E \times_{X} X^{p^{-\infty}} \simeq E$ is an isomorphism.

Now, let $H^{\prime}$ be a smooth affine group scheme over $k$, and let $H=H^{p^{-\infty}}$ denote its perfection. Then $H$ is an affine group scheme. Let $X$ be a perfect algebraic space.

Lemma A.8. The functor $E^{\prime} \rightarrow{E^{\prime}}^{p^{-\infty}}$ is an equivalence of categories from the groupoid of $H^{\prime}$-torsors on $X$ to the groupoid of $H$-torsors on $X$. The quasi-inverse functor is given by push-out of an $H$-torsor along $\varepsilon: H \rightarrow H^{\prime}$, denoted by $E \mapsto E \times{ }^{H, \varepsilon} H^{\prime}$.

Proof. Given an $H$-torsor $E$, the natural map $E \rightarrow E \times{ }^{H, \varepsilon} H^{\prime}$ gives a morphism $E \rightarrow$ $\left(E \times{ }^{H, \varepsilon} H^{\prime}\right)^{p^{-\infty}}$ of $H$-torsors, and therefore is an isomorphism. Conversely, let $E^{\prime}$ be an $H^{\prime}$-torsor on $X$, and let $E=E^{p^{-\infty}}$ be the corresponding $H$-torsor. We want to show that $E \times{ }^{H, \varepsilon} H^{\prime} \simeq E^{\prime}$. As $H^{\prime}$ is smooth, we can trivialize $E^{\prime}$ by an étale cover $U \rightarrow X$ and therefore represents $E^{\prime}$ by a cocycle $c^{\prime}: U \times_{X} U \rightarrow H^{\prime}$. As $U \times_{X} U$ is perfect by Lemma A.2 the cocycle $c^{\prime}$ gives a cocycle $c: U \times_{X} U \rightarrow H$ by A.1.3 , which is nothing but the cocyle represents $E$. Then $E \times{ }^{H, \varepsilon} H^{\prime}$ is represented by the cocycle $U \times_{X} U \stackrel{c}{\rightarrow} H \stackrel{\varepsilon}{\rightarrow} H^{\prime}$, which is exactly $c^{\prime}$.

If $X$ is a space with an action of an affine group scheme $H$, we denote by $[X / H]$ the stack whose $R$-points are the groupoid of pairs $(E, \phi)$, where $E$ is an $H$-torsor on $\operatorname{Spec} R$, and $\phi: E \rightarrow Y$ is an $H$-equivariant morphism. Note that if the action is free, then $[X / H]$ is a $k$-space and the natural morphism $X \rightarrow[X / H]$ is an $H$-torsor.

We also recall the construction of the twisted product. Let $H$ be an affine group scheme and $E \rightarrow X$ be an $H$-torsor, and let $T$ be a space with an $H$-action, one can form the twisted product

$$
X \tilde{\times} T:=E \times{ }^{H} T=[E \times T / H],
$$

which is a space over $k$. Assume that $H$ is (the perfection of) an affine group scheme of finite type over $k$. Then if $X, T$ are (perfect) fpqc algebraic spaces, so is $X \tilde{\times} T$. Indeed, we can find an fppf cover $U \rightarrow X$ that trivializes $E^{17}$. Then $U \times T$ is an fppf cover of $X \tilde{\times} T$. Therefore, $X \tilde{\times} T$ is an algebraic space by [St, Tag04S5].

\footnotetext{
${ }^{17}$ If $H$ is of finite type, this is clearly. Otherwise, use Lemma A.26
} 
A.1.4. In fact in the paper we will only consider presheaves on the category perfect $k$ algebras.

Definition A.9. Let $\mathrm{Aff}_{k}^{\mathrm{pf}}$ be the opposite category of perfect $k$-algebras, endowed with the fpqc topology. A perfect space is a sheaf on $\mathrm{Aff}_{k}^{\mathrm{pf}}$ with respect to this topology.

The category of perfect $k$-spaces is denoted by $\mathrm{Sp}_{k}^{\mathrm{pf}}$. There is a natural functor $\mathrm{Sp}_{k} \rightarrow \mathrm{Sp}_{k}^{\mathrm{pf}}$ by restriction of a space $\mathcal{F}$ to $\mathrm{Aff}_{k}^{\mathrm{pf}}$. We denote the induced perfect space by $\mathcal{F}^{\mathrm{pf}}$. Note that if $X$ is an fpqc algebraic space, then $X^{\mathrm{pf}}=\left(X^{p^{-\infty}}\right)^{\mathrm{pf}}$.

The functor $\mathrm{Sp}_{k} \rightarrow \mathrm{Sp}_{k}^{\mathrm{pf}}$ is far from being faithful. However, we have the following crucial observation.

Lemma A.10. The the composition

$$
\operatorname{AlgSp}_{k}^{\mathrm{pf}} \subset \operatorname{AlgSp}_{k} \subset \mathrm{Sp}_{k} \rightarrow \mathrm{Sp}_{k}^{\mathrm{pf}} .
$$

is a full embedding.

Proof. Let $X$ and $Y$ be two perfect fpqc algebraic spaces. We need to show that

$$
\operatorname{Hom}_{\operatorname{AlgSp}_{k}^{\mathrm{pf}}}(X, Y)=\operatorname{Hom}_{\mathrm{Sp}_{k}^{\mathrm{pf}}}\left(X^{\mathrm{pf}}, Y^{\mathrm{pf}}\right) \text {. }
$$

Let $\left\{U_{i} \rightarrow X\right\}$ be a family of étale cover of $X$ by affine schemes, and let $\left\{V_{i j h} \rightarrow U_{i} \times_{X} U_{j}\right\}$ be a family of étale cover of $U_{i} \times_{X} U_{j}$ by affine schemes. By Lemma A.2, all $U_{i}$ and $V_{i j h}$ are perfect schemes. Therefore, by definition $\operatorname{Hom}_{\operatorname{AlgSp}_{k}^{\mathrm{pf}}}\left(U_{i}, Y\right)=\operatorname{Hom}_{\mathrm{Sp}_{k}^{\mathrm{pf}}}\left(U_{i}^{\mathrm{pf}}, Y^{\mathrm{pf}}\right)$, etc.

Note that (A.1.2) implies that the following sequence is an equalizer

$$
\operatorname{Hom}_{\operatorname{AlgSp}_{k}^{\mathrm{pf}}}(X, Y) \rightarrow \prod_{i} \operatorname{Hom}_{\mathrm{AlgSp}_{k}^{\mathrm{pf}}}\left(U_{i}, Y\right) \rightarrow \prod_{i j h} \operatorname{Hom}_{\operatorname{AlgSp}_{k}^{\mathrm{pf}}}\left(V_{i j h}, Y\right) .
$$

Likewise, the sequence

$$
\operatorname{Hom}_{\mathrm{Sp}_{k}^{\mathrm{pf}}}\left(X^{\mathrm{pf}}, Y^{\mathrm{pf}}\right) \rightarrow \prod_{i} \operatorname{Hom}_{\mathrm{Sp}_{k}^{\mathrm{pf}}}\left(U_{i}^{\mathrm{pf}}, Y^{\mathrm{pf}}\right) \rightarrow \prod_{i j h} \operatorname{Hom}_{\mathrm{Sp}_{k}^{\mathrm{pf}}}\left(V_{i j h}^{\mathrm{pf}}, Y^{\mathrm{pf}}\right)
$$

is also an equalizer (in fact, it is enough to use the injectivity of the first map). The lemma follows by comparing these two sequences.

Therefore, given a perfect space $\mathcal{F}$, or more generally a presheaf on $\mathrm{Aff}_{k}^{\mathrm{pf}}$, it makes sense to ask whether $\mathcal{F}$ is represented by a perfect algebraic space, and given a map $f: \mathcal{F} \rightarrow \mathcal{G}$ of perfect spaces, it makes sense to ask whether it is representable by perfect algebraic spaces. If a property $(\mathrm{P})$ of morphisms between algebraic spaces is stable under base change and is étale local on the source and target, then it makes sense to say whether a representable morphism $f: \mathcal{F} \rightarrow \mathcal{G}$ of perfect spaces has Property (P). For example, we can define open/closed immersions, étale morphisms, fpqc maps in $\mathrm{Sp}_{k}^{\mathrm{pf}}$, etc.

We can also define the notion of torsors in $\mathrm{Sp}_{k}^{\mathrm{pf}}$, just as $\mathrm{Sp}_{k}$. Let $H$ be a perfect affine group scheme. It gives an object $H^{\mathrm{pf}}$ in $\mathrm{Sp}_{k}^{\mathrm{pf}}$. If $X$ is a perfect space with a action of $H^{\mathrm{pf}}$, then we can define a stack $\left[X / H^{\mathrm{pf}}\right]$ on $\mathrm{Aff}_{k}^{\mathrm{pf}}$ as before and if the action is free, $\left[X / H^{\mathrm{pf}}\right]$ is also a perfect space. The natural map $X \rightarrow\left[X / H^{\mathrm{pf}}\right]$ is an $H^{\mathrm{pf}}$-torsor. Note that if $X$ is a perfect algebraic space, which gives $X^{\mathrm{pf}}$ in $\mathrm{Sp}_{k}^{\mathrm{pf}}$, then by Lemma A.10 giving an action of $H^{\mathrm{pf}}$ on $X^{\mathrm{pf}}$ is the same as giving an action of $H$ on $X$ and if the action is free, $\left[X^{\mathrm{pf}} / H^{\mathrm{pf}}\right]=[X / H]^{\mathrm{pf}}$.

We define an ind-perfect algebraic space as a perfect $k$-space that can be represented as an inductive limit $\left\{X_{i}\right\}$ of perfect fpqc algebraic spaces, such that $X_{i} \rightarrow X_{i+1}$ is a closed embedding.

In the sequel, the image of a perfect fpqc algebraic space $X$ in $\mathrm{Sp}_{k}^{\mathrm{pf}}$ is still denoted by $X$, as opposed to $X^{\mathrm{pf}}$ as above. However, for a general space $\mathcal{F}$, its image in $\mathrm{Sp}_{k}^{\mathrm{pf}}$ will be denoted by $\mathcal{F}^{\mathrm{pf}}$. 


\section{A.2. Perfect algebraic spaces perfectly of finite presentation.}

A.2.1. Perfect schemes/algebraic spaces of positive dimension are never of finite type over $k$. But we shall see the "infinity" here is really mild.

Definition A.11. We say that a perfect $k$-algebraic space $X$ is locally perfectly of finite type18 if there exist an étale affine cover $\left\{U_{i}\right\}$ of $X$ such that each $U_{i}$ is the perfection of an affine scheme of finite type over $k$. We say a perfect $k$-algebraic space $X$ is perfectly of finite type if it is locally perfectly of finite type and quasi-compact. We say that a perfect $k$-algebraic space perfectly of finite presentation ( $p f p$ for short) if it is perfectly of finite type and quasi-separated.

Remark A.12. (i) A pfp perfect algebraic space is an fpqc algebraic space. (ii) In [Se, a separated and perfectly of finite type perfect $k$-scheme is called a perfect variety.

We have the following result, generalizing [Se, §1.4, Proposition 9].

Proposition A.13. Let $X$ be a perfect algebraic space over $k$. Then $X$ is perfectly of finite presentation if and only if there exist an algebraic space $X^{\prime}$ of finite presentation over $k$ such that $X=X^{p^{-\infty}}$.

Proof. Clearly, if such $X^{\prime}$ exists, then $X$ is perfectly of finite presentation. We prove the converse. In fact, we prove a slightly stronger result.

Lemma A.14. Let $X$ be a perfect pfp algebraic space over $k$, and $U \subset X$ be an open dense subspace. Let $U^{\prime}$ be a reduced algebraic space of finite presentation over $k$ with $U=U^{\prime p^{-\infty}}$. Then there is $X^{\prime}$, of finite presentation over $k$, containing $U^{\prime}$ as an open subspace, such that $X=X^{p^{-\infty}}$.

For an algebraic space $S$, let $|S|$ denote its underlying topological space.

We first assume that $X$ is a scheme. We define a sheaf of rings on $|X|$ by

$$
\mathcal{O}_{X^{\prime}}=\left\{f \in \mathcal{O}_{X}|f|_{|U|} \in \mathcal{O}_{U^{\prime}}\right\} \text {. }
$$

In other words, Let $j:|U| \rightarrow|X|$ denote the inclusion of topological spaces. Then $\mathcal{O}_{X^{\prime}}:=$ $j_{*} \mathcal{O}_{U^{\prime}} \cap \mathcal{O}_{X}$, where the intersection is taken inside $j_{*} \mathcal{O}_{U}$. Rephrasing differently, $X^{\prime}$ is the push-out of $U^{\prime} \leftarrow U \hookrightarrow X$ in the category of locally ringed spaces. It is easy to check that the ringed space $\left(|X|, \mathcal{O}_{X^{\prime}}\right)$ is a scheme, of finite type over $k$, and $X=X^{p^{-\infty}}$.

We do not know whether this construction commute with étale base change. I.e. if we have the diagram $\tilde{U}^{\prime} \leftarrow \tilde{U} \hookrightarrow \tilde{X}$ étale over $U^{\prime} \leftarrow U \hookrightarrow X$, we do not know whether the corresponding push-out $\tilde{X}^{\prime}$ is étale over $X^{\prime}$. So the statement for schemes does not immediately implies the statement for algebraic spaces.

To proceed, we adapt the arguments of [CLO, Theorem 1.2.2] in our context (which is simpler). The key is the following fact. See [RG, Proposition 5.7.6] and [CLO, Theorem 3.1.1].

Proposition A.15. There is a stratification of $X$ by quasi-compact open subspaces $X=$ $U_{n} \supset U_{n-1} \supset \cdots \supset U_{1} \supset U_{0}=\emptyset$, such that for each $i>0$, there exists an étale cover $\varphi_{i}: V_{i} \rightarrow U_{i}$ by a quasi-compact, separated scheme $V_{i}$ with $\varphi_{i}$ restricting to an isomorphism over the closed subspace $Z_{i}=U_{i}-U_{i-1}$ in $U_{i}$ endowed with its reduced structure. Moreover, each $\varphi_{i}$ is separated, and each $Z_{i}$ is a separated and quasi-compact scheme.

In particular, we see that $V_{1} \rightarrow U_{1}$ is an isomorphism so $U_{1}$ is a separated scheme perfectly of finite presentation over $k$. Applying Lemma A.14 to the scheme $U_{1}$, with the given open subscheme $U_{1} \cap U$, we have $U_{1}=U_{1}^{\prime p^{-\infty}}$ for some reduced scheme $U_{1}^{\prime}$ of finite type over $k$, and $U_{1}^{\prime}$ contains $\left.U^{\prime}\right|_{\left|U \cap U_{1}\right|}$ as an open subscheme. We proceed by induction.

\footnotetext{
${ }^{18}$ The terminology is suggested by B. Conrad.
} 
Suppose we have $U_{i}=U_{i}^{\prime p^{-\infty}}$, where $U_{i}^{\prime}$ is an algebraic space of finite presentation over $k$, containing $\left.U^{\prime}\right|_{\left|U \cap U_{i}\right|}$ as an open subspace. Let $W_{i}=\varphi_{i+1}^{-1}\left(U_{i} \cup\left(U \cap U_{i+1}\right)\right) \subset V_{i+1}$. Note that $U_{i}^{\prime}$ and $\left.U^{\prime}\right|_{\left|U \cap U_{i+1}\right|}$ glue to an algebraic space, denoted by $\tilde{U}_{i}^{\prime}$, whose perfection is $U_{i} \cup\left(U \cap U_{i+1}\right)$. Then by Lemma A.2 $W_{i} \rightarrow U_{i} \cup\left(U \cap U_{i+1}\right)$ descends to an étale cover $W_{i}^{\prime} \rightarrow \tilde{U}_{i}^{\prime}$, where $W_{i}^{\prime}$ is of finite type over $k$. By Lemma A.14 for schemes, we can find $V_{i+1}^{\prime}$ with $V_{i+1}^{\prime}{ }^{-\infty}=V_{i+1}$ and $V_{i+1}^{\prime}$ contains $W_{i}^{\prime}$ as an open subscheme. Note that $\Delta: W_{i}^{\prime} \rightarrow W_{i}^{\prime} \times_{\tilde{U}_{i}^{\prime}} W_{i}^{\prime}$ is an open embedding. So we can define the scheme $R_{i+1}^{\prime}$ as the gluing of $V_{i+1}^{\prime}$ and $W_{i}^{\prime} \times_{\tilde{U}_{i}^{\prime}} W_{i}^{\prime}$ along $W_{i}^{\prime}$. It is of finite type over $k$, as both $V_{i+1}^{\prime}$ and $W_{i}^{\prime} \times_{U_{i}^{\prime}} W_{i}^{\prime}$ are of finite type. In addition, the two projections $W_{i}^{\prime} \times_{\tilde{U}_{i}^{\prime}} W_{i}^{\prime} \rightrightarrows W_{i}^{\prime}$ glue to $R_{i+1}^{\prime} \rightrightarrows V_{i+1}^{\prime}$, which clearly is an étale equivalence relation. In addition, it is clear that the perfection of the equivalence relation gives back to $V_{i+1} \times_{U_{i+1}} V_{i+1} \rightrightarrows V_{i+1}$. Let $U_{i+1}^{\prime}=V_{i+1}^{\prime} / R_{i+1}^{\prime}$ be the algebraic space, which is of finite presentation over $k$. Then $U_{i+1}=U_{i+1}^{\prime}{ }^{p^{-\infty}}$, containing $\left.U^{\prime}\right|_{\left|U \cap U_{i+1}\right|}$ as an open subspace.

Sometimes, we call such an $X^{\prime}$ as in Proposition A.13 a "model" or a "deperfection" of $X$.

Remark A.16. Let $X^{\prime}$ be the algebraic space constructed in Lemma A.14. Then it is easy to we see that for a scheme $Y$, the natural map $\operatorname{Hom}\left(X^{\prime}, Y\right) \rightarrow \operatorname{Hom}(X, Y) \times_{\operatorname{Hom}\left(U^{\prime}, Y\right)}$ $\operatorname{Hom}(U, Y)$ is a bijection. Indeed, if $X$ is a scheme, this follows from the fact that $X^{\prime}$ is the push-out of $U^{\prime} \leftarrow U \rightarrow X$. The case when $X$ is an algebraic space is reduced to the scheme case by using the étale cover $\tilde{X}=V_{n} \rightarrow X$ as in Proposition A.15. We do not know this is true if one replaces $Y$ by an algebraic space.

The following statement generalizes [Se, §1.4, Proposition 8].

Proposition A.17. Let $f: X \rightarrow Y$ be a morphism between perfect pfp algebraic spaces over $k$. Then there exists a morphism $f^{\prime}: X^{\prime} \rightarrow Y^{\prime}$ between algebraic spaces of finite presentation over $k$ such that $f=f^{\prime p^{-\infty}}$.

Proof. Let $X^{\prime}, Y^{\prime}$ be models of $X, Y$. Then there is a canonical map $\varepsilon: Y \rightarrow Y^{\prime}$. Recall that $\sigma: X^{\prime} \rightarrow X^{\prime}$ is affine by Lemma A.2. Then by a criterion of locally of finite presentation morphisms ([EGAIV] \$8.14], generalized in CLO, Proposition A.3.1], see also [St, Tag049I]), the map $\varepsilon f$ factors as $X \rightarrow X^{\prime(m)} \rightarrow Y^{\prime}$, where $X^{\prime(m)}=X^{\prime}$ with the $k$-structure given by $X^{\prime} \stackrel{\sigma^{m}}{\rightarrow} X^{\prime} \rightarrow$ Speck. Rename $X^{\prime(m)}$ as $X^{\prime}$, and we are done.

Definition A.18. Let $f: X \rightarrow Y$ be a morphism between two pfp perfect algebraic spaces over $k$. We say that $f$ is perfectly proper if it is separated and is universally closed. We say $X$ is perfectly proper if $X \rightarrow$ Speck is perfectly proper.

Lemma A.19. For a morphism $f: X \rightarrow Y$ between two pfp perfect algebraic spaces over $k$, $f$ is perfectly proper if and only if for every $f^{\prime}: X^{\prime} \rightarrow Y^{\prime}$ is as in LemmaA.17, $f^{\prime}$ is proper.

Proof. By Lemma A.6, $f$ is separated and universally closed if and only if so is $f^{\prime}$.

Proposition A.20. Let $f: X \rightarrow Y$ be a morphism between two pfp perfect algebraic spaces. Then $f$ is perfectly proper if and only if the valuative criterion holds for every perfect valuation ring $R$ over $k$.

Proof. In fact, if $f$ is perfectly proper, then it is the perfection of a proper morphism $f^{\prime}: X^{\prime} \rightarrow Y^{\prime}$. Therefore, the map $\operatorname{Spec} R \rightarrow Y \rightarrow Y^{\prime}$ lifts to $\operatorname{Spec} R \rightarrow X^{\prime}$. As $R$ is perfect, it factors through $\operatorname{Spec} R \rightarrow X$ by (A.1.3). To prove the converse, note that every perfect local ring $A$ in a perfect field $K$ is dominated by a perfect valuation ring. Then the usual arguments of valuative criterion for properness go through with obvious modifications.

We note that the perfection of a valuation ring is a valuation ring. 
Lemma A.21. Let $\mathcal{E}$ be a locally free sheaf of finite rank on a pfp perfect algebraic $X$ over $k$. Then there exists a model $\left(X^{\prime}, \mathcal{E}^{\prime}\right)$ of $(X, \mathcal{E})$, i.e algebraic space $X^{\prime}$, of finite presentation over $k$, and a locally free sheaf $\mathcal{E}^{\prime}$ of finite rank such that $(X, \mathcal{E})=\left(X^{p^{-\infty}}, \varepsilon^{*} \mathcal{E}^{\prime}\right)$, where $\varepsilon: X \rightarrow X^{\prime}$ is the tautological map.

Proof. Let $\varepsilon: X \rightarrow X^{\prime}$ be a model. As $X$ is quasi compact, we can find a finite étale cover $\left\{U_{i}\right\}$ of $X$ such that $\left.\mathcal{E}\right|_{U_{i}} \simeq \mathcal{O}_{U_{i}}^{r}$. Then we obtain a Čech cocycle $f_{i j}: U_{i j}:=U_{i} \times_{X} U_{j} \rightarrow$ $\mathrm{GL}_{r}$. By Proposition A.4 the étale cover $\left\{U_{i}\right\}$ descend to an étale cover $\left\{U_{i}^{\prime}\right\}$ of $X^{\prime}$. Let $U_{i j}^{\prime}=U_{i}^{\prime} \times_{X^{\prime}} U_{j}^{\prime}$, and $U_{i j k}^{\prime}=U_{i}^{\prime} \times_{X^{\prime}} U_{j}^{\prime} \times_{X^{\prime}} U_{k}^{\prime}$. The map $f_{i j}$ factors as $f_{i j}^{\prime}: U_{i j}^{\prime(m)} \rightarrow \mathrm{GL}_{r}$ for some $m$ large enough, where as in Lemma A.17 $U_{i j}^{\prime(m)}=U_{i j}^{\prime}$ with the $k$-structure given by $U_{i j}^{\prime} \stackrel{\sigma^{m}}{\rightarrow} U_{i j}^{\prime} \rightarrow$ Speck. Let $h_{i j k}=f_{i j}^{\prime} f_{j k}^{\prime} f_{k i}^{\prime}: U_{i j k}^{(m)} \rightarrow \mathrm{GL}_{r}$. Then $\varepsilon^{*} h_{i j k}=1: U_{i j k} \rightarrow \mathrm{GL}_{r}$. Then there is some $n$ big enough such that $\left(\sigma^{n}\right)^{*} h_{i j k}=1$ for all $i, j, k$. Therefore, the vector bundle $\mathcal{E}$ can be defined over $X^{\prime(m+n)}$ with Čech cocyle (with respect to the étale cover $\left.\left\{U_{i}^{\prime(m+n)}\right\}\right)$ with given by $\left(\sigma^{n}\right)^{*} f_{i j}^{\prime}$.

Corollary A.22. Let $X$ be a perfect algebraic space over $k$. Let $\mathcal{E}$ be a locally free sheaf of rank $n$ over $X$. Then the perfect space which assigns every $f: \operatorname{Spec} R \rightarrow X$ the rank $i$ quotient $f^{*} \mathcal{E} \rightarrow \mathcal{Q} \rightarrow 0$ is represented by a perfect algebraic space $\mathrm{Gr}^{p^{-\infty}}(i, \mathcal{E})$ perfectly proper over $X$. In particular, if $X$ is perfectly proper, so is $\operatorname{Gr}^{p^{-\infty}}(i, \mathcal{E})$.

Proof. Let $\left(X^{\prime}, \mathcal{E}^{\prime}\right)$ be as in Lemma A.21 Then $\mathrm{Gr}^{p^{-\infty}}(i, \mathcal{E})$ is the perfection of the usual Grassmannian $\operatorname{Gr}\left(i, \mathcal{E}^{\prime}\right)$ of rank $i$ quotients of $\mathcal{E}^{\prime}$.

In the sequel, we denote $\operatorname{Gr}^{p^{-\infty}}(i, \mathcal{E})$ by $\operatorname{Gr}^{p^{-\infty}}(i, n)$ if $X=$ Speck and $\mathcal{E}=k^{n}$ is the trivial $n$-dimensional vector space. We denote $\operatorname{Gr}^{p^{-\infty}}(1, \mathcal{E})$ by $\mathbb{P}^{p^{-\infty}}(\mathcal{E})$ and $\mathrm{Gr}^{p^{-\infty}}(1, n+1)$ by $\mathbb{P}^{n, p^{-\infty}}$, the perfect projective space.

Remark A.23. On $\mathbb{P}^{n, p^{-\infty}}$, there is the following tautogolical rank one quotient $V \otimes \mathcal{O}_{\mathbb{p} n, p-\infty} \rightarrow$ $\mathcal{O}_{\mathbb{P}^{n, p^{-}}}(1)$, and therefore a distinguished element $\mathcal{O}_{\mathbb{P} n, p^{-}}(1)$ (or $\mathcal{O}(1)$ for simplicity) is in $\operatorname{Pic}\left(\mathbb{P}^{n, p^{-\infty}}\right)$. However, $\mathcal{O}(1)$ is not the generator of the Picard group. Namely there exists the invertible sheaf $\mathcal{O}(1 / p)=\left(\sigma^{-1}\right)^{*} \mathcal{O}(1)$, and the Picard group is isomorphic to $\mathbb{Z}[1 / p]$.

In the paper, we will use the terminology "locally free sheaf" (resp. "invertible sheaf") on "vector bundle" (resp. "line bundle") interchangably.

We will also need the following definition.

Definition A.24. Let $f: X \rightarrow Y$ be a map between two pfp algebraic spaces. We say that $f$ is perfectly smooth at $x \in X$ if there exist étale $U \rightarrow X$ atlas at $x$ and $V \rightarrow Y$, such that $U \rightarrow Y$ factors as $U \stackrel{h}{\rightarrow} V \rightarrow Y$ and $h$ factors as $h=$ pr $\circ h^{\prime}$, where $h^{\prime}: U \rightarrow V \times\left(\mathbb{A}^{n}\right)^{p^{-\infty}}$ is étale and pr: $V \times\left(\mathbb{A}^{n}\right)^{p^{-\infty}} \rightarrow V$ is the projection. We say that $f$ is perfectly smooth if it is perfectly smooth at every point of $X$. We say that $X$ is perfectly smooth (at $x$ ) if $X \rightarrow$ Speck is perfectly smooth (at $x$ ).

Note that every pfp perfect algebraic space $X$ contains a perfectly smooth open dense subspace.

A.2.2. We need to construct the quotient in some cases for an action of a perfect group scheme on a perfect scheme. In [Se], a perfect group variety is defined as a group object in the category of perfect varieties.

Lemma A.25. Let $H$ be a separated perfect pfp group scheme over $k$. Then there exists a smooth algebraic group $H_{0}$ over $k$ such that $H=H_{0}^{p^{-\infty}}$. 
Proof. A priori, $H$ is the perfection of a scheme of finite type over $k$. But as is shown in [Se, $\S 1.4$, Proposition 10], $H$ is the perfection of a group scheme $H^{\prime}$ of finite type over $k$. Let $H_{0}=H^{\prime}$ red be the reduced subscheme. As $k$ is a perfect field, $H_{0}$ is closed subgroup scheme of $H^{\prime}$ and is smooth. In addition, $H_{0}^{p^{-\infty}}=H$.

Corollary A.26. Let $H$ be as above. Then every $H$-torsor on a perfect algebraic space $X$ can be trivialized étale locally on $X$.

Proof. This is the combination of Lemma A.25 and Lemma A.8.

Lemma A.27. Let $H$ be an affine perfect pfp scheme over $k$ acting on a perfect php scheme $X$ over $k$. Then there exists a smooth affine algebraic group $H^{\prime}$ of finite type over $k$, acting on an algebraic space $X^{\prime}$ of finite presentation over $k$, whose perfection gives the original action map.

Proof. Recall the following basic fact: let $H$ be an affine group scheme over $k$, with ring of functions $A$. Let $\rho: V \rightarrow A \otimes_{k} V$ be a representation of $H$. Then $V$ is the union of finite dimensional $H$-modules.

Now let $\eta_{1}, \ldots, \eta_{n}$, denote the generic points of $|X|$, with the residue fields $k\left(\eta_{i}\right)$, which are the perfection of a finitely generated field over $k$. Let $F_{i} \subset k\left(\eta_{i}\right)$ be a subfield finitely generated over $k$. Let $B_{0}=\prod F_{i} \subset B=\prod k\left(\eta_{i}\right)$. Let $V_{0}$ is a finite dimensional $k$-subspace of $B_{0}$ that generate $B_{0}$ (by additions, multiplications, and fractions) and let $V^{\prime}$ be a finite dimensional $H$-invariant subspace of $B$ that containing $V_{0}$. Let $B^{\prime} \subset B$ be the subalgebra generated by $V^{\prime}$ (by additions, multiplications, and fractions). Then $B^{\prime}$ is an $H$-invariant subalgebra of $B$ and $B^{p^{-\infty}}=B$ (since $B^{p^{-\infty}} \supset B_{0}^{p^{-\infty}}=B$ ). Using the same formula as (A.2.1) to define a scheme $X^{\prime}$ whose perfection is $X$, and the product of the residue fields of whose generic points is $B^{\prime}$. Then $X^{\prime}$ is of finite type that admits an action of $H$, which induces the action of $H$ on $X$. As $H=\lim _{0} H_{0}$, the map $H \times X^{\prime} \rightarrow X^{\prime}$ is induced from some $H^{\prime} \times X^{\prime} \rightarrow X^{\prime}$.

Proposition A.28. Let $H, X$ be as above. Furthermore, we assume that the action is free, i.e. map $a=\left(\right.$ act, $\left.\mathrm{pr}_{2}\right): H \times X \rightarrow X \times X,(g, x) \mapsto(g x, x)$ is a monomorphism. Then $[X / H]$ is represented by a perfect pfp algebraic space $X / H$ over $k$. In addition, if $X$ is separated and $a$ is a closed embedding, then $[X / H]$ is separated as well.

Proof. First note that in order to prove this proposition, we can replace $X$ by an $H$ equivariant étale cover $Y \rightarrow X$ by a perfect pfp algebraic space $Y$ (not necessarily a scheme). Indeed, If $[Y / H]$ is representable by a perfect php algebraic space over $k$, then by Lemma A.26. we can find an étale cover of $[Y / H]$ by an affine scheme that trivializes the $H$-torsor $Y \rightarrow[Y / H]$. I.e. after further étale localization, we can assume $Y=H \times U$. Then clearly $[X / H]$ is representable if such $Y$ exists.

By Lemma A.27 we have act' $: H^{\prime} \times X^{\prime} \rightarrow X^{\prime}$ that induces act : $H \times X \rightarrow X$. Now the action may not be free, but it is quasi-finite. There exists an $H^{\prime}$-equivariant étale cover $Y^{\prime} \rightarrow X^{\prime}$ by a separated algebraic space $Y^{\prime}$ of finite presentation, such that there exists $H^{\prime}$ equivariant finite flat morphism $Z^{\prime} \rightarrow Y^{\prime}$ and $Z^{\prime}$ is an $H^{\prime}$-torsor over some quasi-projective scheme $\mathfrak{Z}^{\prime}$ over $k$. Indeed, as the Artin stack $\mathfrak{X}^{\prime}:=\left[X^{\prime} / H^{\prime}\right]$ is of finite presentation and has quasi-finite diagonal, by [KM, Lemma 3.3, Proposition 4.2] or [Co, Lemma 2.1, 2.2], there exists an étale cover $\mathfrak{Y}^{\prime} \rightarrow \mathfrak{X}^{\prime}$ by an Artin stack $\mathfrak{Y}^{\prime}$ separated and of finite presentation over $k$, such that there exists a finite flat cover $\mathfrak{Z}^{\prime} \rightarrow \mathfrak{Y}^{\prime}$ with $\mathfrak{Z}^{\prime}$ being a quasi-projective scheme over $k$. Then pulling back $\mathfrak{Z}^{\prime} \rightarrow \mathfrak{Y}^{\prime} \rightarrow \mathfrak{X}^{\prime}$ along $X^{\prime} \rightarrow \mathfrak{X}^{\prime}$ gives $Z^{\prime} \rightarrow Y^{\prime} \rightarrow X^{\prime}$. Now we can prove our statement for $Y=Y^{\prime p^{-\infty}}$ instead of $X$. Note that in this case $H^{\prime} \times Y^{\prime} \rightarrow Y^{\prime} \times Y^{\prime}$ is in fact finite.

It is known that in this case, the coarse moduli space of $\mathfrak{Y}^{\prime}$ exists as a separated scheme of finite type over $k$, denoted by $Y^{\prime} / H^{\prime}$. Indeed, Let $V=\mathfrak{Z} \times_{\mathfrak{Y}} \mathfrak{Z}$. Then $Y^{\prime} / H^{\prime}$ is the 
coequalizer of $V \rightrightarrows \mathfrak{Z}$ in the category of locally ringed spaces. See [SGA4, V, 4.1] and [Co $\S 3]$.

We have $H^{\prime} \times Y^{\prime} \rightarrow Y^{\prime} \times{ }_{Y^{\prime} / H^{\prime}} Y^{\prime} \rightarrow Y^{\prime} \times Y^{\prime}$, where the second map is a closed embedding (since $Y^{\prime} / H^{\prime}$ is separated), and the first map is finite. Passing to the perfection, we see that $H \times Y \simeq Y_{\left(Y / H^{\prime}\right)^{p^{-\infty}} Y}$. I.e. $Y \rightarrow\left(Y^{\prime} / H^{\prime}\right)^{p^{-\infty}}$ is an $H$-torsor. Therefore we obtain a map $\left(Y^{\prime} / H^{\prime}\right)^{p^{-\infty}} \rightarrow[Y / H]$ which is clearly an isomorphism.

\section{A.3. $\ell$-adic sheaves.}

A.3.1. The notion of (constructible) étale sheaves is well defined for separated pfp perfect algebraic spaces. Indeed, let $X$ be such an algebraic space, and let $\varepsilon: X \rightarrow X^{\prime}$ be a model of $X$. Note that $\varepsilon$ is a universal homeomorphism, and by Lemma A.4 for an étale sheaf $\mathcal{F}$ on $X$ and an étale sheaf $\mathcal{F}^{\prime}$ on $X^{\prime}$, the natural maps

$$
\varepsilon^{*} \varepsilon_{*} \mathcal{F} \rightarrow \mathcal{F}, \quad \mathcal{F}^{\prime} \rightarrow \varepsilon_{*} \varepsilon^{*} \mathcal{F}^{\prime}
$$

are isomorphisms. In particular, for such $X$, one can define the corresponding $\ell$-adic derived category $D_{c}^{b}\left(X, \overline{\mathbb{Q}}_{\ell}\right)(\ell \neq p)$ as usual, with a pair of adjoint functors that are equivalences

$$
\varepsilon^{*}: D_{c}^{b}\left(X^{\prime}, \overline{\mathbb{Q}}_{\ell}\right) \simeq D_{c}^{b}\left(X, \overline{\mathbb{Q}}_{\ell}\right): \varepsilon_{*} .
$$

Given $f: X \rightarrow Y$, one can define six operations associated to $f$, thanks to Lemma A.17. The usual proper base change or smooth base change holds for perfectly proper or perfectly smooth maps. The notation of perverse sheaves work in this setting without change. In particular, we have the notion of the Goresky-Macpherson intermediate extension, and for every $X$, the intersection cohomology sheaf $\mathrm{IC}_{X}$. The restriction of $\mathrm{IC}_{X}$ to any perfectly smooth open subset $U$ is canonically isomorphic to $\overline{\mathbb{Q}}_{\ell}[2 \operatorname{dim} X](\operatorname{dim} X)$. We will denote by $\mathrm{P}(X)$ the category of perverse sheaves on $X$.

A.3.2. Let $X$ be a separated pfp perfect algebraic space over $k$. One can define the Chern classes for locally free sheaves on $X$ as usual. For $\mathcal{L}$ an invertible sheaf on $X$, corresponding to a class $[\mathcal{L}] \in H^{1}\left(X, \mathbb{G}_{m}\right)$, we define its Chern class $c_{1}(\mathcal{L})$ as its image under $H^{1}\left(X, \mathbb{G}_{m}\right) \rightarrow$ $H^{1}\left(X_{\bar{k}}, \mathbb{G}_{m}\right) \rightarrow H^{2}\left(X_{\bar{k}}, \mu_{\ell^{n}}\right)$. In general, if $\mathcal{E}$ is a locally free sheaf of rank $n$ over $X$, let $\mathcal{O}(1)=\mathcal{O}_{\mathbb{P}^{p^{-\infty}}(\mathcal{E})}(1)$ denote the tautological line bundle on $\mathbb{P}^{p^{-\infty}}(\mathcal{E})$ and let $\xi=c_{1}(\mathcal{O}(1)) \in$ $H^{2}\left(\mathbb{P}^{p^{-\infty}}(\mathcal{E})_{\bar{k}}, \mu_{\ell^{n}}\right)$. Then there are unique $c_{i}(\mathcal{E}) \in H^{2 i}\left(X_{\bar{k}}, \mu_{\ell^{n}}^{\otimes i}\right)$ such that

$$
\xi^{n}-c_{1}(\mathcal{E}) \xi^{n-1}+\cdots+(-1)^{n} c_{n}(\mathcal{E})=0,
$$

Passing to inverse limit and inverting $\ell$, we get $\overline{\mathbb{Q}}_{\ell}$-coefficient Chern classes. The usual properties of Chern classes hold in these settings.

One can also define the characteristic classes for general principal homogeneous spaces. Let $G$ be a (connected) reductive group over $k$ and let $G_{\overline{\mathbb{Q}}_{\ell}}$ be the corresponding split group over $\overline{\mathbb{Q}}_{\ell}$. Let $R_{G, \ell}=\operatorname{Sym}\left(\mathfrak{g}_{\overline{\mathbb{Q}}_{\ell}}^{*}(-1)\right)^{G_{\overline{\mathbb{Q}}_{\ell}}}$ be the algebra of invariant polynomials on the Lie algebra $\mathfrak{g}_{\overline{\mathbb{Q}}_{\ell}}(1)$. Then given a $G$-torsor $E$ on $X$ (equivalently a $G^{p^{-\infty}}$-torsor on $X$ by $\sqrt{\text { A.1.3) }}$, its characteristic classes can be regarded as a ring homomorphism

$$
c(E): R_{G, \ell} \rightarrow \mathrm{H}^{*}\left(X_{\bar{k}}, \overline{\mathbb{Q}}_{\ell}\right),
$$

which can be constructed as follows: Let $B$ be a Borel subgroup of $G_{\bar{k}}$, with the unipotent radical $U$ and $T=B / U$. Let $W$ be the Weyl group. Then the $T$-torsor $E_{\bar{k}} / U \rightarrow E_{\bar{k}} / B$ induces $c\left(E_{\bar{k} / U}\right): R_{T, \ell} \rightarrow \mathrm{H}^{*}\left(E_{\bar{k}} / B, \overline{\mathbb{Q}}_{\ell}\right)$ via the above construction of the Chern classes. Passing to some models, we see that $R_{G, \ell}=R_{T, \ell}^{W}$ maps to $\mathrm{H}^{*}\left(X_{\bar{k}}, \overline{\mathbb{Q}}_{\ell}\right) \subset \mathrm{H}^{*}\left(E_{\bar{k}} / B, \overline{\mathbb{Q}}_{\ell}\right)$, giving the desired (A.3.2). If $G$ is a general connected algebraic group, and let $G^{\text {red }}$ denote its reductive quotient. Then a $G$-torsor $E$ gives a $G^{\text {red }}$-torsor $E^{\text {red }}$, and we denote by $c(E)$ for $c\left(E^{\text {red }}\right)$. 
Remark A.29. Alternatively, one can define the Chern classes (or general characteristic classes) of $E \rightarrow X$ by passing to some model $E^{\prime} \rightarrow X^{\prime}$ (LemmaA.21) and define $c(E)=c\left(E^{\prime}\right)$ using the identification $\mathrm{H}^{*}\left(X_{\bar{k}}, \overline{\mathbb{Q}}_{\ell}\right)=\mathrm{H}^{*}\left(X_{\bar{k}}^{\prime}, \overline{\mathbb{Q}}_{\ell}\right)$. Then one shows that this definition is independent of the choice of the model.

A.3.3. We can also define the cycle class map in the current setting. First, if $X$ is irreducible of dimension $d$, there is a canonical isomorphism

$$
c_{X}: \mathrm{H}_{c}^{2 d}\left(X_{\bar{k}}, \overline{\mathbb{Q}}_{\ell}(d)\right) \simeq \overline{\mathbb{Q}}_{\ell},
$$

given as follows: Choose a model $\varepsilon: X \rightarrow X^{\prime}$, which induces the canonical isomorphism

$$
\varepsilon^{*}: \mathrm{H}_{c}^{2 d}\left(X_{\bar{k}}^{\prime}, \overline{\mathbb{Q}}_{\ell}(d)\right) \simeq \mathrm{H}_{c}^{2 d}\left(X_{\bar{k}}, \overline{\mathbb{Q}}_{\ell}(d)\right) .
$$

Then we define $c_{X}=c_{X^{\prime}} \circ\left(\varepsilon^{*}\right)^{-1}$. Note that if $f: X^{\prime} \rightarrow X^{\prime \prime}$ is a morphism of two $d$-dimensional irreducible algebraic spaces of finite type, the canonical isomorphism $f^{*}$ : $\mathrm{H}_{c}^{2 d}\left(X_{\bar{k}}^{\prime \prime}, \overline{\mathbb{Q}}_{\ell}(d)\right) \simeq \mathrm{H}_{c}^{2 d}\left(X_{\bar{k}}^{\prime}, \overline{\mathbb{Q}}_{\ell}(d)\right)$ is compatible with $c_{X^{\prime}}$ and $c_{X^{\prime \prime}}$. Therefore, $c_{X}$ is welldefined. Alternatively, one can build $c_{X}$ directly, starting from the canonical isomorphism

$$
c_{\mathbb{P}^{1, p^{-}}}: \mathrm{H}^{2}\left(\mathbb{P}_{\bar{k}}^{1, p^{-\infty}}, \mu_{\ell^{n}}\right) \simeq \operatorname{coker}\left(\operatorname{Pic}\left(\mathbb{P}^{1, p^{-\infty}}\right) \stackrel{\ell^{n}}{\rightarrow} \operatorname{Pic}\left(\mathbb{P}^{1, p^{-\infty}}\right)\right) \simeq \mathbb{Z} / \ell^{n}
$$

and then using functoriality of the six operations.

Now let $X$ be a perfect algebraic space essentially of finite type. Let $\omega_{X}=f ! \overline{\mathbb{Q}}_{\ell}$ denote the dualizing sheaf. We define the Borel-Moore homology of $X$ as

$$
\mathrm{H}_{i}^{\mathrm{BM}}\left(X_{\bar{k}}\right)=\mathrm{H}^{-i}\left(X_{\bar{k}}, \omega_{X}(-i / 2)\right) \text {. }
$$

The usual properties of the Borel-Moore homology hold (by the functoriality of the six operations). We list a few.

- If $f: X \rightarrow Y$ is a perfectly proper morphism, there is an canonical map

$$
f_{*}: \mathrm{H}_{*}^{\mathrm{BM}}\left(X_{\bar{k}}\right) \rightarrow \mathrm{H}_{*}^{\mathrm{BM}}\left(Y_{\bar{k}}\right) .
$$

- There is the canonical isomorphism $\mathrm{H}_{i}^{\mathrm{BM}}\left(X_{\bar{k}}\right) \simeq \mathrm{H}_{c}^{i}\left(X_{\bar{k}}, \overline{\mathbb{Q}}_{\ell}(i / 2)\right)^{*}$ and therefore if $X$ is irreducible of dimension $d$, there exists the fundamental class $[X]:=c_{X} \in$ $\mathrm{H}_{2 d}^{\mathrm{BM}}\left(X_{\bar{k}}\right)$. In general, if $X$ is $d$-dimensional, with $X_{1}, \ldots, X_{n}$ its irreducible components of dimension $d$, then the natural map $\bigoplus_{i} \mathrm{H}_{2 d}^{\mathrm{BM}}\left(\left(X_{i}\right)_{\bar{k}}\right) \simeq \mathrm{H}_{2 d}^{\mathrm{BM}}\left(X_{\bar{k}}\right)$ is an isomorphism. We denote by $[X]=\sum_{i}\left[X_{i}\right]$.

- If $X$ is irreducible, and essentially smooth, the fundamental class $[X]$, regarded as a map of sheaves $\overline{\mathbb{Q}}_{\ell} \rightarrow \omega_{X}[-2 d](-d)$ is an isomorphism. Therefore, $\mathrm{H}_{i}^{B M}\left(X_{\bar{k}}\right) \simeq$ $\mathrm{H}^{2 d-i}\left(X_{\bar{k}}, \overline{\mathbb{Q}}_{\ell}(d-i / 2)\right)$.

Finally, let $Z$ be a closed subset of codimension $r$. We denote the cycle $\operatorname{class} \operatorname{cl}(Z)$ of $Z$ as the image of $[Z]$ in $\mathrm{H}_{2(d-r)}^{\mathrm{BM}}\left(X_{\bar{k}}\right)$. If $X$ is essentially smooth and proper, we can regard $\operatorname{cl}(Z)$ as a class in $\mathrm{H}^{2 r}\left(X_{\bar{k}}, \overline{\mathbb{Q}}_{\ell}(r)\right)$.

A.3.4. We have the Lefschetz trace formula: Let $\mathcal{F}$ be an $\ell$-adic complex with constructible cohomology on a separated pfp perfect algebraic space $X$ over $\mathbb{F}_{q}$. As usual, one can attach a function

$$
f_{\mathcal{F}}: X\left(\mathbb{F}_{q}\right) \rightarrow \overline{\mathbb{Q}}_{\ell}, \quad x \mapsto \operatorname{tr}\left(\sigma_{x}, \mathcal{F}_{\bar{x}}\right)=\sum_{i}(-1)^{i} \operatorname{tr}\left(\sigma_{x},\left(\mathcal{H}^{i} \mathcal{F}\right)_{\bar{x}}\right),
$$

where $x \in X\left(\mathbb{F}_{q^{r}}\right), \bar{x}$ a geometric point over $x,\left(\mathcal{H}^{i} \mathcal{F}\right)_{\bar{x}}$ the stalk cohomology of $\mathcal{F}$ at $\bar{x}$, and $\sigma_{x}$ is the geometric Frobenius at $x$.

Let $f: X \rightarrow Y$ be a morphism of separated pfp perfect algebraic spaces over $\mathbb{F}_{q}$. Let $\mathcal{F}$ be an $\ell$-adic complex with constructible cohomolgy $X$. Then the trace formula gives

$$
f_{f_{!} \mathcal{F}}(y)=\sum_{x \in f^{-1}(y)\left(\mathbb{F}_{q}\right)} f_{\mathcal{F}}(x)
$$


Indeed, in order to prove this we can replace $f: X \rightarrow Y$ by a model $f^{\prime}: X^{\prime} \rightarrow Y^{\prime}$, and replace $\mathcal{F}$ by $\varepsilon_{*} \mathcal{F}$.

A.3.5. In the paper, we also need some basic facts about equivariant category and equivariant cohomology. Let $J$ be an affine pfp perfect group scheme over $k$. Recall that by Lemma A.25, $J$ is perfectly smooth. Let $X$ be a separated pfp perfect algebraic space over $k$ with an action of $J$. Then it make sense to talk about the $J$-equivariant perverse sheaves on $X$, denoted by $\mathrm{P}_{J}(X) 19$. I.e., an object in $\mathrm{P}_{J}(X)$ is a perverse sheaf on $X$ together with an isomorphism along the two maps $J \times X \rightrightarrows X$, satisfying the usual compatibility conditions.

We have the following two properties of the equivariant category. Let $J_{1} \subset J$ be a closed normal subgroup.

(i). If the action of $J_{1}$ on $X$ is free and $\left[X / J_{1}\right]$ is represented by an algebraic space $\bar{X}$, then the pull back along $q: X \rightarrow \bar{X}$ induces an equivalence of categories

$$
q^{*}\left[\operatorname{dim} J_{1}\right]: \mathrm{P}_{J / J_{1}}(\bar{X}) \simeq \mathrm{P}_{J}(X)
$$

(ii). If the action of $J_{1}$ on $X$ is trivial, and $J_{1}$ is the perfection of some unipotent group $J_{1}^{\prime}$. Then the forgetful functor

$$
\mathrm{P}_{J}(X) \rightarrow \mathrm{P}_{J / J_{1}}(X)
$$

is an equivalence of categories.

To prove (i), one can assume that $X=\bar{X} \times J_{1}$. Then the claim is easy. (ii) follows from $\mathrm{H}^{*}\left(J_{1}, \overline{\mathbb{Q}}_{\ell}\right)=\overline{\mathbb{Q}}_{\ell}$.

Let $\mathcal{A} \in \mathrm{P}_{J}(X)$, it makes sense to talk about the $J$-equivariant cohomology $\mathrm{H}_{J}^{*}\left(X_{\bar{k}}, \mathcal{A}\right)$. Namely, let $J_{0}$ be a smooth model of $J$ as in Lemma A.25. Let $\left\{E_{n} \rightarrow B_{n}\right\}$ denote a sequence of $J_{0}$-torsors over $\left\{B_{n}\right\}$, which approximates of the classifying space of $J_{0}$ in the sense that $B_{n} \subset B_{n+1}$ is a closed embedding, and $\mathrm{H}^{*}\left(\lim _{n} B_{n}\right)=\mathrm{H}^{*}\left(B J_{0}\right)$. E.g. we can embed $J_{0}$ into some $\mathrm{GL}_{r}$ such that $\mathrm{GL}_{r} / J_{0}$ is quasi-affine. Then for $n$ large, let $E_{n}:=S_{n, r}$ be the Stiefel variety, i.e. the tautological $\mathrm{GL}_{r}$-torsor over $\operatorname{Gr}(r, n)$. Then $B_{n}:=E_{n} / J_{0}$ is represented by a scheme, and the ind-scheme $\underline{\longrightarrow}_{n} B_{n}$ satisfies the required property. Then the sheaf $\overline{\mathbb{Q}}_{\ell} \otimes \mathcal{A}$ on $E_{n}^{p^{-\infty}} \times X$ is $J$-equivariant with respect to the diagonal action, and therefore by (A.3.3), descends to $\overline{\mathbb{Q}}_{\ell} \tilde{\otimes} \mathcal{A}$ on $E_{n} \tilde{\times} X$, which is a separated perfect pfp algebraic space by the discussion in A.1.3 Then

$$
\mathrm{H}_{J}^{*}\left(X_{\bar{k}}, \mathcal{A}\right):=\mathrm{H}^{*}\left(\underline{\lim }_{n}\left(E_{n}^{p^{-\infty}} \tilde{\times} X\right)_{\bar{k}}, \overline{\mathbb{Q}}_{\ell} \tilde{\otimes} \mathcal{A}\right) .
$$

From the construction $\mathrm{H}_{J}^{*}\left(X_{\bar{k}}, q^{*} \mathcal{A}\right)$ is a module over $\mathrm{H}^{*}\left(\lim _{n} B_{n}\right)=\mathrm{H}^{*}\left(B J_{0}\right)$. Let us also recall the Lie theoretical description of $\mathrm{H}^{*}\left(B J_{0}\right)$ in the case when $J_{0}$ is connected. Let $G=J_{0}^{\text {red }}$ be the reductive quotient of $J_{0}$ over $k$. Then $\mathrm{H}^{*}\left(B J_{0}\right)=R_{G, \ell}$.

It is clear from the definition that if $J_{1} \subset J$ acts freely on $X$ with $\bar{X}$ the quotient as above, then

$$
\mathrm{H}_{J}^{*}\left(X_{\bar{k}}, q^{*} \mathcal{A}\right)=\mathrm{H}_{J / J_{1}}^{*}\left(\bar{X}_{\bar{k}}, \mathcal{A}\right)
$$

If $J_{1}$ acts trivially on $X$ and $J_{1}$ is the perfection of a unipotent group, then

$$
\mathrm{H}_{J}^{*}\left(X_{\bar{k}}, \mathcal{A}\right)=\mathrm{H}_{J / J_{1}}^{*}\left(X_{\bar{k}}, \mathcal{A}\right) .
$$

If $J$ is a perfect affine group scheme with an action of $J$ on $X$, satisfying the following condition (which is always the case in the paper): there exists a closed normal subgroup $J_{1} \subset J$ of finite codimension acting trivially on $X$, and $J_{1}$ is the perfection of a pro-unipotent pro-algebraic group. Then we can define $\mathrm{P}_{J}(X)$ as $\mathrm{P}_{J / J_{1}}(X)$ and $\mathrm{H}_{J}^{*}(X, \mathcal{A})$ for $\mathcal{A} \in \mathrm{P}_{J}(X)$ as $\mathrm{H}_{J / J_{1}}^{*}(X, \mathcal{A})$. By (A.3.4) (A.3.6), these are independent of the choice of $J_{1} \subset J$.

\footnotetext{
${ }^{19}$ One can also define the equivariant derived category. But we do not need to use it in the paper.
} 


\section{Appendix B. More on mixed Characteristic AfFine Grassmannians}

In this section, we discuss some unsolved questions related to mixed characteristic affine Grassmannians. We omit most of the proofs. We also give an example of our construction.

B.1. The determinant line bundle. We continue to use the notations as in \$1. Recall that in equal characteristic setting, there is the important determinant line bundle $\mathcal{L}_{\text {det }}^{b}$ on $\mathrm{Gr}^{b}$. Its restriction to $\overline{\mathrm{Gr}}_{N}$ (equal characteristic analogue of $\overline{\mathrm{Gr}}_{N}$ ) is given as follows: for $(\mathcal{E}, \beta) \in{\overrightarrow{\mathrm{Gr}_{N}}}_{N}$ so that $\mathcal{E}_{0} / \mathcal{E}$ is a projective $R$-module of rank $N$, the fiber of $\mathcal{L}_{\text {det }}^{b}$ at this point is $\wedge^{N}\left(\mathcal{E}_{0} / \mathcal{E}\right)$. In mixed characteristic setting, as $\mathcal{E}_{0} / \mathcal{E}$ is not an $R$-module, the above formula does not make sense. Alternatively, one can try to define this line bundle by taking a filtration of $\mathcal{E} / \mathcal{E}_{0}$ such that the associated graded is a projective $R$-module and then one can take its top exterior power. This idea leads to a line bundle on $\widetilde{\mathrm{Gr}}_{N}$. Let us formulate it more generally for $\widetilde{\mathrm{Gr}}_{\mu_{\bullet}}$. Indeed, given a quasi-isogenies $\mathcal{E}_{i} \stackrel{\beta_{i}}{\rightarrow} \mathcal{E}_{i-1}$ with $\operatorname{Inv}\left(\beta_{i}\right) \in\left\{\omega_{1}, \ldots, \omega_{n}, \omega_{1}^{*}, \ldots, \omega_{n}^{*}\right\}$, we can define the line bundle

$$
\mathcal{L}_{i}=\left\{\begin{array}{cc}
\wedge^{j} \mathcal{E}_{i-1} / \mathcal{E}_{i} & \mu_{i}=\omega_{j}, \\
\wedge^{j} \mathcal{E}_{i} / \mathcal{E}_{i-1} & \mu_{i}=\omega_{j}^{*}
\end{array}\right.
$$

As $\operatorname{Gr}_{\mu}$ classifies a chain of quasi isogenies $\mathcal{E}_{n} \rightarrow \mathcal{E}_{n-1} \rightarrow \cdots \rightarrow \mathcal{E}_{0}$ with each quasi-isogeny of the above form. Then there are the line bundles $\mathcal{L}_{i}$, and we set

$$
\tilde{\mathcal{L}}_{\text {det }}=\bigotimes_{i} \mathcal{L}_{i}
$$

We form the following conjecture.

Conjecture I. There is a unique line bundle $\mathcal{L}_{\text {det }}$ on $\overline{\mathrm{Gr}}_{N}$ such that its pullback along $\widetilde{\mathrm{Gr}}_{N} \rightarrow \overline{\mathrm{Gr}}_{N}$ is $\tilde{\mathcal{L}}_{\text {det }}$ in B.1.1.

Conjecture II. A model of the line bundle $\mathcal{L}_{\text {det }}$ (in the sense as Lemma A.21) gives an embedding of a model of $\overline{\mathrm{Gr}}_{N}$ into some projective space.

We do not know how to prove Conjecture I at the moment. An evidence of Conjecture I is the following (we ignore the Tate twist).

Proposition B.1. The map $\mathrm{H}^{*}\left(\left(\overline{\mathrm{Gr}}_{N}\right)_{\bar{k}}, \overline{\mathbb{Q}}_{\ell}\right) \rightarrow \mathrm{H}^{*}\left(\left(\widetilde{\mathrm{Gr}}_{N}\right)_{\bar{k}}, \overline{\mathbb{Q}}_{\ell}\right)$ is injective, and there exists $c \in \mathrm{H}^{2}\left(\overline{\mathrm{Gr}}_{N}, \overline{\mathbb{Q}}_{\ell}\right)$, whose image in $\mathrm{H}^{2}\left(\widetilde{\mathrm{Gr}}_{N}, \overline{\mathbb{Q}}_{\ell}\right)$ is the Chern class $c\left(\tilde{\mathcal{L}}_{\mathrm{det}}\right)$.

We can give some reduction of Conjecture I. See Conjecture IV. First, we need

Proposition B.2. If $\Gamma\left(\widetilde{\mathrm{Gr}}_{\mu_{\bullet}}, \tilde{\mathcal{L}}_{\mathrm{det}}\right)$ is base point free, i.e. for every closed point $x \in \widetilde{\mathrm{Gr}}_{\mu_{\bullet}}$, there exists a section $s \in \Gamma\left(\widetilde{\mathrm{Gr}}_{\mu_{\bullet}}, \tilde{\mathcal{L}}_{\mathrm{det}}\right)$, such that $s(x) \neq 0$, then Conjecture I holds.

The proof of this proposition is based on the following two statements.

Lemma B.3. The algebraic space $\overline{\mathrm{Gr}}_{N}$ is of dimension $N(n-1)$, and the codimension of $\overline{\mathrm{Gr}}_{N}-\mathrm{Gr}_{N}$ is at least two.

This is a consequence of Lemma 1.18

Lemma B.4. The affine scheme $V_{N, h}^{\prime}$ defined via (1.3.1) is normal and is a locally complete intersection.

Note that assuming Conjecture I, Conjecture II holds if the space of global sections of $\mathcal{L}_{\text {det }}$ separate points. I.e. for every two different points $x, y \in \overline{\mathrm{Gr}}_{N}$, there exists $s \in \Gamma\left(\overline{\mathrm{Gr}}_{\mu_{\bullet}}, \mathcal{L}_{\text {det }}\right)$ such that $s(x)=0$ and $s(y) \neq 0$. We see in Remark 1.16, and in particular in the sequel $\$$ B.3 that in some cases when $\mu$ are very small, $\mathrm{Gr}_{\leq \mu}$ is the perfection of some projective variety. 
Remark B.5. More generally, given a perfect $\operatorname{ring} R$, one can define the following category $\mathcal{C}_{R}$ of triples $\left(\mathcal{E}_{1}, \mathcal{E}_{2}, \beta\right)$, where $\mathcal{E}_{1}$ and $\mathcal{E}_{2}$ are two finite projective $W(R)$-modules, and $\beta$ : $\mathcal{E}_{1}[1 / p] \rightarrow \mathcal{E}_{2}[1 / p]$ is an isomorphism. This is an exact category. It is an interesting question to know whether one can relate the algebraic K-theory of $\mathcal{C}_{R}$ to the K-theory of $R$.

B.2. Deperfection. Note that the perfect scheme $\operatorname{Gr}_{\mu}=L^{+} G / L^{+} G \cap \varpi^{\mu} L^{+} G \varpi^{-\mu}$ has a canonical model $\mathrm{Gr}_{\mu}^{\prime}$. Namely, we have the pro-algebraic group $L_{p}^{+} G$, which induces a canonical model of $L^{+} G \cap \varpi^{\mu} L^{+} G \varpi^{-\mu}$ as a reduced closed subgroup of $L_{p}^{+} G$, denote by $\Gamma_{0}\left(\varpi^{\mu}\right)$. Then $\mathrm{Gr}_{\mu}^{\prime}=L_{p}^{+} G / \Gamma_{0}\left(\varpi^{\mu}\right)$ is a smooth quasi-projective variety over $k$. As $\operatorname{Gr}_{\mu}$ is open dense in $\mathrm{Gr}_{\leq \mu}$, the construction of Lemma A.14 gives rise to a model $\mathrm{Gr}_{\leq \mu}^{\prime}$ of $\mathrm{Gr}_{\leq \mu}$, which is a proper algebraic space over $k$.

Conjecture III. The proper algebraic space $\mathrm{Gr}_{\leq \mu}^{\prime}$ is normal and Cohen-Macaulay.

An evidence of this conjecture is Lemma B.4

Remark B.6. If $\lambda<\mu$, there is the closed embedding $\mathrm{Gr}_{\leq \lambda} \subset \mathrm{Gr}_{\leq \mu}$, which by Proposition A.17, is induced from some $\mathrm{Gr}_{\leq \lambda}^{\prime} \rightarrow \mathrm{Gr}_{\leq \mu}^{\prime}$. However, this latter map is not a closed embedding and therefore we do not have a deperfection of the whole affine Grassmannian.

A natural question is whether $\mathrm{Gr}_{\leq \mu}^{\prime}$ has a natural moduli interpretation. We have no idea how to answer this question. The following discussion provides some hints that this might be an interesting question.

We consider $G=\mathrm{GL}_{n}$. As mentioned above, we do not know a moduli interpretation of $\overline{\mathrm{Gr}}_{N}^{\prime}:=\mathrm{Gr}_{\leq N \omega_{1}}^{\prime}$. On the other hand, recall that there is the "Demazure resolution" $\widetilde{\operatorname{Gr}}_{N} \rightarrow \overline{\mathrm{Gr}}_{N}$ and as in Lemma 1.18, $\operatorname{Gr}_{N} \subset \widetilde{\mathrm{Gr}}_{N}$ is open dense, so by the construction of Lemma A.14 we also have a canonical model $\widetilde{\mathrm{Gr}}_{N}^{\prime}$.

We do have a moduli interpretation of $\widetilde{\mathrm{Gr}}_{N}$, as suggested by L. Xiao. For simplicity, we assume that $k=\overline{\mathbb{F}}_{p}$. Fix $h \geq N$. Let $E / \mathbb{Q}_{p}$ be an unramified degree $2 h$ extension, with ring of integers $\mathcal{O}_{E}=\mathbb{Z}_{p^{2 h}}$. We fix an embedding $\tau_{0}: E \rightarrow \overline{\mathbb{Q}}_{p}$, and let $\tau_{i}=\sigma^{i} \tau_{0}$, where $\sigma: \overline{\mathbb{Q}}_{p} \rightarrow \overline{\mathbb{Q}}_{p}$ is (a lift of) the Frobenius automorphism. Then $\tau_{i+2 h}=\tau_{i}$.

Let $\mathbb{X}_{0}$ be a $p$-divisible group of height $2 h n$ and of dimension $h n$ over $\overline{\mathbb{F}}_{p}$, with an action $\iota: \mathcal{O}_{E} \rightarrow \operatorname{End\mathbb {X}_{0}}$. We assume that the signature of $\left(\mathbb{X}_{0}, \iota\right)$ is $(0, \ldots, 0, n, \ldots, n)$. I.e. $\operatorname{rk}\left(\operatorname{Lie} \mathbb{X}_{0}\right)_{\tau_{i}}=0$ for $i=0, \ldots, h-1$, where $\left(\operatorname{Lie} \mathbb{X}_{0}\right)_{\tau_{i}}=\operatorname{Lie} \mathbb{X}_{0} \otimes_{\mathcal{O}_{E}, \tau_{i}} \overline{\mathbb{F}}_{p}$.

We define a space $M_{N, h} \in \mathrm{Sp}_{\bar{F}_{p}}$ as follows. For an $\overline{\mathbb{F}}_{p}$-algebra $R$, the set $M_{N, h}(R)$ classify chains of isogenies of $p$-divisible groups with $\mathcal{O}_{E}$-actions on $R$,

$$
\left(\mathbb{X}_{0}\right)_{R} \stackrel{\phi_{1}}{\rightarrow} \mathbb{X}_{1} \rightarrow \cdots \stackrel{\phi_{N}}{\rightarrow} \mathbb{X}_{N}
$$

satisfying

(1) $\left(\mathbb{X}_{i}, \iota\right)$ has signature $(1, \ldots, 1,0 \ldots, 0, n, \ldots, n, n-1 \ldots, n-1)$, where the first $i$ positions are $n-1 \mathrm{~s}$ and the last $i$ positions are $1 \mathrm{~s}$;

(2) $\operatorname{deg} \phi_{i}=p^{2 i-1}$ for $i=1, \ldots, N$;

(3) $\left(d \phi_{i}\right)_{j}:\left(\operatorname{Lie} \mathbb{X}_{i-1}\right)_{\tau_{j}} \rightarrow\left(\operatorname{Lie} \mathbb{X}_{i}\right)_{\tau_{j}}$ is the zero map for $j=0, \ldots, h-1$; and

(4) $\left(d \phi_{i}^{*}\right)_{j}:\left(\operatorname{Lie} \mathbb{X}_{i}^{*}\right)_{\tau_{j}} \rightarrow\left(\operatorname{Lie} \mathbb{X}_{i-1}^{*}\right)_{\tau_{j}}$ is the zero map for $j=h, \ldots, 2 h-1$.

Note that the first two conditions imply that $\operatorname{ker} \phi_{i} \subset \mathbb{X}_{i-1}[p]$.

The first two conditions define a moduli scheme closely related to Rapoport-Zink spaces. However, it is not irreducible in general (not even equidimensional) and the last two conditions cut out $M_{N, h}$ inside it as an irreducible component. More precise, by induction we have

Lemma B.7. The space $M_{i+1, h}$ is a $\mathbb{P}^{n-1}$-bundle over $M_{i, h}$, for $i=1, \ldots, h$. Therefore, $M_{N, h}$ is represented by a smooth projective variety. 
Indeed, let $\mathbb{X}_{i}^{\text {univ }}$ denote the universal $p$-divisible group on $M_{i, h}$ appearing at the end of the chain. Let $M\left(\mathbb{X}_{i}^{\text {univ }}\right)^{*}$ denote the dual of the Lie algebra of the universal extension of $\mathbb{X}_{i}^{\text {univ }}$ by vector groups. Let $M\left(\mathbb{X}_{i}^{\text {univ }}\right)_{\tau_{j}}^{*}=M\left(\mathbb{X}_{i}^{\text {univ }}\right)^{*} \otimes_{\mathcal{O}_{E}, \tau_{j}} \overline{\mathbb{F}}_{p}$. Then we have a rank $n$ vector bundle $E_{i}=M\left(\mathbb{X}_{i}^{\text {univ }}\right)_{\tau_{0}}^{*}$ on $M_{i, h}$ and $M_{i+1, h}=\mathbb{P}\left(E_{i}\right)$.

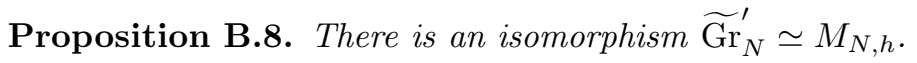

Indeed, choosing a trivialization $\mathbb{D}\left(\mathbb{X}_{0}\right) \simeq W(k)^{n}=\mathcal{E}_{0}$, and using the argument as in Proposition 3.12, one shows that $M_{N, h}^{p^{-\infty}} \simeq \widetilde{\mathrm{Gr}}_{N}$ given by sending $\mathbb{X}_{0} \rightarrow \cdots \rightarrow \mathbb{X}_{N}$ to $\mathbb{D}\left(\mathbb{X}_{N}\right)_{\tau_{0}} \rightarrow \cdots \rightarrow \mathbb{D}\left(\mathbb{X}_{0}\right)_{\tau_{0}}$. On the other hand, there exists an open subscheme $\stackrel{\circ}{M}, h_{N} \subset$ $M_{N, h}$ parameterizing those chains such that the kernel of the map $\phi_{N} \cdots \phi_{1}: \mathbb{X}_{0} \rightarrow \mathbb{X}_{N}$ is not contained in $\mathbb{X}_{0}\left[p^{N-1}\right]$ and one can show that $\stackrel{\circ}{M}, h_{N} \simeq \mathrm{Gr}_{N}^{\prime}$, compatible with $\widetilde{G r}_{N} \simeq$ $M_{N, h}^{p^{-\infty}}$. Then by Remark A.16, we have a projective birational universal homeomorphism $\widetilde{\mathrm{Gr}}_{N}^{\prime} \rightarrow M_{N, h}$. As $M_{N, h}$ is a smooth projective variety, we have $\widetilde{\mathrm{Gr}}_{N}^{\prime} \simeq M_{N, h}$. Details are left to readers.

There is a line bundle on $M_{N, h}$ given by

$$
\tilde{\mathcal{L}}_{\mathrm{det}}^{\prime}:=\bigotimes_{i=1}^{N} \omega_{\mathbb{X}_{N}, \tau_{-i}}^{-p^{i}}
$$

where $\omega_{\mathbb{X}_{N}, \tau_{j}}=\wedge^{\text {top }}\left(\operatorname{Lie} \mathbb{X}_{N}\right)_{\tau_{j}}^{*}$.

Lemma B.9. Under the map $\widetilde{\mathrm{Gr}}_{N} \simeq M_{N, h}^{p^{-\infty}} \stackrel{\varepsilon}{\rightarrow} M_{N, h}, \tilde{\mathcal{L}}_{\mathrm{det}} \simeq \varepsilon^{*} \tilde{\mathcal{L}}_{\text {det }}^{\prime}$.

Therefore, in view of Proposition B.2. Conjecture I would be a consequence of

Conjecture IV. The line bundle $\tilde{\mathcal{L}}_{\text {det }}^{\prime}$ on $M_{N, h}$ is semi-ample.

B.3. Example: $\widetilde{\mathrm{Gr}}_{2} \rightarrow \overline{\mathrm{Gr}}_{2}$. We assume that $p>2$ so that the Teichmüller lifting of -1 is -1 . The purpose here is to illustrate our the construction of $\$ 1.3$ in the simplest but non-trivial case: $G=\mathrm{GL}_{2}$, and $N=2$. So we study $\widetilde{\mathrm{Gr}}_{2} \rightarrow \overline{\mathrm{Gr}}_{2}$. We hope to convince the readers that the bizzarre-looking construction is indeed reasonable. In particular, we show that $\overline{\mathrm{Gr}}_{2}$ is the perfection $\overline{\mathrm{Gr}}_{2}^{b}$, and a model of $\widetilde{\mathrm{Gr}}_{2} \rightarrow \overline{\mathrm{Gr}}_{2}$ can be regarded as a resolution of the singularity of $\overline{\mathrm{Gr}}_{2}$. We follow the notation of $\$ 1.3$.

First, it is easy to understand $\widetilde{\mathrm{Gr}}_{2}$. Indeed, $\widetilde{\mathrm{Gr}}_{1}=\mathbb{P}^{1, p^{-\infty}}$, over which there is $\mathcal{E} / p \rightarrow$ $\mathcal{O}_{\mathbb{P 1} 1, p^{-\infty}}^{2} \rightarrow \mathcal{O}_{\mathbb{P}^{1, p},-\infty}(1)$. Then $\widetilde{G r}_{2}=\mathbb{P}^{p^{-\infty}}(\mathcal{E} / p)$. From (1.4.1), we know that $\mathcal{E} / p$ fits into the following exact sequence

$$
0 \rightarrow \mathcal{O}(1) \rightarrow \mathcal{E} / p \rightarrow \mathcal{O}(-1) \rightarrow 0 .
$$

Then $\mathcal{E} / p \simeq \mathcal{O}(1) \oplus \mathcal{O}(-1)$ (but this isomorphism is non-canonical). Therefore, $\widetilde{\mathrm{Gr}}_{2}$ is isomorphic to $\mathbb{P}^{p^{-\infty}}(\mathcal{O}(1) \oplus \mathcal{O}(-1))$.

Next we consider $\overline{\mathrm{Gr}}_{2}$. According to the previous subsection,

$$
\overline{\mathrm{Gr}}_{2}=\overline{\mathrm{Gr}}_{2,3} / L^{3} \mathrm{GL}_{2} \text {. }
$$

Consider the scheme $U=\operatorname{Spec} R$ where $R=k[x, y, z] / x^{2}-y z$. There is a natural map $U^{p^{-\infty}} \rightarrow \overline{\mathrm{Gr}}_{2}$ given by

$$
(x, y, z) \mapsto\left(\mathcal{E}_{0}, A\right) \quad \text { where } A=\left(\begin{array}{cc}
p+[x] & -[y] \\
{[z]} & p-[x]
\end{array}\right) \in \mathrm{GL}_{2}\left(W\left(R^{p^{-\infty}}\right)[1 / p]\right) .
$$

Lemma B.10. This is an open embedding. 
We write $G$ for $L^{3} \mathrm{GL}_{2}$ for simplicity. We lift $U^{p^{-\infty}} \rightarrow \overline{\mathrm{Gr}}_{2}$ to $U^{p^{-\infty}} \rightarrow \overline{\mathrm{Gr}}_{2,3}$ as $A \mapsto$ $\left(\mathcal{E}_{0}, A\right.$, id). Then we need to show that the action map $U^{p^{-\infty}} \times G \rightarrow \overline{\mathrm{Gr}}_{2,3}$ is an open embedding.

This will follow from the following lemma whose proof is based some linear algebra calculation.

Lemma B.11. The subfunctor $W \subset V_{2,3}$, such that for $X \in W$, there exists $g \in G$ and $A=\left(\begin{array}{cc}p+[x] & -[y] \\ {[z]} & p-[x]\end{array}\right)$ with $x^{2}=y z$ satisfying $X=A g$, is an open subscheme. In addition, for $X \in W$, and write $X=A g$, then such $A$ is unique.

Proof. Let $X=\left(\begin{array}{ll}a & b \\ c & d\end{array}\right) \in V_{2,3}$, and $X^{*}=\left(\begin{array}{cc}d & -b \\ -c & a\end{array}\right)$ be its adjugate matrix. We expand $a=\left[a_{0}\right]+p\left[a_{1}\right]+p^{2}\left[a_{2}\right]$, and similarly $b, c, d$. The condition that $\operatorname{det} X$ is divisible by $p^{2}$ is translated into the following equations

$$
a_{0} d_{0}=b_{0} c_{0}, \quad\left(\begin{array}{ll}
a_{1} & b_{1} \\
c_{1} & d_{1}
\end{array}\right)\left(\begin{array}{cc}
d_{0} & -b_{0} \\
-c_{0} & a_{0}
\end{array}\right)+\left(\begin{array}{ll}
a_{0} & b_{0} \\
c_{0} & d_{0}
\end{array}\right)\left(\begin{array}{cc}
d_{1} & -b_{1} \\
-c_{1} & a_{1}
\end{array}\right)=0 .
$$

I.e. $V_{2,3}$ is represented by an open subscheme of the affine scheme defined by the equations (B.3.1).

Now if there exists $A, g$ such that $X=A g$. Left multiplying the equation by $X^{*}$, we see that

$$
p^{2}[\lambda] g^{-1}=\left(\begin{array}{cc}
d & -b \\
-c & a
\end{array}\right)\left(\begin{array}{cc}
p+[x] & -[y] \\
{[z]} & p-[x]
\end{array}\right) .
$$

The fact that the r.h.s. is divisible by $p^{2}$, is translated into the following two matrix equations in $R$.

$$
\left(\begin{array}{cc}
d_{0} & -b_{0} \\
-c_{0} & a_{0}
\end{array}\right)\left(\begin{array}{cc}
x & -y \\
z & -x
\end{array}\right)=0, \quad\left(\begin{array}{cc}
d_{1} & -b_{1} \\
-c_{1} & a_{1}
\end{array}\right)\left(\begin{array}{cc}
x & -y \\
z & -x
\end{array}\right)=-\left(\begin{array}{cc}
d_{0} & -b_{0} \\
-c_{0} & a_{0}
\end{array}\right) .
$$

In other words, a necessary condition for the existence of such $(A, g)$ is that (B.3.2) has solutions $x, y, z$.

Note that on the open subscheme $\tilde{W}$ of $V_{2,3}$ defined by $a_{1} d_{1}-b_{1} c_{1} \in R^{*}$, we can solve $x, y, z$ uniquely as

$$
\left(\begin{array}{cc}
x & -y \\
z & -x
\end{array}\right)=\frac{1}{b_{1} c_{1}-a_{1} d_{1}}\left(\begin{array}{cc}
a_{1} & b_{1} \\
c_{1} & d_{1}
\end{array}\right)\left(\begin{array}{cc}
d_{0} & -b_{0} \\
-c_{0} & a_{0}
\end{array}\right)
$$

One can use (B.3.1) to check that the above definition of $(x, y, z)$ is valid and such defined $(x, y, z)$ satisfy (B.3.2).

Now, let $X \in \tilde{W}$ and $A=\left(\begin{array}{cc}p+[x] & -[y] \\ {[z]} & p-[x]\end{array}\right)$, where $(x, y, z)$ is given by (B.3.3). Using the lifting $V_{2,3} \rightarrow L^{+} V_{2}$ as in Remark 1.12, we regard $X$ and $A$ as element in $L^{+} V_{2} \subset L \mathrm{GL}_{2}$, denoted by $\tilde{X}$ and $\tilde{A}$. Then

$$
\tilde{g}:=\tilde{X} \tilde{A}^{-1}=p^{-2} \tilde{X} \tilde{A}^{*} \in L \mathrm{GL}_{2}
$$

has determinant in $\mathcal{O}^{*}$. But as B.3.2 holds, the entries $\tilde{g}$ are in fact in $\mathcal{O}$. Therefore, $\tilde{g} \in L^{+} \mathrm{GL}$. Let $g=\left(\tilde{g} \bmod p^{3}\right)$, then $X=A g$. In other words, for $X \in \tilde{W}, X=A g$ has solutions $(A, g)$. In addition, such $A$ is unique. Now, let $W=\tilde{W} G$ be the minimal $G$ invariant open subset of $V_{2,3}$ containing $\tilde{W}$. Then for every $X \in W, X=A g$ has solutions and $A$ is unique. On the other hand, if $X=A g$, as $A \in \tilde{W}, X \in W$. Therefore, $W$ is exactly the subfunctor of $V_{2,3}$ such that $X=A g$ has a solution. The lemma follows.

Now let $V$ be the preimage of $W$ under $\overline{\mathrm{Gr}}_{2,3} \rightarrow V_{2,3}$. Then $U^{p^{-\infty}} \times G \rightarrow V$ is an isomorphism. In fact, the uniqueness of $A$ as in the lemma implies the injectivity of the map 
for any $R$-points. On the other hand, the definition of $W$ together with an argument as in Lemma 1.11 implies the surjectivity. Therefore the lemma holds.

In this case, one can show that the invertible sheaf $\tilde{\mathcal{L}}_{\text {det }}$ on $\widetilde{\mathrm{Gr}}_{2}$ descends to an invertible sheaf $\mathcal{L}_{\text {det }}$ on $\overline{\mathrm{Gr}}_{2}$. Namely, Let $j: \mathrm{Gr}_{2} \rightarrow \overline{\mathrm{Gr}}_{2}$ be the open inclusion. We can restrict $\tilde{\mathcal{L}}_{\text {det }}$ to $\mathrm{Gr}_{2} \subset \widetilde{\mathrm{Gr}}_{2}$. Then $\mathcal{L}_{\text {det }}=j_{*}\left(\left.\mathcal{L}_{\mathrm{det}}\right|_{\mathrm{Gr}_{2}}\right)$.

\section{REFERENCES}

[BL] A. Beauville, Y. Laszlo, Conformal Blocks and Generalized Theta Functions, Commun. Math. Phys. 164,385-419(1994).

[BD] A. Beilinson, V. Drinfeld, Quantization of Hitchin's integrable system and Hecke eigensheaves, Preprint, available at www.math.uchicago.edu/ mitya/langlands.

[CKV] M. Chen, M. Kisin, E. Viehmann, Connected components of affine Deligne-Lusztig varieties in mixed characteristic, arXiv:1307.3845.

[Co] B. Conrad, The Keel-Mori theorem via stacks, available at http://math.stanford.edu/ conrad/

[CLO] B. Conrad, M. Lieblich, M. Olsson, Nagata compactification for algebraic spaces, Journal of the Institute of Mathematics of Jussieu, Volume 11, Issue 04, October 2012, pp 747-814

[EGAIV] A. Grothendieck; J. Dieudonné, Éléments de géométrie algébrique: IV. Étude locale des schémas et des morphismes de schémas, Troisième partie, Publications Mathématiques de l'IHÉS 28, 5-255 (1966).

[EGAIV] A. Grothendieck; J. Dieudonné, Éléments de géométrie algébrique: IV. Étude locale des schémas et des morphismes de schémas, Quatrième partie, Publications Mathématiques de l'IHÉS 32, 5-361 (1967).

[Ga] D. Gaitsgory, Construction of central elements in the affine Hecke algebra via nearby cycles, Invent. Math. 144 (2001), no. 2, 253-280.

[Gi] V. Ginzburg, Perverse sheaves on a loop group and Langlands' duality, arXiv:math/9511007

[GHKR] U. Görtz, T. Haines, R. Kottwitz, Dimensions of some affine Deligne-Lusztig varieties, Ann. Scient. Ecole Norm. Sup., (4) 39 (2006), 467-511.

[Grb] M. J. Greenberg, Schemata Over Local Rings, Annals of Mathematics 73 (1961), no. 3, 624-648.

[Ha] W. Haboush, Infinite dimensional algebraic geometry: algebraic structures on p-adic groups and their homogeneous spaces, Tohoku Math. J. (2) 57 (2005), no. 1, 65-117.

[Ham1] P. Hamacher, The geometry of Newton strata in the reduction modulo $p$ of Shimura varieties of PEL type, arXiv:1312.0490

[Ham2] P. Hamacher, The dimension of affine Deligne-Lusztig varieties in the affine Grassmannian of unramified groups, arXiv:1312.0486

[Ka] N. Katz, Slope filtrations for F-crystals, Astèrisque 63 (1979), 113163.

[KL1] D. Kazhdan, G. Lusztig, Representations of Coxeter groups and Hecke algebras, Invent. Math. 53 (1979), 165184.

[KL2] D. Kazhdan, G. Lusztig, Schubert varieties and Poincaré duality, In Geometry of the Laplace operator, Proc. Sympos. Pure Math., XXXVI, Amer. Math. Soc., Providence, R.I., 1980.

[KM] S. Keel, S. Mori, Quotients by groupoids, Ann. of Math. (2) 145 (1997), 193-213.

[Kim] W. Kim, Rapoport-Zink spaces of Hodge type, arXiv:1308.5537

[Kn] D. Knuston, Algebraic spaces, Lecture Notes in Math. 203, Springer-Verlag, Berlin-New York, 1971.

[Ko] R. E. Kottwitz, Isocrystals with additional structure. II, Comp. Math. 109 (1997), 255339.

[Kr] M. Kreidl, On p-adic lattices and Grassmannians, arXiv:1010.0349

[Laf] V. Lafforgue, Chtoucas pour les groupes réductifs et paramétrisation de Langlands globale, arXiv:1209.5352

[La] E. Lau, Smoothness of the truncated display functor, J. Amer. Math. Soc. 26 (2013), 129-165.

[LMB] G. Laumon, L. Moret-Bailly, Champs algébriques, Ergebnisse der Mathematik und ihrer Grenzgebiete (3 Folge, A Series of Modern Surveys in Mathematics), 39, Springer-Verlag, Berlin, 2000.

[Lu1] G. Lusztig, Singularities, character formulas, and a q-analog of weight multiplicities, In Analysis and topology on singular spaces, II, III (Luminy, 1981), 208-229, Astérisque, 101-102, Soc. Math. France, Paris, 1983.

[Lu2] G. Lusztig, A bar operator for involutions in a Coxeter group, Bull. Inst. Math. Acad. Sinica (N.S.) $7(2012), 355-404$.

[LV] G. Lusztig, D. Vogan, Hecke algebras and involutions in Weyl groups, Bull. Inst. Math. Acad. Sinica (N.S.) 7(2012), 323-354.

[LY] G. Lusztig, Z. Yun, A ( $-q)$-analog of weight multiplicities, Journal of the Ramanujan Math. Soc., 28A (Special Issue-2013) 311-340.

[MV] I. Mirkovic̀, K. Vilonen: Geometric Langlands duality and representations of algebraic groups over commutative rings, Ann. of Math. (2) 166 (2007), no. 1, 95-143. 
[NP] B. C. Ngô, P. Polo, Résolutions de Demazure affines et formule de Casselman-Shalika géométrique, J. Algebraic Geom. 10 (2001), no. 3., 515-547.

[PR] G. Pappas, M. Rapoport, Twisted loop groups and their affine flag varieties, Adv. in Math., 219 (2008) 118-198.

[R] M. Rapoport, A guide to the reduction modulo $p$ of Shimura varieties, Astérisque 298 (2005) 271-318.

[RZ] M. Rapoport, T. Zink, Period spaces for p-divisible groups, Ann. Math. Studies 141, Princeton University Press, 1996.

[RG] L. Gruson, M. Raynaud, Critères de platitude et de projectivité, Inventiones Math. 13, 1971, pp. 1-89.

[Ri] T. Richarz, A new approach to the geometric Satake equivalence, Documenta Mathematica 19 (2014) 209-246.

[SGA3] A. Grothendieck, M. Demazure, Propriétés Générales des Schémas en Groupes, Lecture Notes in Mathematics 151, Springer-Verlag, 1970.

[SGA4] A. Grothendieck, M. Artin,. Théorie des Topos et Cohomologie Etale des Schémas, Lecture Notes in Mathematics 269, Springer-Verlag, 1972.

[Se] J. P. Serre, Groupes proalgébriques, Publ. math. de l'I.H.É.S., tom 7 (1960), p. 5-67.

[St] Stack project authors Stack project, http://stacks.math.columbia.edu, 2014.

[V1] E. Viehmann, The dimension of some affine Deligne-Lusztig varieties, Ann. Sci. École Norm. Sup. (4) 39 (2006), no. 3, 513-526.

[V2] E. Viehmann, moduli of p-divisible groups, J. Algebraic Geom. 17 (2008), no. 2, 341-374.

[V3] E. Viehmann, The global structure of moduli spaces of polarized p-divisible groups, Doc. Math. 13 (2008), 825-852.

[XZ] L. Xiao, X. Zhu, Cycles on Shimura varieties via geometric Satake, in preparation.

E-mail address: xinwenz@math.northwestern.edu 